\title{
Construction of highly accurate models of rotating neutron stars - comparison of three different numerical schemes
}

\author{
T. Nozawa ${ }^{1}$, N. Stergioulas ${ }^{2, \star}$, E. Gourgoulhon ${ }^{3}$, and Y. Eriguchi ${ }^{1}$ \\ 1 Department of Earth Science and Astronomy, Graduate School of Arts and Sciences, University of Tokyo, Komaba, Meguro, \\ Tokyo 153, Japan \\ 2 Department of Physics, University of Wisconsin-Milwaukee, PO box 413, Milwaukee, WI 53201, U.S.A. \\ 3 Département d'Astrophysique Relativiste et de Cosmologie, UPR 176 du CNRS, Observatoire de Paris, F-92195 Meudon \\ Cedex, France
}

Received April 15; accepted May 6, 1998

\begin{abstract}
We conduct a direct comparison of three different representative numerical codes for constructing models of rapidly rotating neutron stars in general relativity. Our aim is to evaluate the accuracy of the codes and to investigate how the accuracy is affected by the choice of interpolation, domain of integration and equation of state. In all three codes, the same physical parameters, equations of state and interpolation method are used. We construct 25 selected models for polytropic equations of state and 22 models with realistic neutron star matter equations of state. The three codes agree well with each other (typical agreement is better than $0.1 \%$ to $0.01 \%$ ) for most models, except for the extreme assumption of uniform density stars. We conclude that the codes can be used for the construction of highly accurate initial data configurations for polytropes of index $N>0.5$ (which typically correspond to realistic neutron stars), when the domain of integration includes all space and for realistic equations with no phase transitions. With the exception of the uniform density case, the obtained values of physical parameters for the models considered in this paper can be regarded as "standard" and we display them in detail for all models.
\end{abstract}

Key words: stars: neutron — stars: rotation — pulsars: general — relativity — methods: numerical

\section{Introduction}

The physical state of the neutron star matter has not been fully understood yet because it is very difficult to inves-

Send offprint requests to: E. Gourgoulhon

* Present address: Max Planck Institute for Gravitational Physics, The Albert Einstein Institute, D-14473 Potsdam, Germany. tigate particle interactions beyond nuclear matter density $\left(\varepsilon_{\mathrm{N}} / c^{2} \sim 210^{14} \mathrm{~g} \mathrm{~cm}^{-3}\right)$ either from nuclear experiments or from nuclear theories, (here $\varepsilon_{N}$ is the energy density of the nuclear matter and $c$ is the velocity of light). Given this situation, one promising approach to explore the behavior of very high density matter is to make use of the macroscopic quantities of neutron stars. In particular, the mass and the rotational period of neutron stars depend crucially on the softness of the equation of state (EOS) at very high densities (see e.g. Friedman et al. 1984, 1986, 1989), thus, observational constrains, matched with theoretical models, may help in reconstructing the equation of state of very high density matter.

Given a particular equation of state, the mass of neutron stars varies with central energy density and always reaches a maximum. This implies that if the maximum mass of neutron star models constructed with a certain equation of state is smaller than the mass of observed neutron stars, that equation of state must be discarded. Currently, the largest accurately measured mass of slowly rotating neutron stars is $M_{\mathrm{BP}}=1.44 M_{\odot}$, where $M_{\mathrm{BP}}$ is the mass of one of the components of the binary pulsar PSR1913+16 (Taylor \& Weisberg 1989) and $M_{\odot}$ is one solar mass. Individual masses of neutron stars have also been estimated in six other binary pulsars (Thorsett et al. 1993; Wolszczan 1997), as well as in six X-ray binaries (van Kerkwijk et al. 1995) but the accuracy is not as good as in PSR1913+16. Thus, equations of state which give larger masses than $M_{\mathrm{BP}}$ for slowly rotating stars, can be valid as candidates for the real equation of state at very high densities. Since the maximum mass of neutron stars is smaller for more compressible (soft) equations of state than for less compressible (stiff) equations of state, the true equation of state at high densities cannot be extremely soft. 
On the other hand, stiff equations of state can be limited by considering the neutron star with the shortest rotational period, i.e. the most rapidly rotating pulsar. There exists a lower limit on the rotational period for each equation of state, because if the centrifugal force exceeds the self-gravity at the equatorial surface, no equilibrium states are allowed. The lower limit of the rotational period depends on the softness of the equation of state - the radius of neutron stars with softer equations of state is smaller, which allows for higher rotation rates. Thus, if very rapidly rotating neutron stars should be found, we could exclude most stiff equations of state. At the moment, the shortest period of observed pulsars is $1.56 \mathrm{~ms}$, of PSR1937+21. Consequently, equations of state for which the shortest rotational period is larger than this value, must be excluded as candidates for the real equation of state for neutron star matter.

The discussions above require us to make use of highly accurate schemes for constructing rotating neutron star models, in order to compute precise theoretical values of masses and rotational periods. Highly accurate relativistic equilibrium models are also needed as initial data for relativistic time-evolution codes (modeling of nonlinear pulsations, collapse and generation of gravitational waves). Recently, a number of groups have succeeded in constructing models of rapidly rotating neutron stars (Friedman et al. 1984, 1986, 1988, 1989; Eriguchi et al. 1994; Salgado et al. 1994a,b; Cook et al. 1994b; Stergioulas \& Friedman 1995 - for a recent review see Stergioulas 1998). However, the obtained models by those authors do not always agree with each other (see e.g. Friedman et al. 1989; Eriguchi et al. 1994; Salgado et al. 1994a; Cook et al. 1994b; Stergioulas \& Friedman 1995). Although Stergioulas \& Friedman (1995) have determined the cause of the discrepancy between models in Friedman et al. (1989) and Eriguchi et al. (1994), (which was due to the use of a slightly different equation of state table), the reasons of smaller differences which remain, even after using exactly the same equation of state, have not been clarified yet. This is because numerical techniques used in the different codes, such as the choice of parameters defining the model, the interpolation method, the method of integrating the field equations, a.s.o. are not the same.

In this paper, three groups using their own codes (Komatsu et al. 1989a; Eriguchi et al. 1994; Salgado et al. 1994a; Stergioulas \& Friedman 1995) will decrease the differences between their results to a minimum possible, by tuning each code and using the same parameters, the same schemes of interpolation, the same equations of state, and so on. Since the basic schemes used by the three groups are different, it will be impossible to have exactly the same results and the relative differences between results are a measure of the accuracy of the codes. Models obtained with small relative differences between the three codes can be considered as "standard" models for each equation of state. Furthermore, this direct comparison allows us to investigate the effect that the choice of interpolation method, equation of state and domain of integration has on the accuracy of the codes.

\section{Construction of neutron star models}

If rapidly rotating neutron stars were nonaxisymmetric, they would emit gravitational waves in a very short time scale and settle down to axisymmetric configurations. Moreover, the gravity of typical neutron star is strong because

$\frac{2 G M_{\mathrm{ns}}}{c^{2} R_{\mathrm{ns}}} \sim 0.4$

where $G$ is the gravitational constant, and $M_{\mathrm{ns}} \sim 1.4 M_{\odot}$ and $R_{\mathrm{ns}} \sim 10 \mathrm{~km}$ are the mass and the radius of a typical neutron star. Therefore, we need to solve for rotating and axisymmetric configurations in the framework of general relativity.

For the matter and the spacetime we make the following assumptions:

1. The matter distribution and the spacetime are axisymmetric.

2. The matter and the spacetime are in a stationary state.

3. The matter has no meridional motions. The only motion of the matter is a circular one that is represented by the angular velocity.

4. The angular velocity $\Omega$ is constant, as seen by a distant observer at rest.

5. The matter can be described as a perfect fluid.

Under these assumptions, the metric can be expressed as follows (Papapetrou 1966; Carter 1969):

$$
\begin{aligned}
\mathrm{d} s^{2}= & -\mathrm{e}^{2 \nu} \mathrm{d} t^{2}+\mathrm{e}^{2 \alpha}\left(\mathrm{d} r^{2}+r^{2} \mathrm{~d} \theta^{2}\right) \\
& +\mathrm{e}^{2 \beta} r^{2} \sin ^{2} \theta(\mathrm{d} \varphi-\omega \mathrm{d} t)^{2} \\
= & -\mathrm{e}^{2 \nu} \mathrm{d} t^{2}+\mathrm{e}^{2 \zeta-2 \nu}\left(\mathrm{d} r^{2}+r^{2} \mathrm{~d} \theta^{2}\right) \\
& +B^{2} \mathrm{e}^{-2 \nu} r^{2} \sin ^{2} \theta(\mathrm{d} \varphi-\omega \mathrm{d} t)^{2} \\
= & -\mathrm{e}^{2 \nu} \mathrm{d} t^{2}+\mathrm{e}^{2 \mu}\left(\mathrm{d} r^{2}+r^{2} \mathrm{~d} \theta^{2}\right)+\mathrm{e}^{2 \psi}(\mathrm{d} \varphi-\omega \mathrm{d} t)^{2}
\end{aligned}
$$

where $t$ is the time coordinate and the polar coordinates $(r, \theta, \varphi)$ are used. The metric depends on four metric functions (or metric potentials) which are functions of $r$ and $\theta$ only. Different authors have used the set of functions $(\nu, \omega, \alpha, \beta),(\nu, \omega, \zeta, B)$ or $(\nu, \omega, \mu, \psi)$, which are related to each other through (2). In (2) and throughout the text we use gravitational units $(c=G=1)$, unless otherwise stated.

The energy momentum tensor $T^{\mathrm{ab}}$ is expressed as

$T^{\mathrm{ab}}=(\varepsilon+p) u^{\mathrm{a}} u^{\mathrm{b}}+p g^{\mathrm{ab}}$,

where $\varepsilon, p$ and $u^{\mathrm{a}}$ are the energy density, the pressure and the four-velocity, respectively. In the coordinate basis defined by (2), the components of the four-velocity are

$u^{\mathrm{a}}=\frac{\mathrm{e}^{-\nu}}{\sqrt{1-v^{2}}}(1,0,0, \Omega)$ 
where the proper velocity $v$ with respect to a local zero angular momentum observer is defined by

$v \equiv r \sin \theta \mathrm{e}^{\beta-\nu}(\Omega-\omega)$.

Using the metric and the energy momentum tensor mentioned above, we can write down the Einstein equations for the metric components. Although we omit detailed expressions for the Einstein equations here, one can easily derive them by straightforward calculations or consult the papers by Butterworth \& Ipser (1976), Komatsu et al. (1989a) and Bonazzola et al. (1993).

The equation of hydrostationary equilibrium can be derived from the equations of motion and takes the following form:

$\frac{1}{\varepsilon+p} \nabla p+\nabla \nu-\frac{1}{2} \nabla \ln \left(1-v^{2}\right)=0$.

This equation can be integrated, if we specify the equation of state which relates the energy density to the pressure. For a given EOS, a model is uniquely specified by fixing two parameters, such as the central energy density $\epsilon_{c}$ and the ratio of the polar to the equatorial coordinate radius, $r_{\mathrm{p}} / r_{\mathrm{e}}$. Then, four Einstein field equations and the equation of hydrostationary equilibrium must be solved with appropriate boundary conditions, to yield the four metric functions and the density distribution. The available codes for obtaining relativistic rotating neutron star models differ basically in the choice and method of integration of the four field equations and in the finite grid and finite difference scheme used for the integration.

\section{Codes}

We will compare three different codes, which follow the KEH scheme, developed by Komatsu et al. (1989a,b) for general relativistic polytropes with uniform and differential rotation and improved by Cook et al. (1992, 1994a,b) and the BGSM scheme due to Bonazzola et al. (1993).

Concerning the KEH scheme, for the present comparison, we will use the original $\mathrm{KEH}$ code, $\mathrm{KEH}(\mathrm{OR})$, and the KEH code by Stergioulas \& Friedman (1995), KEH(SF), which follows the Cook et al. (1992, 1994a,b) modification of the KEH scheme.

\subsection{A short description of each code}

In this section the three different numerical codes are briefly described. Details of these codes can be found in Komatsu et al. (1989a,b) and in Eriguchi et al. (1994) for the $\mathrm{KEH}(\mathrm{OR})$ code, in Stergioulas \& Friedman (1995) for the $\mathrm{KEH}(\mathrm{SF})$ code and in Bonazzola et al. (1993) for the BGSM code.

\subsubsection{The $\mathrm{KEH}(\mathrm{OR})$ code}

Komatsu et al. (1989a) have developed a new scheme for solving rapidly rotating relativistic stars. The Einstein equations for three metric potentials $\nu, \beta$ and $\omega$ are transformed into integral equations by using appropriate Green's functions for the elliptical type differential operators. In principle, one can choose Green's functions which decrease as $1 / r$ or more rapidly at large distances. Consequently boundary conditions at infinity, i.e. asymptotically flat conditions can be easily included in the integral equations. It is noted that in this integral representation the integrand contains the metric and the matter quantities such as the energy density and the pressure. The fourth metric $\alpha$ obeys a first order partial differential equation which can be easily integrated, if the other metric potentials are known. The domain of integration is truncated at a finite distance from the star (roughly twice the equatorial radius) and the metric potentials in the integrands are assumed to vanish at that finite distance (instead of at infinity).

The KEH scheme is the extended version of the selfconsistent-field (SCF) scheme which was developed for solving Newtonian rotating stars (Ostriker \& Mark 1968; Hachisu 1986) and applied to relativistic rotating stars by Bonazzola \& Schneider (1974) with a choice of metric functions different from that of the codes considered in this article. In the SCF method, the iteration proceeds as follows. If one assumes initial guesses for the matter quantities and the metric potentials, new (and better) values for $\nu, \beta$ and $\omega$ can be obtained using the integral representations for the metric potentials The fourth metric potential $\alpha$ can be easily solved as mentioned before. By using newly obtained metric potentials, a new density and a new pressure can be computed from the hydrostationary equilibrium Eq. (8). One needs to repeat the same procedure until the relative differences between the newly obtained quantities and the old ones become less than a certain small number, typically $10^{-5}$.

In the original $\mathrm{KEH}$ code, the ratio of the central pressure $\left(p_{\mathrm{c}}\right)$ to the central energy density $\left(\varepsilon_{\mathrm{c}}\right)$, $\kappa \equiv \frac{p_{\mathrm{c}}}{\varepsilon_{\mathrm{c}}}$

and the ratio of the polar radius $\left(r_{\mathrm{p}}\right)$ to the equatorial radius $\left(r_{\mathrm{e}}\right), r_{\mathrm{p}} / r_{\mathrm{e}}$, are chosen as two independent model parameters which specify the model uniquely. The $\mathrm{KEH}(\mathrm{OR})$ code used in this comparison differs from the code used in Komatsu et al. (1989a) only in one aspect, that is mentioned in the next section.

\subsubsection{The $\mathrm{KEH}(\mathrm{SF})$ code}

The KEH(SF) code (Stergioulas \& Friedman 1995) differs from the original KEH scheme in two ways. First, it follows Cook et al. (1992) in using a redefined radial variable $s \equiv \frac{r}{r+r_{\mathrm{e}}}$. 
where $r_{\mathrm{e}}$ is the value of the coordinate $r$ at the equator. By this transformation, the region $[0, \infty]$ in the $r$ coordinate is transformed to the region $[0,1]$ in the $s$ coordinate. Consequently, the domain of integration of the field equations does not have to be truncated at a finite distance from the star (as in the $\mathrm{KEH}(\mathrm{OR})$ code) but covers all space. With this choice, the boundary conditions can be applied accurately at infinity.

Second, Stergioulas \& Friedman found that the choice of coordinates in the original $\mathrm{KEH}$ scheme results in the metric potential $\alpha$ oscillating in the radial direction. The oscillation is especially pronounced inside the star and introduces an error of $1-2 \%$ in the mass, radius and other quantities. The problem was fixed by using a finite difference formula for the second order radial derivative that uses twice the grid-spacing. Although this formula is, in principle, of lower accuracy, the oscillations are damped completely, resulting in a more accurate stellar model.

The $\mathrm{KEH}(\mathrm{OR})$ code used in this comparison has been modified so as to use the same second order derivative formula as Stergioulas \& Friedman, to smooth out the oscillations in the metric potential $\alpha$.

\subsubsection{The BGSM code}

Bonazzola et al. (1993) have developed a new formulation based on the $3+1$ formalism which has been used in hydrodynamics in general relativity. Their choice of slicing and gauge in the $3+1$ formalism results in the same form of the metric usually chosen for stationary problems, i.e. (2). Consequently the Einstein equations are reduced to the same differential equations of elliptic type as used by other schemes.

The main part of the BGSM formulation is similar to that of the KEH formulation, except for the metric coefficient $\zeta=\alpha+\nu$ for which a second-order (elliptic) equation is used instead of a first-order equation in $\mathrm{KEH}$. The Einstein equations are reorganized so as to "pick" out the Laplacian operators in two and three dimensional flat spaces and regard all other remaining terms as "source terms" in the Poisson-like equations in two and three dimensional flat spaces. Concerning the matter, essentially the same equation as (8) is used for the hydrostationary equation.

The characteristic features of the BGSM code can be found in the numerical solving method, i.e. the pseudospectral method (Gottlieb \& Orszag 1977; Bonazzola et al. 1996, 1997, 1998b). In the spectral method all functions are expanded in terms of certain base functions and algebraic equations for coefficients which appear in the expansion are solved. Therefore there are two distinct procedures in this method: one is obtaining coefficients from the functions and the other is constructing functions by using the coefficients. Since these two steps need to be, in general, performed many times, it is highly desirable to use a fast algorithm. In the spectral method of the BGSM code, Bonazzola et al. (1993) have adopted trigonometric functions for the angle variable and the Chebyshev polynomials for the radial variable. Consequently for the angle part of any function, the fast fourier transform (FFT) can be employed. Concerning the radial variable, a new variable which is related to the radial variable by a simple equation is introduced so that the Chebyshev polynomials are expressed by the trigonometric functions. After this transformation, one can use the FFT also for the radial variable.

The BGSM code can handle the region extended to infinity as is done by the $\mathrm{KEH}(\mathrm{SF})$ code. This can be performed by introducing a new radial variable $u$ as follows: $u \equiv \frac{R_{0}}{r}, \quad$ for outside of the matter

where $R_{0}$ is the maximum value of the stellar radius. The boundary conditions at infinity, are easily applied at $u=0$.

It may be fair to note that in the Newtonian rotating star problems a similar expansion was used by Ostriker \& Mark (1968) in the SCF method, although Ostriker and Mark used the integral form of the Newtonian potential instead of solving the Poisson equation directly and they did not use the FFT.

\subsection{Relations among the three different codes}

Here we summarize similarities and difference between the three codes:

A) Common features through all three codes:

1. Quasi-isotropic coordinates are used. It implies that the metric components are essentially the same, although background views deriving the metric are not the same.

2. Integral form of the hydrostationary equation for the matter is used.

3. Poisson-like operators are "picked" out from the Einstein equations and the other terms are treated as "source terms".

B) The $\mathrm{KEH}(\mathrm{OR})$ code differs from the other two codes in that the boundary conditions are not applied at infinity, but approximate boundary conditions are applied at a finite distance from the star.

C) A difference between the BGSM code and the other codes is the use of a second-order (elliptic)equation for $\zeta$ in BGSM versus a first-order equation for $\alpha=\zeta-\nu$ in $\mathrm{KEH}$.

We reorganized our codes so as to make the differences as small as possible. The codes agree exactly on:

1. the physical model parameters by which we can specify a model uniquely,

2. the values of physical constants, and

3. the equation of state of matter. 
Since the three codes use different grids and/or numerical methods for solving the field equations, there will always be a residual difference in the results, even after this reorganization. This residual difference is what we want to determine.

\subsection{Starting point of computations}

\subsubsection{Constants}

Values used in this paper for the velocity of light $c$, the gravitational constant $G$, the mass of the sun $M_{\odot}$ and the baryon mass $m_{\mathrm{B}}$ are as follows:

$$
\begin{aligned}
c & =2.997910^{10} \mathrm{~cm} \mathrm{~s}^{-1}, \\
G & =6.673210^{-8} \mathrm{~g}^{-1} \mathrm{~cm}^{3} \mathrm{~s}^{-2} \\
M_{\odot} & =1.98710^{33} \mathrm{~g}, \\
m_{\mathrm{B}} & =1.6610^{-24} \mathrm{~g} .
\end{aligned}
$$

Note that some of the above constants differ slightly from the ones used in the papers where the three codes were first presented.

\subsubsection{Interpolation of tabulated equation-of-state data}

For realistic equations of state, the energy density, pressure and other thermodynamical quantities are given in tables. Intermediate values need to be obtained by a method of interpolation. We will use two different interpolation schemes, the four-point Lagrange interpolation (hereafter LI) and the cubic Hermite interpolation (HI) (Swesty 1996):

A) Lagrange interpolation.

Let us assume that there is a table which relates the variable $x$ to the variable $y$ at $n$ points, i.e. a set of values $\left(x_{i}, y_{i}\right)$ for $i=1, \ldots, n$ are tabulated. For the LI scheme the interpolated formula $y_{\mathrm{LI}}$ can be expressed as

$y_{\mathrm{LI}}(x) \equiv \sum_{i=1}^{n} y_{i} \frac{p_{i}(x)}{\left.p^{\prime}(x)\right|_{x=x_{i}}}$,

where

$$
\begin{aligned}
p(x) & \equiv\left(x-x_{1}\right)\left(x-x_{2}\right) \ldots\left(x-x_{n}\right), \\
p_{i}(x) & \equiv \frac{p(x)}{\left(x-x_{i}\right)} .
\end{aligned}
$$

The prime denotes the differentiation with respect to the argument. This scheme does not guarantee the values of derivatives at the points in the table. In this paper we use the four point Lagrange interpolation, i.e. $n=4$.

B) Hermite interpolation.

In the Hermite interpolation, the interpolated formula $y_{\mathrm{HI}}$ for $x_{i} \leq x \leq x_{i+1}$ is expressed as

$$
\begin{aligned}
y_{\mathrm{HI}}(x) \equiv & y_{i} h_{0}(w)+y_{i+1} h_{0}(1-w) \\
& +\left(\frac{\mathrm{d} y}{\mathrm{~d} x}\right)_{i}\left(x_{i+1}-x_{i}\right) h_{1}(w) \\
& -\left(\frac{\mathrm{d} y}{\mathrm{~d} x}\right)_{i+1}\left(x_{i+1}-x_{i}\right) h_{1}(1-w),
\end{aligned}
$$

where

$w \equiv \frac{x-x_{i}}{x_{i+1}-x_{i}}$.

Here $h_{0}$ and $h_{1}$ are the cubic Hermite functions defined by

$h_{0}(w) \equiv 2 w^{3}-3 w^{2}+1$,

$h_{1}(w) \equiv w^{3}-2 w^{2}+w$.

It is noted that these cubic Hermite functions are unique polynomials of degree three satisfying the following relations:

$$
\begin{aligned}
& h_{0}(0)=1, \\
& h_{0}(1)=h_{0}^{\prime}(0)=h_{0}^{\prime}(1)=0, \\
& h_{1}^{\prime}(0)=1, \\
& h_{1}(0)=h_{1}(1)=h_{1}^{\prime}(1)=0 .
\end{aligned}
$$

Contrary to the LI, in the Hermite interpolation the values as well as their first derivatives at mesh points are exactly reproduced by the interpolation formula. The main advantage of the Hermite interpolation is that it preserves the thermodynamical consistency (Swesty 1996).

\section{Equations of state}

\subsection{Relativistic polytropes}

We use the following relation as a polytropic equation of state (Tooper 1965):

$$
\begin{aligned}
& \varepsilon=K \frac{\rho^{\gamma}}{\gamma-1}+\rho c^{2} \\
& p=K \rho^{\gamma} \\
& \gamma=1+\frac{1}{N}
\end{aligned}
$$

where $K$ and $N$ are the polytropic constant and polytropic index, respectively, while $\rho$ is the rest mass density.

It should be noted that this equation of state includes the limiting case of $\varepsilon=\rho c^{2}=$ constant, when $\gamma=\infty$ $(N=0)$. The constant density models are also called homogeneous models. For polytropes of index $N<1.0$, the density does not go to zero smoothly at the surface and the first derivatives of the density across the surface are discontinuous. This kind of discontinuity may become the cause of unfavorable behavior of solutions, unless it is treated carefully. For constant density models, the situation is even worse, since the density itself is discontinuous across the surface.

Although polytropic EOSs are not as realistic as tabulated EOSs (but one can reproduce neutron star bulk properties with an $N \simeq 1.0$ polytrope), they are helpful to check numerical codes. Since the hydrostationary equation can be analytically integrated and no additional numerical errors arise in solving it. 


\subsection{Short description of realistic equations of state}

As discussed in the introduction, the main uncertainties about neutron star properties are related to the unknown interactions of the neutron star matter at high density regions. In the last decades, many equations of state have been proposed by considering different kinds of interactions into account. A large collection of representative equations of state were compiled by Arnett \& Bowers (1977), who constructed nonrotating neutron star models and obtained physical quantities for slowly rotating neutron stars. We will choose three equations of state of Arnett \& Bowers' compilation, i.e. equations C, $\mathrm{G}$ and $\mathrm{L}$ according to their notation. Equations $\mathrm{C}, \mathrm{G}$ and L are those derived by Bethe \& Johnson (1974), Canuto \& Chitre (1974) and Pandharipande \& Smith (1975) (see also Pandharipande et al. 1976), respectively. Those equations of state were also used by Friedman et al. (1986) for constructing rapidly rotating relativistic neutron stars models.

In addition to these equations of state we also employ the WFF3 ( $\mathrm{UV}_{14}+\mathrm{TNI}$ ) equation of state by Wiringa et al. (1988), the FPS equation of state by Lorenz et al. (1993), and the equation of state which represents a causal limit (CLES).

Some characteristic features of each equation of state can be summarized as follows.

Bethe - Johnson $I(C)$ : EOS C is of intermediate stiffness. The maximum gravitational mass of a spherical neutron star for this EOS is $1.85 M_{\odot}$. The density range is from $1.7110^{14} \mathrm{~g} \mathrm{~cm}^{-3}$ to $3.2310^{15} \mathrm{~g} \mathrm{~cm}^{-3}$. Hyperons as well as nucleons are taken into account. The interaction is assumed non-relativistic and represented by the modified Reid soft core potential with non-integer parameters. To include the many-body theory, the constrained variational principle is employed. This equation of state is joined to the composite $\operatorname{BBP}\left(\varepsilon / c^{2}>4.310^{11} \mathrm{~g} \mathrm{~cm}^{-3}\right)-\operatorname{BPS}$ $\left(10^{4} \mathrm{~g} \mathrm{~cm}^{-3}<\varepsilon / c^{2}<4.310^{11} \mathrm{~g} \mathrm{~cm}^{-3}\right)-\operatorname{FMT}\left(\varepsilon / c^{2}<\right.$ $\left.10^{4} \mathrm{~g} \mathrm{~cm}^{-3}\right)$. Here BBP, BPS and FMT denote equations of state by Baym et al. (1971a), Baym et al. (1971b) and Feynman et al. (1949), respectively.

Canuto - Chitre $(G)$ : EOS G an extremely soft equation of state. The maximum gravitational mass of a spherical neutron star for this equation of state is $1.36 M_{\odot}$, so this EOS is not acceptable as a realistic candidate for the true EOS of neutron star matter. It is used in this comparison, because it is close to the softest possible realistic EOS consistent with observational constraints. The density range is from $2.3710^{15} \mathrm{~g} \mathrm{~cm}^{-3}$ to 7.23 $10^{15} \mathrm{~g} \mathrm{~cm}^{-3}$. Crystallization of neutrons is included. The interaction is non-relativistic and represented by the modified Reid soft core potential. This equation of state is joined to the composite $\mathrm{PC}\left(710^{14} \mathrm{~g} \mathrm{~cm}^{-3}<\varepsilon / c^{2}<2.4\right.$ $\left.10^{15} \mathrm{~g} \mathrm{~cm}^{-3}\right)-\operatorname{BBP}\left(4.310^{11} \mathrm{~g} \mathrm{~cm}^{-3}<\varepsilon / c^{2}<7\right.$ $\left.10^{14} \mathrm{~g} \mathrm{~cm}^{-3}\right)-\operatorname{BPS}\left(10^{4} \mathrm{~g} \mathrm{~cm}^{-3}<\varepsilon / c^{2}<4.3\right.$ $\left.10^{11} \mathrm{~g} \mathrm{~cm}^{-3}\right)-\operatorname{FMT}\left(\varepsilon / c^{2}<10^{4} \mathrm{~g} \mathrm{~cm}^{-3}\right)$. Here PC denotes the equation of state by Pandharipande (1971).

Pandharipande - Smith (L): EOS L is an extremely stiff EOS. The maximum gravitational mass of a spherical neutron star for this equation of state is $2.70 M_{\odot}$. The density range is larger than $4.38610^{11} \mathrm{~g} \mathrm{~cm}^{-3}$. Compositions consist of neutrons. The interaction is assumed non-relativistic and is represented by the nuclear attraction due to scalar particle exchange. This equation of state is joined to the BPS $\left(10^{4} \mathrm{~g} \mathrm{~cm}^{-3}<\varepsilon / c^{2}<4.4\right.$ $\left.10^{11} \mathrm{~g} \mathrm{~cm}^{-3}\right)-\operatorname{FMT}\left(\varepsilon / c^{2}<10^{4} \mathrm{~g} \mathrm{~cm}^{-3}\right)$.

Wiringa - Fiks - Farbrocini (WFF3): EOS WFF3 (Wiringa et al. 1988) is of intermediate stiffness. At present, the WFF3 equation of state is regarded as one of the best candidates for the high density region. This EOS is an improved version of the equation of state by Friedman \& Pandharipande (1981). The nucleon-nucleon interaction described by a two-body Urbana $\mathrm{UV}_{14}$ potential and the phenomenological three-nucleon TNI interaction are taken into account. Compositions are considered to be neutrons. The maximum gravitational mass of a spherical neutron star for this equation of state is $1.84 M_{\odot}$. Although the usual WFF3 EOS is joined to EOS NV (Negele \& Vautherin 1973), we will also use a different version, in which it is joined to the more modern FPS EOS (Lorenz et al. 1993).

Lorenz - Ravenhall - Pethick (FPS): This EOS is also a modern version of the equation of state by Friedman \& Pandharipande (1981). The nucleon-nucleon interaction described by a two-body Urbana $\mathrm{UV}_{14}$ potential and the phenomenological three-nucleon TNI interaction are taken into account. In the FPS equation of state the Skyrme model is used, where the effective interaction has the spatial character of a two-body delta function plus derivatives. The FPS equation of state can be considered to be an improved version of the BBP equation of state in the region of the lower density.

Causal limit equation of state (CLES): As an extreme case, we consider an equation of state which consists of a causal limit $\operatorname{EOS}\left(\varepsilon=p+\right.$ constant) for $\varepsilon / c^{2}>1.66$ $10^{14} \mathrm{~g} \mathrm{~cm}^{-3}$ and the FPS EOS below that density. The causal limit EOS has the property that, in the interior of the star, the phase velocity of sound is equal to the velocity of light in vacuo, i.e. $v_{\mathrm{s}}=\sqrt{\mathrm{d} p / \mathrm{d} \varepsilon}=c$. 
Table 1. Polytropic models

\begin{tabular}{|c|c|c|c|c|c|c|c|c|c|}
\hline Model & N05sn & N05rn & N05sr & N05mr & N05rr & N075sn & N075rn & N075sr & N075mr \\
\hline$N$ & 0.5 & 0.5 & 0.5 & 0.5 & 0.5 & 0.75 & 0.75 & 0.75 & 0.75 \\
\hline $\bar{\varepsilon}_{\mathrm{c}}$ & $1.00 \mathrm{e}-03$ & $1.00 \mathrm{e}-03$ & $1.20 \mathrm{e}+00$ & $1.04 \mathrm{e}+00$ & $2.00 \mathrm{e}+00$ & $3.00 \mathrm{e}-05$ & $3.00 \mathrm{e}-05$ & $8.00 \mathrm{e}-01$ & $6.50 \mathrm{e}-01$ \\
\hline$r_{\mathrm{p}} / r_{\mathrm{e}}$ & $9.80 \mathrm{e}-01$ & $5.99 \mathrm{e}-01$ & $9.85 \mathrm{e}-01$ & $5.50 \mathrm{e}-01$ & $7.57 \mathrm{e}-01$ & $9.80 \mathrm{e}-01$ & $6.82 \mathrm{e}-01$ & $9.79 \mathrm{e}-01$ & $5.66 \mathrm{e}-01$ \\
\hline $\bar{\Omega}$ & $6.91 \mathrm{e}-03$ & $2.85 \mathrm{e}-02$ & $2.14 \mathrm{e}-01$ & $9.78 \mathrm{e}-01$ & $1.00 \mathrm{e}+00$ & $1.10 \mathrm{e}-03$ & $4.11 \mathrm{e}-03$ & $1.77 \mathrm{e}-01$ & $6.30 \mathrm{e}-01$ \\
\hline & $6.92 \mathrm{e}-03$ & & $2.19 \mathrm{e}-01$ & $9.80 \mathrm{e}-01$ & & & & $1.79 \mathrm{e}-01$ & $6.31 \mathrm{e}-01$ \\
\hline $\bar{M}$ & $6.30 \mathrm{e}-08$ & $9.43 \mathrm{e}-08$ & $1.26 \mathrm{e}-01$ & $1.53 \mathrm{e}-01$ & $1.35 \mathrm{e}-01$ & $3.73 \mathrm{e}-07$ & $4.84 \mathrm{e}-07$ & $1.40 \mathrm{e}-01$ & $1.64 \mathrm{e}-01$ \\
\hline & & & $1.27 \mathrm{e}-01$ & $1.54 \mathrm{e}-01$ & $1.36 \mathrm{e}-01$ & & & $1.41 \mathrm{e}-01$ & $1.65 \mathrm{e}-01$ \\
\hline $\bar{M}_{0}$ & $6.30 \mathrm{e}-08$ & $9.43 \mathrm{e}-08$ & $1.52 \mathrm{e}-01$ & $1.83 \mathrm{e}-01$ & $1.59 \mathrm{e}-01$ & $3.73 \mathrm{e}-07$ & $4.84 \mathrm{e}-07$ & $1.60 \mathrm{e}-01$ & $1.87 \mathrm{e}-01$ \\
\hline $\bar{R}_{\text {circ }}$ & $3.04 \mathrm{e}-02$ & $4.20 \mathrm{e}-02$ & $\begin{array}{l}1.53 \mathrm{e}-01 \\
4.03 \mathrm{e}-01\end{array}$ & $5.35 \mathrm{e}-01$ & $\begin{array}{l}1.00 \mathrm{e}-01 \\
4.03 \mathrm{e}-01\end{array}$ & $1.95 \mathrm{e}-01$ & $2.47 \mathrm{e}-01$ & $5.33 \mathrm{e}-01$ & $\begin{array}{l}7.37 \mathrm{e}-01 \\
7.38 \mathrm{e}-01\end{array}$ \\
\hline $\bar{J}$ & $\begin{array}{l}1.31 \mathrm{e}-13 \\
1.32 \mathrm{e}-13\end{array}$ & $1.47 \mathrm{e}-12$ & $\begin{array}{l}2.16 \mathrm{e}-03 \\
2.21 \mathrm{e}-03\end{array}$ & $1.72 \mathrm{e}-02$ & $1.07 \mathrm{e}-02$ & $\begin{array}{l}4.55 \mathrm{e}-12 \\
4.57 \mathrm{e}-12\end{array}$ & $3.35 \mathrm{e}-11$ & $\begin{array}{l}2.87 \mathrm{e}-03 \\
2.89 \mathrm{e}-03\end{array}$ & $1.75 \mathrm{e}-02$ \\
\hline $\bar{I}$ & $1.90 \mathrm{e}-11$ & $5.17 \mathrm{e}-11$ & $1.01 \mathrm{e}-02$ & $1.76 \mathrm{e}-02$ & $1.07 \mathrm{e}-02$ & $4.16 \mathrm{e}-09$ & $8.15 \mathrm{e}-09$ & $1.62 \mathrm{e}-02$ & $2.77 \mathrm{e}-02$ \\
\hline$T / W$ & $\begin{array}{l}5.18 \mathrm{e}-03 \\
5.20 \mathrm{e}-03\end{array}$ & $1.26 \mathrm{e}-01$ & $\begin{array}{l}5.01 \mathrm{e}-03 \\
5.15 \mathrm{e}-03\end{array}$ & $\begin{array}{l}1.47 \mathrm{e}-01 \\
1.49 \mathrm{e}-01\end{array}$ & $\begin{array}{l}8.76 \mathrm{e}-02 \\
896 \mathrm{e}-02\end{array}$ & $\begin{array}{l}4.93 \mathrm{e}-03 \\
4.96 \mathrm{e}-03\end{array}$ & $8.96 \mathrm{e}-02$ & $5.94 \mathrm{e}-03$ & $1.11 \mathrm{e}-01$ \\
\hline$Z_{\mathrm{p}}$ & $2.10 \mathrm{e}-06$ & $3.10 \mathrm{e}-06$ & $\begin{array}{l}6.32 \mathrm{e}-01 \\
6.42 \mathrm{e}-01\end{array}$ & $\begin{array}{l}9.64 \mathrm{e}-01 \\
9.75 \mathrm{e}-01\end{array}$ & $\begin{array}{l}9.97 \mathrm{e}-01 \\
10.0 \mathrm{e}-01\end{array}$ & $1.94 \mathrm{e}-06$ & $\begin{array}{l}2.55 \mathrm{e}-06 \\
2.56 \mathrm{e}-06\end{array}$ & $\begin{array}{l}4.59 \mathrm{e}-01 \\
4.61 \mathrm{e}-01\end{array}$ & $\begin{array}{l}6.10 \mathrm{e}-01 \\
6.13 \mathrm{e}-01\end{array}$ \\
\hline$Z_{\text {eq }}^{\mathrm{f}}$ & $\begin{array}{l}-2.08 \mathrm{e}-04 \\
-2.09 \mathrm{e}-04\end{array}$ & $-1.19 \mathrm{e}-03$ & $\begin{array}{l}4.10 \mathrm{e}-01 \\
4.15 \mathrm{e}-01\end{array}$ & $\begin{array}{l}-3.62 \mathrm{e}-01 \\
-3.64 \mathrm{e}-01\end{array}$ & $\begin{array}{l}-1.42 \mathrm{e}-01 \\
-1.47 \mathrm{e}-01\end{array}$ & $\begin{array}{l}-2.12 \mathrm{e}-04 \\
-2.13 \mathrm{e}-04\end{array}$ & $-1.01 \mathrm{e}-03$ & $\begin{array}{l}2.61 \mathrm{e}-01 \\
2.65 \mathrm{e}-01\end{array}$ & $\begin{array}{l}-3.19 \mathrm{e}-01 \\
-3.20 \mathrm{e}-01\end{array}$ \\
\hline$Z_{\mathrm{eq}}^{\mathrm{b}}$ & $\begin{array}{l}2.12 \mathrm{e}-04 \\
2.13 \mathrm{e}-04\end{array}$ & $1.20 \mathrm{e}-03$ & $\begin{array}{l}8.66 \mathrm{e}-01 \\
8.90 \mathrm{e}-01\end{array}$ & $\begin{array}{l}2.99 \mathrm{e}+00 \\
3.02 \mathrm{e}+00\end{array}$ & $\begin{array}{l}2.86 \mathrm{e}+00 \\
2.92 \mathrm{e}+00\end{array}$ & $\begin{array}{l}2.16 \mathrm{e}-04 \\
2.17 \mathrm{e}-04\end{array}$ & $1.02 \mathrm{e}-03$ & $\begin{array}{l}6.59 \mathrm{e}-01 \\
6.70 \mathrm{e}-01\end{array}$ & $\begin{array}{l}1.72 \mathrm{e}+00 \\
1.73 \mathrm{e}+00\end{array}$ \\
\hline $\mathrm{e}$ & $\begin{array}{l}1.99 \mathrm{e}-01 \\
2.05 \mathrm{e}-01\end{array}$ & $8.01 \mathrm{e}-01$ & $\begin{array}{l}1.31 \mathrm{e}-01 \\
1.72 \mathrm{e}-01\end{array}$ & $\begin{array}{l}6.95 \mathrm{e}-01 \\
7.02 \mathrm{e}-01\end{array}$ & $\begin{array}{l}5.64 \mathrm{e}-01 \\
5.74 \mathrm{e}-01\end{array}$ & $\begin{array}{l}1.96 \mathrm{e}-01 \\
2.09 \mathrm{e}-01\end{array}$ & $7.32 \mathrm{e}-01$ & $\begin{array}{l}1.66 \mathrm{e}-01 \\
1.97 \mathrm{e}-01\end{array}$ & $\begin{array}{l}7.02 \mathrm{e}-01 \\
7.06 \mathrm{e}-01\end{array}$ \\
\hline
\end{tabular}

\section{Results}

\subsection{Models selected for polytropes}

We have started our comparison project by selecting several representative polytropic models, the parameters of which are shown in Tables 1-2. We have chosen

1. models of very low central density (nearly Newtonian) with slow and rapid rotation,

2. models of high central density (relativistic) with slow and rapid rotation, and

3. models at the maximum mass for each EOS.

Note that, the maximum mass model almost coincides with the maximum angular velocity model, unless there is a large phase transition at densities close to the central density of the maximum mass star (cf. Cook et al. 1994b and Stergioulas \& Friedman 1995). For all EOSs in this comparison the two models almost coincide.

In order to evaluate the performance of our numerical codes for models with discontinuous density distribution, we also compare a number of homogeneous models which cover both highly relativistic and Newtonian, rapidly rotating and nonrotating cases, as shown in Tables 3 and 4 (the contents of these tables will be described in Sect. 5.4).

\subsection{Models selected for realistic equations of state}

As discussed in the previous section, we use six representative realistic EOSs: C, G, L, WFF3+FPS, WFF3+NV and FPS. In addition, we use the causal limit EOS CLES. For each equation of state, we compute several models as shown Tables $5-7$. The models correspond to the maximum mass model, a fast rotating $1.4 M_{\odot}$ model and a nonrotating model for each EOS.

\subsection{Computed quantities}

\subsubsection{Grid and physical quantities}

For this comparison project, $\mathrm{KEH}(\mathrm{OR})$ and $\mathrm{KEH}(\mathrm{SF})$ have used grids with $(71 \times 201)$ and $(261 \times 401)$ (angular $\times$ radial) grid-points. In the equatorial plane, half of the radial grid-points are inside the star. BGSM uses 21 $\times 41$ or $33 \times 65$ grid points (note that the notion of "grid points" is not very significant for a spectral method; the above numbers should better be referred to as the numbers of basis functions employed in the expansions of the physical fields).

Here we summarize the notation of computed physical quantities:

$\varepsilon_{\mathrm{c}} \quad$ Central energy density

$r_{\mathrm{p}} / r_{\mathrm{e}}$ Ratio of polar to equatorial radii

$\Omega \quad$ Angular velocity of the star

$M_{0} \quad$ Baryon mass

$M_{\mathrm{p}} \quad$ Proper mass

$M \quad$ Gravitational mass

$R_{\text {circ }}$ Equatorial circumferential radius 
Table 2. Polytropic models (continued)

\begin{tabular}{|c|c|c|c|c|c|c|c|c|c|c|}
\hline Model & N10sn & N10rn & N10sr & $\mathrm{N} 10 \mathrm{mr}$ & N10rr & N15sn & N15rn & N15sr & $\mathrm{N} 15 \mathrm{mr}$ & N15rr \\
\hline$N$ & 1.0 & 1.0 & 1.0 & 1.0 & 1.0 & 1.5 & 1.5 & 1.5 & 1.5 & 1.5 \\
\hline$\overline{\bar{\varepsilon}_{\mathrm{c}}}$ & $1.00 \mathrm{e}-06$ & $1.00 \mathrm{e}-06$ & $4.00 \mathrm{e}-01$ & $3.40 \mathrm{e}-01$ & $1.00 \mathrm{e}+00$ & $1.00 \mathrm{e}-09$ & $1.00 \mathrm{e}-09$ & $6.50 \mathrm{e}-01$ & $6.10 \mathrm{e}-02$ & $1.50 \mathrm{e}-01$ \\
\hline$r_{\mathrm{p}} / r_{\mathrm{e}}$ & $9.76 \mathrm{e}-01$ & $6.39 \mathrm{e}-01$ & $9.72 \mathrm{e}-01$ & $5.84 \mathrm{e}-01$ & $8.34 \mathrm{e}-01$ & $9.60 \mathrm{e}-01$ & $7.08 \mathrm{e}-01$ & $8.68 \mathrm{e}-01$ & $6.20 \mathrm{e}-01$ & $8.40 \mathrm{e}-01$ \\
\hline $\bar{\Omega}$ & $2.00 \mathrm{e}-04$ & $7.00 \mathrm{e}-04$ & $1.26 \mathrm{e}-01$ & $3.77 \mathrm{e}-01$ & $\begin{array}{l}4.00 \mathrm{e}-01 \\
4.01 \mathrm{e}-01\end{array}$ & $6.53 \mathrm{e}-06$ & $1.58 \mathrm{e}-05$ & $1.61 \mathrm{e}-01$ & $1.11 \mathrm{e}-01$ & $1.20 \mathrm{e}-01$ \\
\hline $\bar{M}$ & $2.54 \mathrm{e}-06$ & $3.29 \mathrm{e}-06$ & $\begin{array}{l}1.65 \mathrm{e}-01 \\
1.66 \mathrm{e}-01\end{array}$ & $\begin{array}{l}1.88 \mathrm{e}-01 \\
1.89 \mathrm{e}-01\end{array}$ & $1.61 \mathrm{e}-01$ & $9.76 \mathrm{e}-05$ & $1.11 \mathrm{e}-04$ & $2.10 \mathrm{e}-01$ & $2.91 \mathrm{e}-01$ & $2.66 \mathrm{e}-01$ \\
\hline $\bar{M}_{0}$ & $2.54 \mathrm{e}-06$ & $3.29 \mathrm{e}-06$ & $1.82 \mathrm{e}-01$ & $2.07 \mathrm{e}-01$ & $1.73 \mathrm{e}-01$ & $9.76 \mathrm{e}-05$ & $1.11 \mathrm{e}-04$ & $2.08 \mathrm{e}-01$ & $3.04 \mathrm{e}-01$ & $2.77 \mathrm{e}-01$ \\
\hline $\bar{R}_{\text {circ }}$ & $1.27 \mathrm{e}+00$ & $1.71 \mathrm{e}+00$ & $7.82 \mathrm{e}-01$ & $1.09 \mathrm{e}+00$ & $6.79 \mathrm{e}-01$ & $5.29 \mathrm{e}+01$ & $6.63 \mathrm{e}+01$ & $1.27 \mathrm{e}+00$ & $2.85 \mathrm{e}+00$ & $1.79 \mathrm{e}+00$ \\
\hline $\bar{J}$ & $2.14 \mathrm{e}-10$ & $1.50 \mathrm{e}-09$ & $\begin{array}{l}4.24 \mathrm{e}-03 \\
4.25 \mathrm{e}-03\end{array}$ & $2.02 \mathrm{e}-02$ & $9.48 \mathrm{e}-03$ & $3.60 \mathrm{e}-07$ & $1.26 \mathrm{e}-06$ & $1.02 \mathrm{e}-02$ & $3.88 \mathrm{e}-02$ & $2.13 \mathrm{e}-02$ \\
\hline $\bar{I}$ & $1.07 \mathrm{e}-06$ & $2.14 \mathrm{e}-06$ & $3.36 \mathrm{e}-02$ & $\begin{array}{l}5.36 \mathrm{e}-02 \\
5.37 \mathrm{e}-02\end{array}$ & $2.37 \mathrm{e}-02$ & $5.51 \mathrm{e}-02$ & $\begin{array}{l}7.99 \mathrm{e}-02 \\
8.00 \mathrm{e}-02\end{array}$ & $\begin{array}{l}6.32 \mathrm{e}-02 \\
6.33 \mathrm{e}-02\end{array}$ & $3.50 \mathrm{e}-01$ & $1.78 \mathrm{e}-01$ \\
\hline$T / W$ & $\begin{array}{l}5.56 \mathrm{e}-03 \\
5.57 \mathrm{e}-03\end{array}$ & $9.10 \mathrm{e}-02$ & $\begin{array}{l}6.61 \mathrm{e}-03 \\
6.63 \mathrm{e}-03\end{array}$ & $\begin{array}{l}8.36 \mathrm{e}-02 \\
8.41 \mathrm{e}-02\end{array}$ & $\begin{array}{l}3.48 \mathrm{e}-02 \\
3.52 \mathrm{e}-02\end{array}$ & $7.48 \mathrm{e}-03$ & $5.24 \mathrm{e}-02$ & $1.32 \mathrm{e}-02$ & $\begin{array}{l}4.75 \mathrm{e}-02 \\
4.76 \mathrm{e}-02\end{array}$ & $\begin{array}{l}2.29 \mathrm{e}-02 \\
2.30 \mathrm{e}-02\end{array}$ \\
\hline$Z_{\mathrm{p}}$ & $\begin{array}{l}2.04 \mathrm{e}-06 \\
2.05 \mathrm{e}-06\end{array}$ & $\begin{array}{l}2.71 \mathrm{e}-06 \\
2.74 \mathrm{e}-06\end{array}$ & $\begin{array}{l}3.23 \mathrm{e}-01 \\
3.26 \mathrm{e}-01\end{array}$ & $\begin{array}{l}4.04 \mathrm{e}-01 \\
4.05 \mathrm{e}-01\end{array}$ & $\begin{array}{l}4.56 \mathrm{e}-01 \\
4.58 \mathrm{e}-01\end{array}$ & $1.91 \mathrm{e}-06$ & $\begin{array}{l}2.25 \mathrm{e}-06 \\
2.27 \mathrm{e}-06\end{array}$ & $\begin{array}{l}2.57 \mathrm{e}-01 \\
2.58 \mathrm{e}-01\end{array}$ & $\begin{array}{l}1.94 \mathrm{e}-01 \\
1.95 \mathrm{e}-01\end{array}$ & $\begin{array}{l}2.29 \mathrm{e}-01 \\
2.30 \mathrm{e}-01\end{array}$ \\
\hline$Z_{\text {eq }}^{\mathrm{f}}$ & $-2.52 \mathrm{e}-04$ & $-1.19 \mathrm{e}-03$ & $\begin{array}{l}1.54 \mathrm{e}-01 \\
1.56 \mathrm{e}-01\end{array}$ & $\begin{array}{l}-2.83 e-01 \\
-2.84 e-01\end{array}$ & $\begin{array}{l}-5.95 \mathrm{e}-02 \\
-6.19 \mathrm{e}-02\end{array}$ & $-3.44 \mathrm{e}-04$ & $-1.05 \mathrm{e}-03$ & $\begin{array}{l}-5.33 \mathrm{e}-02 \\
-5.43 \mathrm{e}-02\end{array}$ & $\begin{array}{l}-2.24 \mathrm{e}-01 \\
-2.25 \mathrm{e}-01\end{array}$ & $\begin{array}{l}-8.10 \mathrm{e}-02 \\
-8.17 \mathrm{e}-02\end{array}$ \\
\hline$Z_{\mathrm{eq}}^{\mathrm{b}}$ & $\begin{array}{l}2.56 \mathrm{e}-04 \\
2.57 \mathrm{e}-04\end{array}$ & $1.20 \mathrm{e}-03$ & $\begin{array}{l}4.97 \mathrm{e}-01 \\
5.02 \mathrm{e}-01\end{array}$ & $1.15 \mathrm{e}+00$ & $\begin{array}{l}1.03 \mathrm{e}+00 \\
1.04 \mathrm{e}+00\end{array}$ & $3.48 \mathrm{e}-04$ & $1.05 \mathrm{e}-03$ & $\begin{array}{l}5.75 \mathrm{e}-01 \\
5.78 \mathrm{e}-01\end{array}$ & $6.23 \mathrm{e}-01$ & $\begin{array}{l}5.49 \mathrm{e}-01 \\
5.51 \mathrm{e}-01\end{array}$ \\
\hline e & $\begin{array}{l}2.16 \mathrm{e}-01 \\
2.27 \mathrm{e}-01 \\
\end{array}$ & $7.70 \mathrm{e}-01$ & $\begin{array}{l}2.03 \mathrm{e}-01 \\
2.25 \mathrm{e}-01 \\
\end{array}$ & $\begin{array}{l}7.14 \mathrm{e}-01 \\
7.18 \mathrm{e}-01 \\
\end{array}$ & $\begin{array}{l}4.75 \mathrm{e}-01 \\
4.85 \mathrm{e}-01 \\
\end{array}$ & $\begin{array}{l}2.80 \mathrm{e}-01 \\
2.84 \mathrm{e}-01 \\
\end{array}$ & $\begin{array}{l}7.06 \mathrm{e}-01 \\
7.07 \mathrm{e}-01 \\
\end{array}$ & $\begin{array}{l}4.44 \mathrm{e}-01 \\
4.52 \mathrm{e}-01 \\
\end{array}$ & $7.30 \mathrm{e}-01$ & $\begin{array}{l}4.93 \mathrm{e}-01 \\
5.00 \mathrm{e}-01 \\
\end{array}$ \\
\hline
\end{tabular}

$r_{\mathrm{e}} \quad$ Equatorial coordinate radius

$J \quad$ Total angular momentum

I Moment of inertia about the rotation axis

$T \quad$ Rotational energy

$W \quad$ Gravitational energy

$v_{\mathrm{e}} \quad$ Velocity of comoving observer at the equator relative to the locally non-rotating observer

$Z_{\mathrm{p}} \quad$ Polar redshift

$Z_{\mathrm{c}} \quad$ Central redshift

$Z_{\mathrm{eq}}^{\mathrm{b}} \quad$ Equatorial redshift in the backward direction

$Z_{\text {eq }}^{\mathrm{f}} \quad$ Equatorial redshift in the forward direction

e Intrinsic eccentricity of the star's surface

$G R V 2$ Two dimensional virial identity

$G R V 3$ Three dimensional virial identity.

Some of the quantities in the above list can be expressed as follows:

$Z_{\mathrm{p}}=\mathrm{e}^{-2 \nu_{\mathrm{p}}}-1$,

$Z_{\mathrm{eq}}^{\mathrm{f}}=\left(\frac{1-v_{\mathrm{e}}}{1+v_{\mathrm{e}}}\right)^{1 / 2} \frac{\mathrm{e}^{-\nu_{\mathrm{e}}}}{1+r_{\mathrm{e}} \mathrm{e}^{\left(\nu_{\mathrm{e}}-\beta_{\mathrm{e}}\right) / 2} \omega_{\mathrm{e}}}-1$,

$Z_{\mathrm{eq}}^{\mathrm{b}}=\left(\frac{1+v_{\mathrm{e}}}{1-v_{\mathrm{e}}}\right)^{1 / 2} \frac{\mathrm{e}^{-\nu_{\mathrm{e}}}}{1-r_{\mathrm{e}} \mathrm{e}^{\left(\nu_{\mathrm{e}}-\beta_{\mathrm{e}}\right) / 2} \omega_{\mathrm{e}}}-1$,

where subscripts $\mathrm{p}$ and $\mathrm{e}$ denote values at the pole and the equatorial surface, respectively.

$M_{0}=2 \pi \int \rho \frac{\mathrm{e}^{2 \alpha+\beta}}{\sqrt{1-v^{2}}} r^{2} \sin \theta \mathrm{d} r \mathrm{~d} \theta$

$M_{\mathrm{p}}=2 \pi \int \varepsilon \frac{\mathrm{e}^{2 \alpha+\beta}}{\sqrt{1-v^{2}}} r^{2} \sin \theta \mathrm{d} r \mathrm{~d} \theta$

$$
\begin{aligned}
& M=2 \pi \int\left[\mathrm{e}^{2 \alpha+\beta}\left\{\frac{(\varepsilon+p)\left(1+v^{2}\right)}{1-v^{2}}+2 p\right\}\right. \\
& \left.+2 r \sin \theta \omega \mathrm{e}^{\beta} \frac{(\varepsilon+p) v}{1-v^{2}}\right] r^{2} \sin \theta \mathrm{d} r \mathrm{~d} \theta, \\
& J=2 \pi \int \mathrm{e}^{2 \alpha+2 \beta} \frac{(\varepsilon+p) v}{1-v^{2}} r^{3} \sin ^{2} \theta \mathrm{d} r \mathrm{~d} \theta, \\
& T=\frac{1}{2} \int \Omega \mathrm{d} J=2 \pi \int \mathrm{e}^{2 \alpha+2 \beta} \frac{(\varepsilon+p) v}{1-v^{2}} \Omega r^{3} \sin ^{2} \theta \mathrm{d} r \mathrm{~d} \theta, \\
& W=M_{\mathrm{p}} c^{2}+T-M c^{2}, \\
& I=\frac{J}{\Omega} \text {. }
\end{aligned}
$$

The eccentricity of the meridional cross section is defined by the following procedure (Friedman et al. 1986). If the surface of the star is defined by

$r=r_{\mathrm{s}}(\theta)$,

the metric of the stellar surface can be expressed as

$\mathrm{d} \sigma^{2}=\mathrm{e}^{2 \beta} r^{2} \sin ^{2} \theta \mathrm{d} \varphi^{2}+\mathrm{e}^{2 \alpha}\left[\left(\frac{\mathrm{d} r_{\mathrm{s}}}{\mathrm{d} \theta}\right)^{2}+r_{\mathrm{s}}(\theta)^{2}\right] \mathrm{d} \theta^{2}$

If we embed this surface in the flat three dimensional space, it is expressed as

$R=R_{\mathrm{s}}(z)$,

in cylindrical coordinates $(R, \varphi, z)$. The 2-metric of this surface is

$\mathrm{d} \sigma^{2}=\left[\left(\frac{\mathrm{d} R_{\mathrm{s}}}{\mathrm{d} z}\right)^{2}+1\right] \mathrm{d} z^{2}+R_{\mathrm{s}}^{2} \mathrm{~d} \varphi^{2}$ 
Table 3. Spherical constant density models

\begin{tabular}{ccc}
\hline Model & N00sn & N00sr \\
\hline $\bar{\varepsilon}_{\mathrm{c}}$ & $1.00 \mathrm{e}+00$ & $1.00 \mathrm{e}+00$ \\
$\bar{p}_{\mathrm{c}}$ & $1.00 \mathrm{e}-04$ & $1.00 \mathrm{e}+00$ \\
$r_{\mathrm{p}} / r_{\mathrm{e}}$ & $1.00 \mathrm{e}+00$ & $1.00 \mathrm{e}+00$ \\
$\bar{M}$ & $1.38 \mathrm{e}-06$ & $1.12 \mathrm{e}-01$ \\
& $1.41 \mathrm{e}-06$ & $1.13 \mathrm{e}-01$ \\
$\bar{M}_{0}$ & $1.38 \mathrm{e}-06$ & $1.59 \mathrm{e}-01$ \\
& $1.41 \mathrm{e}-06$ & $1.61 \mathrm{e}-01$ \\
$\bar{R}_{\text {circ }}$ & $6.91 \mathrm{e}-03$ & $2.99 \mathrm{e}-01$ \\
& $6.93 \mathrm{e}-03$ & $3.00 \mathrm{e}-01$ \\
$Z_{\mathrm{p}}$ & $2.00 \mathrm{e}-04$ & $9.71 \mathrm{e}-01$ \\
& $2.04 \mathrm{e}-04$ & $10.1 \mathrm{e}-01$ \\
$Z_{\text {eq }}^{\mathrm{f}}$ & $2.00 \mathrm{e}-04$ & $9.71 \mathrm{e}-01$ \\
& $2.04 \mathrm{e}-04$ & $10.1 \mathrm{e}-01$ \\
$Z_{\text {eq }}^{\mathrm{b}}$ & $2.00 \mathrm{e}-04$ & $9.71 \mathrm{e}-01$ \\
& $2.04 \mathrm{e}-04$ & $10.1 \mathrm{e}-01$ \\
\hline
\end{tabular}

Comparing these two equations, we have the following relations, if they express the same surface geometry:

$R_{\mathrm{s}}(\theta)=\mathrm{e}^{\beta} r \sin \theta$

and

$$
\begin{gathered}
z_{\mathrm{s}}(\theta)=\int_{\theta}^{\pi / 2} \mathrm{~d} \theta\left\{\mathrm{e}^{2 \alpha}\left[\left(\frac{\mathrm{d} r_{\mathrm{s}}}{\mathrm{d} \theta}\right)^{2}+r_{\mathrm{s}}(\theta)^{2}\right]\right. \\
\left.-\left(\frac{\mathrm{d} R_{\mathrm{s}}}{\mathrm{d} \theta}\right)^{2}\right\}^{1 / 2} .
\end{gathered}
$$

Using these quantities the eccentricity is defined as

$\mathrm{e} \equiv \sqrt{1-\left(\frac{z_{\mathrm{s}}(\theta=0)}{R_{\mathrm{s}}(\theta=\pi / 2)}\right)^{2}}$.

For polytropes, it is convenient to express quantities in dimensionless form, by using $K^{N / 2}$ as a fundamental length scale, as was done in Cook et al. (1994a). In geometrized units $(c=G=1)$, dimensionless quantities are define as follows:

$$
\begin{aligned}
\bar{r} & \equiv K^{-N / 2} r, \\
\bar{R}_{\text {circ }} & \equiv K^{-N / 2} R_{\text {circ }}, \\
\bar{\Omega} & \equiv K^{N / 2} \Omega, \\
\bar{\varepsilon} & \equiv K^{N} \varepsilon, \\
\bar{p} & \equiv K^{N} p, \\
\bar{\rho} & \equiv K^{N} \rho, \\
\bar{J} & \equiv K^{-N} J \\
\bar{I} & \equiv K^{-3 N / 2} J, \\
\bar{M} & \equiv K^{-N / 2} M, \\
\bar{M}_{0} & \equiv K^{-N / 2} M_{0} .
\end{aligned}
$$

\subsubsection{Virial theorem}

Equilibrium configurations in Newtonian gravity satisfy the following relation:

$2 T+3(\gamma-1) U+W=0$,
Table 4. Rotating constant density models

\begin{tabular}{ccc}
\hline Model & N00rn & N00rr \\
\hline $\bar{\varepsilon}_{\mathrm{c}}$ & $1.00 \mathrm{e}+00$ & $1.00 \mathrm{e}+00$ \\
$\bar{p}_{\mathrm{c}}$ & $1.00 \mathrm{e}-04$ & $1.00 \mathrm{e}+00$ \\
$r_{\mathrm{p}} / r_{\mathrm{e}}$ & $6.50 \mathrm{e}-01$ & $7.00 \mathrm{e}-01$ \\
$\bar{\Omega}$ & $1.00 \mathrm{e}+00$ & $1.40 \mathrm{e}+00$ \\
& $1.02 \mathrm{e}+00$ & $1.41 \mathrm{e}+00$ \\
$\bar{M}$ & $2.05 \mathrm{e}-06$ & $1.35 \mathrm{e}-01$ \\
& $2.06 \mathrm{e}-06$ & $1.39 \mathrm{e}-01$ \\
$\bar{M}_{0}$ & $2.05 \mathrm{e}-06$ & $1.84 \mathrm{e}-01$ \\
& $2.06 \mathrm{e}-06$ & $1.87 \mathrm{e}-01$ \\
$\bar{R}_{\text {circ }}$ & $9.06 \mathrm{e}-03$ & $3.45 \mathrm{e}-01$ \\
& $9.11 \mathrm{e}-03$ & $3.46 \mathrm{e}-01$ \\
$\bar{J}$ & $6.76 \mathrm{e}-11$ & $1.37 \mathrm{e}-02$ \\
& $6.97 \mathrm{e}-11$ & $1.41 \mathrm{e}-02$ \\
$\bar{I}$ & $6.74 \mathrm{e}-11$ & $9.82 \mathrm{e}-03$ \\
& $6.87 \mathrm{e}-11$ & $10.0 \mathrm{e}-03$ \\
$T / W$ & $1.08 \mathrm{e}-01$ & $1.63 \mathrm{e}-01$ \\
& $1.14 \mathrm{e}-01$ & $1.68 \mathrm{e}-01$ \\
$Z_{\mathrm{p}}$ & $2.83 \mathrm{e}-04$ & $1.60 \mathrm{e}+00$ \\
& $2.86 \mathrm{e}-04$ & $1.71 \mathrm{e}+00$ \\
$Z_{\mathrm{eq}}^{\mathrm{f}}$ & $-8.81 \mathrm{e}-03$ & $-1.55 \mathrm{e}-01$ \\
& $-8.96 \mathrm{e}-03$ & $-1.60 \mathrm{e}-01$ \\
$Z_{\mathrm{eq}}^{\mathrm{b}}$ & $9.38 \mathrm{e}-03$ & $9.41 \mathrm{e}+00$ \\
& $9.54 \mathrm{e}-03$ & $11.3 \mathrm{e}+00$ \\
$\mathrm{e}$ & $7.60 \mathrm{e}-01$ & $7.07 \mathrm{e}-01$ \\
& $7.61 \mathrm{e}-01$ & $7.11 \mathrm{e}-01$ \\
\hline & &
\end{tabular}

where $U$ is the internal energy. This relation is called the virial relation and has been used to check the accuracy of numerically obtained solutions (see e.g. Ostriker \& Mark 1968; Tassoul 1978).

In general relativity, similar relations were first found by Bonazzola (1973). Recently, two virial identities in general relativity have been discovered by Gourgoulhon \& Bonazzola (1994) and Bonazzola \& Gourgoulhon (1994). Those identities are valid for a general asymptotically flat spacetime. We can use these identities to estimate the numerical error. Let us define two quantities $\lambda_{2}$ and $\lambda_{3}$ as follows:

$\lambda_{2} \equiv \frac{8 \pi \int_{0}^{+\infty} \int_{0}^{\pi}\left[p+(\varepsilon+p) \frac{v^{2}}{1-v^{2}}\right] \mathrm{e}^{2 \mu} r \mathrm{~d} r \mathrm{~d} \theta}{\int_{0}^{+\infty} \int_{0}^{\pi}\left[\partial \nu \partial \nu-\frac{3}{4} \mathrm{e}^{2 \psi-2 \nu} \partial \omega \partial \omega\right] r \mathrm{~d} r \mathrm{~d} \theta}$

and

$$
\begin{aligned}
\lambda_{3} \equiv & 4 \pi \int_{0}^{+\infty} \int_{0}^{\pi}\left[3 p+(\varepsilon+p) \frac{v^{2}}{1-v^{2}}\right] \mathrm{e}^{2 \mu+\psi} r \mathrm{~d} r \mathrm{~d} \theta \\
& \times\left\{\int _ { 0 } ^ { + \infty } \int _ { 0 } ^ { \pi } \left[\partial \nu \partial \nu-\frac{1}{2} \partial \mu \partial \psi\right.\right. \\
& +\frac{\mathrm{e}^{2 \mu-2 \psi}}{2} r \sin ^{2} \theta\left(\frac{\partial \mu}{\partial r}+\frac{1}{r \tan \theta} \frac{\partial \mu}{\partial \theta}\right) \\
& +\frac{1}{4 r}\left(1-\mathrm{e}^{2 \mu-2 \psi} r^{2} \sin ^{2} \theta\right)\left(\frac{\partial \psi}{\partial r}+\frac{1}{r \tan \theta} \frac{\partial \psi}{\partial \theta}\right.
\end{aligned}
$$


Table 5. Spherical models with realistic EOSs

\begin{tabular}{cccccccc}
\hline Model & Csr & Gsr & Lsr & WFF(FPS)sr WFF(NV)sr & FPSsr & CLESsr \\
EOS & C & G & L & WFF3+FPS & WFF3+NV & FPS & CLES \\
\hline$\varepsilon_{\mathrm{c}}\left[\mathrm{g} \mathrm{cm}^{-3}\right]$ & $1.09 \mathrm{e}+15$ & $6.31 \mathrm{e}+15$ & $4.30 \mathrm{e}+14$ & $1.22 \mathrm{e}+15$ & $1.22 \mathrm{e}+15$ & $1.31 \mathrm{e}+15$ & $1.85 \mathrm{e}+14$ \\
$r_{\mathrm{p}} / r_{\mathrm{e}}$ & $1.00 \mathrm{e}+00$ & $1.00 \mathrm{e}+00$ & $1.00 \mathrm{e}+00$ & $1.00 \mathrm{e}+00$ & $1.00 \mathrm{e}+00$ & $1.00 \mathrm{e}+00$ & $1.00 \mathrm{e}+00$ \\
$M\left[M_{\odot}\right]$ & $1.41 \mathrm{E}+00$ & $1.36 \mathrm{E}+00$ & $1.39 \mathrm{e}+00$ & $1.41 \mathrm{e}+00$ & $1.41 \mathrm{e}+00$ & $1.41 \mathrm{e}+00$ & $1.41 \mathrm{e}+00$ \\
& & $1.37 \mathrm{E}+00$ & $1.41 \mathrm{e}+00$ & & & & \\
$M_{0}\left[M_{\odot}\right]$ & $1.55 \mathrm{e}+00$ & $1.57 \mathrm{e}+00$ & $1.49 \mathrm{e}+00$ & $1.57 \mathrm{e}+00$ & $1.57 \mathrm{e}+00$ & $1.57 \mathrm{e}+00$ & $1.51 \mathrm{e}+00$ \\
& & & $1.53 \mathrm{e}+00$ & & & & \\
$R_{\text {circ }}[\mathrm{km}]$ & $1.19 \mathrm{e}+01$ & $6.94 \mathrm{e}+00$ & $1.48 \mathrm{e}+01$ & $1.09 \mathrm{e}+01$ & $1.09 \mathrm{e}+01$ & $1.08 \mathrm{e}+01$ & $1.77 \mathrm{e}+01$ \\
$Z_{\mathrm{p}}$ & $2.39 \mathrm{e}-01$ & $5.28 \mathrm{e}-01$ & $1.76 \mathrm{e}-01$ & $2.69 \mathrm{e}-01$ & $2.68 \mathrm{e}-01$ & $2.72 \mathrm{e}-01$ & $1.43 \mathrm{e}-01$ \\
& $2.41 \mathrm{e}-01$ & $5.38 \mathrm{e}-01$ & $1.80 \mathrm{e}-01$ & $2.71 \mathrm{e}-01$ & $2.71 \mathrm{e}-01$ & $2.74 \mathrm{e}-01$ & $1.44 \mathrm{e}-01$ \\
$Z_{\mathrm{eq}}^{\mathrm{f}}$ & $2.39 \mathrm{e}-01$ & $5.28 \mathrm{e}-01$ & $1.76 \mathrm{e}-01$ & $2.69 \mathrm{e}-01$ & $2.68 \mathrm{e}-01$ & $2.72 \mathrm{e}-01$ & $1.43 \mathrm{e}-01$ \\
& $2.41 \mathrm{e}-01$ & $5.38 \mathrm{e}-01$ & $1.80 \mathrm{e}-01$ & $2.71 \mathrm{e}-01$ & $2.71 \mathrm{e}-01$ & $2.74 \mathrm{e}-01$ & $1.44 \mathrm{e}-01$ \\
$Z_{\mathrm{eq}}^{\mathrm{b}}$ & $2.39 \mathrm{e}-01$ & $5.28 \mathrm{e}-01$ & $1.76 \mathrm{e}-01$ & $2.69 \mathrm{e}-01$ & $2.68 \mathrm{e}-01$ & $2.72 \mathrm{e}-01$ & $1.43 \mathrm{e}-01$ \\
& $2.41 \mathrm{e}-01$ & $5.38 \mathrm{e}-01$ & $1.80 \mathrm{e}-01$ & $2.71 \mathrm{e}-01$ & $2.71 \mathrm{e}-01$ & $2.74 \mathrm{e}-01$ & $1.44 \mathrm{e}-01$ \\
\hline
\end{tabular}

Table 6. Rotating models with realistic EOSs

\begin{tabular}{|c|c|c|c|c|c|c|c|c|}
\hline $\begin{array}{c}\text { Model } \\
\text { EOS }\end{array}$ & $\begin{array}{c}\text { Cbr } \\
\mathrm{C} \\
\end{array}$ & $\begin{array}{c}\mathrm{Cmr} \\
\mathrm{C}\end{array}$ & $\begin{array}{c}\text { Gbr } \\
\text { G }\end{array}$ & $\begin{array}{c}\mathrm{Gmr} \\
\mathrm{G}\end{array}$ & $\begin{array}{c}\text { Lbr } \\
\text { L }\end{array}$ & $\begin{array}{c}\mathrm{L}(\mathrm{L}) \mathrm{mr} \\
\mathrm{L}(\mathrm{LI})\end{array}$ & $\begin{array}{c}\mathrm{L}(\mathrm{H}) \mathrm{mr} \\
\mathrm{L}(\mathrm{HI})\end{array}$ & $\begin{array}{l}\text { WFF(FPS)br } \\
\text { WFF3+FPS }\end{array}$ \\
\hline$\varepsilon_{\mathrm{c}}\left[\mathrm{g} \mathrm{cm}^{-3}\right]$ & $8.70 \mathrm{e}+14$ & $2.64 \mathrm{e}+15$ & $3.10 \mathrm{e}+15$ & $5.58 \mathrm{e}+15$ & $3.90 \mathrm{e}+14$ & $1.20 \mathrm{e}+15$ & $1.20 \mathrm{e}+15$ & $9.70 \mathrm{e}+14$ \\
\hline$r_{\mathrm{p}} / r_{\mathrm{e}}$ & $6.73 \mathrm{e}-01$ & $5.72 \mathrm{e}-01$ & $6.37 \mathrm{e}-01$ & $5.77 \mathrm{e}-01$ & $7.08 \mathrm{e}-01$ & $5.53 \mathrm{e}-01$ & $5.53 e-01$ & $6.30 \mathrm{e}-01$ \\
\hline$\Omega\left[10^{4} \mathrm{~s}^{-1}\right]$ & $5.89 \mathrm{e}-01$ & $1.07 \mathrm{e}+00$ & $1.16 \mathrm{e}+00$ & $1.57 \mathrm{e}+00$ & $4.21 \mathrm{e}-01$ & $8.17 \mathrm{e}-01$ & $8.13 \mathrm{e}-01$ & $7.00 \mathrm{e}-01$ \\
\hline & & & & $1.58 \mathrm{e}+00$ & $4.25 \mathrm{e}-01$ & $8.18 \mathrm{e}-01$ & $8.14 \mathrm{e}-01$ & $7.01 \mathrm{e}-01$ \\
\hline$M\left[M_{\odot}\right]$ & $1.41 \mathrm{e}+00$ & $2.17 \mathrm{e}+00$ & $1.41 \mathrm{e}+00$ & $1.57 \mathrm{e}+00$ & $\begin{array}{l}1.41 \mathrm{e}+00 \\
1.43 \mathrm{e}+00\end{array}$ & $\begin{array}{l}3.31 \mathrm{e}+00 \\
3.33 \mathrm{e}+00\end{array}$ & $\begin{array}{l}3.29 \mathrm{e}+00 \\
3.30 \mathrm{e}+00\end{array}$ & $1.42 \mathrm{e}+00$ \\
\hline$M_{0}\left[M_{\odot}\right]$ & $\begin{array}{l}1.52 \mathrm{e}+00 \\
1.53 \mathrm{e}+00\end{array}$ & $\begin{array}{l}2.48 \mathrm{e}+00 \\
2.49 \mathrm{e}+00\end{array}$ & $\begin{array}{l}1.58 \mathrm{e}+00 \\
1.59 \mathrm{e}+00\end{array}$ & $1.79 \mathrm{e}+00$ & $\begin{array}{l}1.50 \mathrm{e}+00 \\
1.53 \mathrm{e}+00\end{array}$ & $\begin{array}{l}3.90 \mathrm{e}+00 \\
3.92 \mathrm{e}+00\end{array}$ & $\begin{array}{l}3.87 \mathrm{e}+00 \\
3.88 \mathrm{e}+00\end{array}$ & $1.55 \mathrm{e}+00$ \\
\hline$R_{\text {circ }}[\mathrm{km}]$ & $1.52 \mathrm{e}+01$ & $\begin{array}{l}1.32 \mathrm{e}+01 \\
1.34 \mathrm{e}+01\end{array}$ & $1.02 \mathrm{e}+01$ & $\begin{array}{l}9.24 \mathrm{e}+00 \\
9.25 \mathrm{e}+00\end{array}$ & $\begin{array}{l}1.76 \mathrm{e}+01 \\
1.77 \mathrm{e}+01\end{array}$ & $1.85 \mathrm{e}+01$ & $1.85 \mathrm{e}+01$ & $1.43 \mathrm{e}+01$ \\
\hline$c J / G M_{\odot}^{2}$ & $1.20 \mathrm{e}+00$ & $\begin{array}{l}2.99 \mathrm{e}+00 \\
3.00 \mathrm{e}+00\end{array}$ & $1.19 \mathrm{e}+00$ & $1.54 \mathrm{e}+00$ & $\begin{array}{l}1.24 \mathrm{e}+00 \\
1.28 \mathrm{e}+00\end{array}$ & $\begin{array}{l}7.72 \mathrm{e}+00 \\
7.80 \mathrm{e}+00\end{array}$ & $\begin{array}{l}7.62 \mathrm{e}+00 \\
7.64 \mathrm{e}+00\end{array}$ & $1.31 \mathrm{e}+00$ \\
\hline$I\left[10^{45} \mathrm{~g} \mathrm{~cm}^{2}\right]$ & $1.80 \mathrm{e}+00$ & $\begin{array}{l}2.45 \mathrm{e}+00 \\
2.46 \mathrm{e}+00\end{array}$ & $\begin{array}{l}9.01 \mathrm{e}-01 \\
9.03 \mathrm{e}-01\end{array}$ & $\begin{array}{l}8.56 \mathrm{e}-01 \\
8.59 \mathrm{e}-01\end{array}$ & $\begin{array}{l}2.60 \mathrm{e}+00 \\
2.64 \mathrm{e}+00\end{array}$ & $\begin{array}{l}8.31 \mathrm{e}+00 \\
8.40 \mathrm{e}+00\end{array}$ & $\begin{array}{l}8.23 \mathrm{e}+00 \\
8.25 \mathrm{e}+00\end{array}$ & $\begin{array}{l}1.64 \mathrm{e}+00 \\
1.65 \mathrm{e}+00\end{array}$ \\
\hline$T / W$ & $\begin{array}{l}8.44 \mathrm{e}-02 \\
8.49 \mathrm{e}-02\end{array}$ & $1.10 \mathrm{e}-01$ & $\begin{array}{l}9.59 \mathrm{e}-02 \\
9.69 \mathrm{e}-02\end{array}$ & $\begin{array}{l}1.05 \mathrm{e}-01 \\
1.07 \mathrm{e}-01\end{array}$ & $\begin{array}{l}8.38 \mathrm{e}-02 \\
8.47 \mathrm{e}-02\end{array}$ & $\begin{array}{l}1.37 \mathrm{e}-01 \\
1.39 \mathrm{e}-01\end{array}$ & $1.36 \mathrm{e}-01$ & $\begin{array}{l}1.01 \mathrm{e}-01 \\
1.02 \mathrm{e}-01\end{array}$ \\
\hline$Z_{\mathrm{p}}$ & $2.46 \mathrm{e}-01$ & $\begin{array}{l}6.86 \mathrm{e}-01 \\
6.89 \mathrm{e}-01\end{array}$ & $\begin{array}{l}4.63 e-01 \\
4.66 \mathrm{e}-01\end{array}$ & $\begin{array}{l}7.44 \mathrm{e}-01 \\
7.49 \mathrm{e}-01\end{array}$ & $\begin{array}{l}1.90 \mathrm{e}-01 \\
1.93 \mathrm{e}-01\end{array}$ & $\begin{array}{l}8.26 \mathrm{e}-01 \\
8.31 \mathrm{e}-01\end{array}$ & $\begin{array}{l}8.18 \mathrm{e}-01 \\
8.21 \mathrm{e}-01\end{array}$ & $\begin{array}{l}2.82 \mathrm{e}-01 \\
2.84 \mathrm{e}-01\end{array}$ \\
\hline$Z_{\text {eq }}^{\mathrm{f}}$ & $-1.81 \mathrm{e}-01$ & $\begin{array}{l}-3.30 \mathrm{e}-01 \\
-3.31 \mathrm{e}-01\end{array}$ & $\begin{array}{l}-2.42 \mathrm{e}-01 \\
-2.43 \mathrm{e}-01\end{array}$ & $\begin{array}{l}-3.36 \mathrm{e}-01 \\
-3.38 \mathrm{e}-01\end{array}$ & $\begin{array}{l}-1.42 \mathrm{e}-01 \\
-1.43 \mathrm{e}-01\end{array}$ & $\begin{array}{l}-3.47 \mathrm{e}-01 \\
-3.49 \mathrm{e}-01\end{array}$ & $\begin{array}{l}-3.46 \mathrm{e}-01 \\
-3.47 \mathrm{e}-01\end{array}$ & $\begin{array}{l}-2.10 \mathrm{e}-01 \\
-2.11 \mathrm{e}-01\end{array}$ \\
\hline$Z_{\mathrm{eq}}^{\mathrm{b}}$ & $\begin{array}{l}6.96 \mathrm{e}-01 \\
6.98 \mathrm{e}-01\end{array}$ & $\begin{array}{l}1.94 \mathrm{e}+00 \\
1.96 \mathrm{e}+00\end{array}$ & $1.28 \mathrm{e}+00$ & $\begin{array}{l}2.10 \mathrm{e}+00 \\
2.12 \mathrm{e}+00\end{array}$ & $5.44 \mathrm{e}-01$ & $\begin{array}{l}2.43 \mathrm{e}+00 \\
2.46 \mathrm{e}+00\end{array}$ & $\begin{array}{l}2.41 \mathrm{e}+00 \\
2.42 \mathrm{e}+00\end{array}$ & $\begin{array}{l}8.11 \mathrm{e}-01 \\
8.12 \mathrm{e}-01\end{array}$ \\
\hline e & $\begin{array}{l}6.84 \mathrm{e}-01 \\
6.88 \mathrm{e}-01\end{array}$ & $\begin{array}{l}6.90 \mathrm{e}-01 \\
7.26 \mathrm{e}-01\end{array}$ & $\begin{array}{l}6.75 \mathrm{e}-01 \\
6.82 \mathrm{e}-01\end{array}$ & $\begin{array}{l}6.74 \mathrm{e}-01 \\
6.81 \mathrm{e}-01\end{array}$ & $\begin{array}{l}6.65 \mathrm{e}-01 \\
6.70 \mathrm{e}-01\end{array}$ & $\begin{array}{l}6.97 \mathrm{e}-01 \\
7.04 \mathrm{e}-01\end{array}$ & $\begin{array}{l}7.02 \mathrm{e}-01 \\
7.03 \mathrm{e}-01\end{array}$ & $\begin{array}{l}7.13 \mathrm{e}-01 \\
7.18 \mathrm{e}-01\end{array}$ \\
\hline
\end{tabular}

$$
\left.\left.\left.-\frac{1}{r \sin ^{2} \theta}\right)-\frac{3}{8} \mathrm{e}^{2 \psi-2 \nu} \partial \omega \partial \omega\right] \mathrm{e}^{\psi} r \mathrm{~d} r \mathrm{~d} \theta\right\}^{-1},
$$

with the abridged notation

$\partial \mu \partial \psi \equiv \frac{\partial \mu}{\partial r} \frac{\partial \psi}{\partial r}+\frac{1}{r^{2}} \frac{\partial \mu}{\partial \theta} \frac{\partial \psi}{\partial \theta}$

Then, we define:

$G R V 2 \equiv\left|1-\lambda_{2}\right|$,

GRV $3 \equiv\left|1-\lambda_{3}\right|$.

If the Einstein equations are satisfied, these quantities satisfy the following virial identities:

$G R V 2=0$,
$G R V 3=0$.

Since exact solutions for the stationary problems satisfy the above relations, we can choose GRV2 and GRV3 as the error indicators for numerically obtained solutions. Note that due to its three dimensional character, GRV3 gives a larger weight to the external layers of the star. GRV3 is a relativistic generalization of the Newtonian virial theorem Eq. (43).

In practice, however, it should be noted that the virial identities in the above form are not always close to the accuracy of numerical results. In particular, for GRV2 the 
Table 7. Rotating models with realistic EOSs (continued)

\begin{tabular}{|c|c|c|c|c|c|c|c|}
\hline Model & WFF(FPS)mr & WFF(NV)br & $\begin{array}{l}\text { WFF(NV)mr } \\
\text { (max. mass) }\end{array}$ & FPSbr & $\begin{array}{c}\text { FPSmr } \\
\text { (max. mass) }\end{array}$ & CLESbr & $\begin{array}{c}\text { CLESmr } \\
\text { (max. mass) }\end{array}$ \\
\hline EOS & WFF3+FPS & WFF $3+\mathrm{NV}$ & $\mathrm{WFF} 3+\mathrm{NV}$ & FPS & FPS & CLES & CLES \\
\hline$\varepsilon_{\mathrm{c}}\left[\mathrm{g} \mathrm{cm}^{-3}\right]$ & $2.70 \mathrm{e}+15$ & $9.70 \mathrm{e}+14$ & $2.71 \mathrm{e}+15$ & $1.02 \mathrm{e}+15$ & $2.91 \mathrm{e}+15$ & $1.81 \mathrm{e}+14$ & $4.20 \mathrm{e}+14$ \\
\hline$r_{\mathrm{p}} / r_{\mathrm{e}}$ & $5.65 \mathrm{e}-01$ & $6.30 \mathrm{e}-01$ & $5.65 \mathrm{e}-01$ & $6.40 \mathrm{e}-01$ & $5.68 \mathrm{e}-01$ & $5.90 \mathrm{e}-01$ & $5.31 \mathrm{e}-01$ \\
\hline$\Omega\left[10^{4} \mathrm{~s}^{-1}\right]$ & $1.15 \mathrm{e}+00$ & $\begin{array}{l}6.98 \mathrm{e}-01 \\
6.99 \mathrm{e}-01\end{array}$ & $1.15 \mathrm{e}+00$ & $6.97 \mathrm{e}-01$ & $1.18 \mathrm{e}+00$ & $3.63 \mathrm{e}-01$ & $\begin{array}{l}6.03 \mathrm{e}-01 \\
6.06 \mathrm{e}-01\end{array}$ \\
\hline$M\left[M_{\odot}\right]$ & $\begin{array}{l}2.19 \mathrm{e}+00 \\
2.20 \mathrm{e}+00\end{array}$ & $\begin{array}{l}1.41 \mathrm{e}+00 \\
1.42 \mathrm{e}+00\end{array}$ & $\begin{array}{l}2.19 \mathrm{e}+00 \\
2.20 \mathrm{e}+00\end{array}$ & $1.41 \mathrm{e}+00$ & $\begin{array}{l}2.12 \mathrm{e}+00 \\
2.13 \mathrm{e}+00\end{array}$ & $\begin{array}{l}1.41 \mathrm{e}+00 \\
1.42 \mathrm{e}+00\end{array}$ & $\begin{array}{l}6.64 \mathrm{e}+00 \\
6.71 \mathrm{e}+00\end{array}$ \\
\hline$M_{0}\left[M_{\odot}\right]$ & $2.55 \mathrm{e}+00$ & $\begin{array}{l}1.54 \mathrm{e}+00 \\
1.55 \mathrm{e}+00\end{array}$ & $2.55 \mathrm{e}+00$ & $1.54 \mathrm{e}+00$ & $2.46 \mathrm{e}+00$ & $1.50 \mathrm{e}+00$ & $\begin{array}{l}8.36 \mathrm{e}+00 \\
8.40 \mathrm{e}+00\end{array}$ \\
\hline$R_{\text {circ }}[\mathrm{km}]$ & $1.28 \mathrm{e}+01$ & $1.43 \mathrm{e}+01$ & $1.28 \mathrm{e}+01$ & $1.41 \mathrm{e}+01$ & $1.24 \mathrm{e}+01$ & $2.30 \mathrm{e}+01$ & $\begin{array}{l}2.86 \mathrm{e}+01 \\
2.87 \mathrm{e}+01\end{array}$ \\
\hline$c J / G M_{\odot}^{2}$ & $\begin{array}{l}3.22 \mathrm{e}+00 \\
3.23 \mathrm{e}+00\end{array}$ & $1.31 \mathrm{e}+00$ & $\begin{array}{l}3.21 \mathrm{e}+00 \\
3.22 \mathrm{e}+00\end{array}$ & $1.27 \mathrm{e}+00$ & $\begin{array}{l}2.96 \mathrm{e}+00 \\
2.97 \mathrm{e}+00\end{array}$ & $1.62 \mathrm{e}+00$ & $\begin{array}{l}3.61 \mathrm{e}+01 \\
3.64 \mathrm{e}+01\end{array}$ \\
\hline$I\left[10^{45} \mathrm{~g} \mathrm{~cm}^{2}\right]$ & $\begin{array}{l}2.45 \mathrm{e}+00 \\
2.46 \mathrm{e}+00\end{array}$ & $\begin{array}{l}1.64 \mathrm{e}+00 \\
1.65 \mathrm{e}+00\end{array}$ & $\begin{array}{l}2.44 \mathrm{e}+00 \\
2.46 \mathrm{e}+00\end{array}$ & $1.60 \mathrm{e}+00$ & $\begin{array}{l}2.20 \mathrm{e}+00 \\
2.21 \mathrm{e}+00\end{array}$ & $\begin{array}{l}3.92 \mathrm{e}+00 \\
3.93 \mathrm{e}+00\end{array}$ & $\begin{array}{l}5.25 \mathrm{e}+01 \\
5.30 \mathrm{e}+01\end{array}$ \\
\hline$T / W$ & $\begin{array}{l}1.22 \mathrm{e}-01 \\
1.23 \mathrm{e}-01\end{array}$ & $\begin{array}{l}1.00 \mathrm{e}-01 \\
1.01 \mathrm{e}-01\end{array}$ & $\begin{array}{l}1.22 \mathrm{e}-01 \\
1.23 \mathrm{e}-01\end{array}$ & $\begin{array}{l}9.70 \mathrm{e}-02 \\
9.76 \mathrm{e}-02\end{array}$ & $\begin{array}{l}1.17 \mathrm{e}-01 \\
1.18 \mathrm{e}-01\end{array}$ & $1.13 \mathrm{e}-01$ & $\begin{array}{l}1.92 \mathrm{e}-01 \\
1.97 \mathrm{e}-01\end{array}$ \\
\hline$Z_{\mathrm{p}}$ & $\begin{array}{l}7.63 e-01 \\
7.64 e-01\end{array}$ & $\begin{array}{l}2.81 \mathrm{e}-01 \\
2.83 \mathrm{e}-01\end{array}$ & $\begin{array}{l}7.62 \mathrm{e}-01 \\
7.64 \mathrm{e}-01\end{array}$ & $\begin{array}{l}2.81 \mathrm{e}-01 \\
2.82 \mathrm{e}-01\end{array}$ & $\begin{array}{l}7.47 \mathrm{e}-01 \\
7.55 \mathrm{e}-01\end{array}$ & $\begin{array}{l}1.60 \mathrm{e}-01 \\
1.61 \mathrm{e}-01\end{array}$ & $\begin{array}{l}1.46 \mathrm{e}+00 \\
1.49 \mathrm{e}+00\end{array}$ \\
\hline$Z_{\text {eq }}^{\mathrm{f}}$ & $\begin{array}{l}-3.39 \mathrm{e}-01 \\
-3.41 \mathrm{e}-01\end{array}$ & $\begin{array}{l}-2.10 \mathrm{e}-01 \\
-2.11 \mathrm{e}-01\end{array}$ & $\begin{array}{l}-3.38 \mathrm{e}-01 \\
-3.40 \mathrm{e}-01\end{array}$ & $\begin{array}{l}-2.05 \mathrm{e}-01 \\
-2.06 \mathrm{e}-01\end{array}$ & $\begin{array}{l}-3.37 \mathrm{e}-01 \\
-3.39 \mathrm{e}-01\end{array}$ & $\begin{array}{l}-1.92 \mathrm{e}-01 \\
-1.93 \mathrm{e}-01\end{array}$ & $\begin{array}{l}-4.02 \mathrm{e}-01 \\
-4.07 \mathrm{e}-01\end{array}$ \\
\hline$Z_{\text {eq }}^{\mathrm{b}}$ & $\begin{array}{l}2.19 \mathrm{e}+00 \\
2.21 \mathrm{e}+00\end{array}$ & $\begin{array}{l}8.10 \mathrm{e}-01 \\
8.12 \mathrm{e}-01\end{array}$ & $\begin{array}{l}2.18 \mathrm{e}+00 \\
2.20 \mathrm{e}+00\end{array}$ & $\begin{array}{l}8.03 \mathrm{e}-01 \\
8.05 \mathrm{e}-01\end{array}$ & $\begin{array}{l}2.15 \mathrm{e}+00 \\
2.17 \mathrm{e}+00\end{array}$ & $\begin{array}{l}5.24 \mathrm{e}-01 \\
5.25 \mathrm{e}-01\end{array}$ & $\begin{array}{l}5.89 \mathrm{e}+00 \\
6.14 \mathrm{e}+00\end{array}$ \\
\hline $\mathrm{e}$ & $\begin{array}{l}6.93 \mathrm{e}-01 \\
7.28 \mathrm{e}-01\end{array}$ & $\begin{array}{l}7.13 \mathrm{e}-01 \\
7.18 \mathrm{e}-01\end{array}$ & $\begin{array}{l}6.93 \mathrm{e}-01 \\
7.27 \mathrm{e}-01\end{array}$ & $\begin{array}{l}7.05 \mathrm{e}-01 \\
7.10 \mathrm{e}-01\end{array}$ & $\begin{array}{l}6.84 \mathrm{e}-01 \\
6.90 \mathrm{e}-01\end{array}$ & $\begin{array}{l}7.69 \mathrm{e}-01 \\
7.70 \mathrm{e}-01\end{array}$ & $\begin{array}{l}7.20 \mathrm{e}-01 \\
7.29 \mathrm{e}-01\end{array}$ \\
\hline
\end{tabular}

integration is done by integrating in the $\theta$ coordinate as seen from the definition of GRV2. If one does not use the $\theta$ coordinate for solving equilibrium structures, one needs to change variables and in that procedure accuracy may be lost and the resultant values may become worse than the "real" accuracy before the variable change.

Concerning the quantity GRV3, the metric potentials in the vacuum region contribute to the integral considerably. It implies that if only the finite regions are treated, as in the $\mathrm{KEH}(\mathrm{OR})$ code, a large portion of the integrand cannot be taken into account, However, the expressions for the GRV2 and GRV3 are not unique because we can do the integral by part and replace the second derivatives with the matter terms, using Einstein's equations. In this way, the contribution far away from the star becomes less important. In the $\mathrm{KEH}(\mathrm{OR})$ code, GRV2 and GRV3 are evaluated through

$$
\begin{aligned}
\lambda_{2}^{\prime} \equiv & 8 \pi \int_{0}^{r_{\max }} \int_{0}^{\pi}\left[p+(\varepsilon+p) \frac{v^{2}}{\left(1-v^{2}\right)}\right] \mathrm{e}^{2 \alpha} r \mathrm{~d} r \mathrm{~d} \theta \\
& \times\left\{\int _ { 0 } ^ { r _ { \operatorname { m a x } } } \int _ { 0 } ^ { \pi } \frac { 1 } { 2 } \left[\nu,_{r}^{2}+\frac{1}{r^{2}} \nu,,^{2}\right.\right. \\
& \left.-\nu\left(\nu, r r+\frac{1}{r} \nu,_{r}+\frac{1}{r^{2}} \nu, \theta \theta\right)\right] r \mathrm{~d} r \mathrm{~d} \theta \\
& -\frac{3}{4} \int_{0}^{r_{\max }} \int_{0}^{\pi} \omega\left[16 \pi \mathrm{e}^{2 \alpha} \frac{(\Omega-\omega)(\varepsilon+p)}{1-v^{2}}\right.
\end{aligned}
$$

$$
\begin{aligned}
& +\frac{1}{r} \omega,_{r}+\frac{\cot \theta}{r^{2}} \omega,_{\theta}+\omega,_{r}(\beta+\nu),_{r} \\
& \left.\left.+\frac{1}{r^{2}} \omega,_{\theta}(\beta+\nu),_{\theta}\right] \mathrm{e}^{2 \beta-2 \nu} r^{3} \sin ^{2} \theta \mathrm{d} r \mathrm{~d} \theta\right\}^{-1},
\end{aligned}
$$$$
\lambda_{3}^{\prime} \equiv 4 \pi \int_{0}^{\pi} \int_{0}^{r_{\max }}\left[3 p+(\varepsilon+p) \frac{v^{2}}{\left(1-v^{2}\right)}\right] \mathrm{e}^{2 \alpha+\beta} r^{2}
$$$$
\sin \theta \mathrm{d} r \mathrm{~d} \theta \times\left\{\int _ { 0 } ^ { \pi } \int _ { 0 } ^ { r _ { \operatorname { m a x } } } \left[-\nu\left(\partial^{2} \nu+\partial \nu \partial \beta\right)\right.\right.
$$$$
+\frac{1}{2} \alpha\left(\partial^{2} \beta+\partial \beta \partial \beta\right)-\frac{1}{2 r \tan \theta} \frac{\partial \alpha}{\partial r}\left(1-\mathrm{e}^{2 \alpha-2 \beta}\right)
$$$$
+\frac{1}{4 r \tan \theta} \frac{\partial \beta}{\partial r}\left(1-\mathrm{e}^{2 \alpha-2 \beta}\right)
$$$$
\left.-\frac{3}{8} \omega 16 \pi \mathrm{e}^{2 \beta-2 \nu} \frac{(\varepsilon+p)(\Omega-\omega)}{\left(1-v^{2}\right)} \mathrm{e}^{2 \beta-2 \nu} r^{2} \sin ^{2} \theta\right]
$$$$
\left.r^{2} \mathrm{e}^{\beta} \sin \theta \mathrm{d} r \mathrm{~d} \theta\right\}^{-1},
$$

with another abridged notation

$\partial^{2} \psi \equiv \frac{\partial^{2} \psi}{\partial r^{2}}+\frac{2}{r} \frac{\partial \psi}{\partial r}+\frac{1}{r^{2}} \frac{\partial^{2} \psi}{\partial \theta^{2}}+\frac{1}{r^{2} \tan \theta} \frac{\partial \psi}{\partial \theta}$,

and $r_{\max }$ is the distance of the truncated point beyond which actual numerical computations are not carried out in the $\mathrm{KEH}(\mathrm{OR})$ code. 
This rewrite does not break the mathematical identity. In a sense, it may make "identity" more trivial, and then what information the identities provide us becomes unclear. However, as far as the same expression of the identity in the same code is used, they can play a role as indicators of accuracy among models solved by each code.

\subsection{Tables of models and comparison}

The physical parameters of 45 models, computed in this comparison project, are displayed in Tables 1-7. All quantities are displayed to three significant figures. The lower and the upper bounds on each quantity, as obtained by comparing results from the three codes, are shown in the upper and lower rows for each corresponding quantity, respectively. It follows that quantities expressed in a single row can be regarded as "exact", to three significant figures.

Tables 1 and 2 display results for polytropes with in$\operatorname{dex} N=0.5,0.75,1.0$ and 1.5 . For each value of the polytropic index $N$ we compute the following models:

1. a spherical Newtonian model (denoted by the symbol $s n)$,

2. a rapidly rotating Newtonian model $(r n)$,

3. a nearly spherical, relativistic model $(s r)$,

4. the maximum mass model $(m r)$ and

5. a rapidly rotating relativistic model $(r r)$.

For the constant density case $(N=0)$, the spherical Newtonian and spherical relativistic models are displayed in Table 3 and the rapidly rotating Newtonian and rapidly rotating relativistic models in Table 4 . While all other models are specified by the central energy density $\varepsilon_{\mathrm{c}}$ and the ratio of the polar radius to the equatorial radius $r_{\mathrm{p}} / r_{\mathrm{e}}$, constant density models are specified by their central pressure and $r_{\mathrm{p}} / r_{\mathrm{e}}$.

For realistic equations of state, spherical models are shown in Table 5 and rotating models with $1.4 M_{\odot}(b r$ : binary pulsar mass and relativistic) and maximum mass models are shown in Tables 6 and 7. For the equation of state L, model L(L)mr uses four-point Lagrange interpolation, while $\mathrm{L}(\mathrm{H}) m r$ is the same model but computed using cubic Hermite interpolation.

From the tables displaying polytropic models one can see that the three codes have a good agreement on most quantities especially for soft polytropes. For stiff polytropes $(N<1.0)$ the agreement is somewhat smaller. For constant density models, the relative differences between the three codes become several percent. More sensitive quantities are the three redshifts and the eccentricity. It should be noted that redshift factors are local quantities which reflect the metric potentials at each point. This implies that local values of the metric potentials do not have the same agreement between different numerical codes, as integrated global quantities. For the eccentricity, one needs to compute the length along the surface of the star (see the definition of the eccentricity (42)). Since in the $\mathrm{KEH} \operatorname{codes} \mu=\cos \theta$ is used as the angular variable there arise numerical errors near the pole region, i.e. $\theta \simeq 0$. Thus, the differences in the values of the eccentricity also reflect this numerical error due to the choice of coordinates. This causes differences of up to a few percent in the eccentricity for rapidly rotating models. On the other hand, global quantities such as angular velocity, mass, radius and angular momentum agree quite well among results of different codes.

From Tables 5-7, similar tendencies can be observed for realistic equations of state. Models for the most EOSs, except EOS CLES, have a good agreement between the three codes, although the agreement is not as good as for polytropic models. By comparing models constructed with EOSs WFF(FPS) and WFF(NV), it is evident that the choice of the low density EOSs affects very little the structure of the star.

The main reason for the large differences in the constant density case is that the discontinuous density distribution is creating Gibbs phenomena near the surface and this affects all three codes. The reason for the smaller agreement for realistic EOSs, compared to polytropic EOSs, is that the necessary interpolation between tabulated data affects the accuracy with which the equation of hydrostationary equilibrium is satisfied. For EOS L, the choice of the interpolation scheme also affects the accuracy of the computed models, with the cubic Hermite scheme being a better choice compared to a four-point Lagrange interpolation (see the discussion in a later section). For the other realistic EOSs the choice of the interpolation scheme had a negligible effect on the accuracy of computed models.

\subsection{Detailed comparison}

In order to investigate further the differences among numerical results obtained by the three codes, we show more detailed results for models: N15sn, N15 $\mathrm{mr}$ and N05sn in Table 8, N05mr in Table 9, N00sn, N00rn and N00sr in Table 10, N00rr in Table 11, Gmr, Lsr and L(L)mr in Table 12, $\mathrm{L}(\mathrm{H}) m r$, WFF(FPS) $s r$ and WFF(FPS) $b r$ in Table 13 and CLESmr in Table 14. In these tables, values to eight figures for each physical quantity are shown, as well as the relative differences among results of the three codes.

The three relative differences diff1, diff2 and diff3 are defined as

$$
\begin{aligned}
\operatorname{diff} 1 & \equiv \frac{\mathrm{BGSM}-\mathrm{KEH}(\mathrm{OR})}{\mathrm{KEH}(\mathrm{OR})} \\
\operatorname{diff} 2 & \equiv \frac{\mathrm{KEH}(\mathrm{OR})-\mathrm{KEH}(\mathrm{SF})}{\mathrm{KEH}(\mathrm{SF})} \\
\operatorname{diff} 3 & \equiv \frac{\mathrm{KEH}(\mathrm{SF})-\mathrm{BGSM}}{\mathrm{BGSM}}
\end{aligned}
$$


Table 8. Detailed comparison of polytropic models

\begin{tabular}{|c|c|c|c|c|c|c|}
\hline Model & $\mathrm{KEH}(\mathrm{OR})$ & $\mathrm{KEH}(\mathrm{SF})$ & BGSM & diff1 & diff2 & diff3 \\
\hline Model & N15sn & & & & & \\
\hline $\bar{\varepsilon}_{\mathrm{c}}$ & $1.0000000 \mathrm{e}-09$ & $1.0000000 \mathrm{e}-09$ & $1.0000000 \mathrm{e}-09$ & & & \\
\hline$r_{\mathrm{p}} / r_{\mathrm{e}}$ & $9.6000000 \mathrm{e}-01$ & $9.6000000 \mathrm{e}-01$ & $9.6000000 \mathrm{e}-01$ & & & \\
\hline$\Omega$ & $6.5347464 \mathrm{e}-06$ & $6.5349925 \mathrm{e}-06$ & $6.5346091 \mathrm{e}-06$ & $-2 \mathrm{e}-5$ & $-4 \mathrm{e}-5$ & $6 e-5$ \\
\hline $\bar{M}$ & $9.7631159 \mathrm{e}-05$ & 9.763356 & $9.7634578 \mathrm{e}-05$ & $4 e-5$ & $-3 e-5$ & $-1 \mathrm{e}-5$ \\
\hline $\bar{M}_{0}$ & $9.7631237 \mathrm{e}-05$ & $9.7633644 \mathrm{e}-05$ & $9.7634657 \mathrm{e}-05$ & $4 e-5$ & $-3 e-5$ & $-1 \mathrm{e}-5$ \\
\hline $\bar{R}_{\text {circ }}$ & $5.2907355 \mathrm{e}+01$ & $5.2907099 \mathrm{e}+01$ & $5.2908476 \mathrm{e}+01$ & $2 \mathrm{e}-5$ & $5 e-6$ & $-3 \mathrm{e}-5$ \\
\hline $\bar{J}$ & $3.5985713 \mathrm{e}-07$ & $3.5988285 \mathrm{e}-07$ & $3.5988120 \mathrm{e}-07$ & $7 e-5$ & $-7 e-5$ & $5 e-6$ \\
\hline $\bar{I}$ & $5.5068262 \mathrm{e}-02$ & -02 & 02 & $9 e-5$ & $-3 e-5$ & $-5 \mathrm{e}-5$ \\
\hline$T / W$ & $7.4801796 \mathrm{e}-03$ & 7.48057 & $7.4802183 \mathrm{e}-03$ & $5 e-6$ & $-5 e-5$ & $5 e-5$ \\
\hline$Z_{\mathrm{p}}$ & $1.9104721 \mathrm{e}-06$ & $1.9105286 \mathrm{e}-06$ & $1.9104977 \mathrm{e}-06$ & $1 e-5$ & $-3 e-5$ & $2 \mathrm{e}-5$ \\
\hline$Z_{\text {eq }}^{\mathrm{f}}$ & $-3.4382698 \mathrm{e}-04$ & $-3.4383282 \mathrm{e}-04$ & $-3.4382701 \mathrm{e}-04$ & $9 \mathrm{e}-8$ & $-2 \mathrm{e}-5$ & $2 \mathrm{e}-5$ \\
\hline$Z_{\text {eq }}^{\mathrm{b}^{\mathrm{t}}}$ & $3.4764792 \mathrm{e}-04$ & $3.4765387 \mathrm{e}-04$ & $3.4764801 \mathrm{e}-4$ & $3 e-7$ & $-2 \mathrm{e}-5$ & $2 \mathrm{e}-5$ \\
\hline $\mathrm{e}$ & $2.7990046 \mathrm{e}-01$ & & $0 e-01$ & $9 e-4$ & $-1 \mathrm{e}-2$ & $1 e-2$ \\
\hline GRV2 & $1.0578017 \mathrm{e}-04$ & 6.365302 & $-9.0173678 \mathrm{e}-08$ & & & \\
\hline GRV3 & $-1.3712946 \mathrm{e}-05$ & 7.892486 & & & & \\
\hline Model & $\mathrm{N} 15 \mathrm{mr}$ & & & & & \\
\hline $\bar{\varepsilon}_{\mathrm{c}}$ & $0 \mathrm{e}-02$ & $.1000000 \mathrm{e}-02$ & $6.1000000 \mathrm{e}-02$ & & & \\
\hline$r_{\mathrm{p}} / r_{\mathrm{e}}$ & $2000000 \mathrm{e}-01$ & -01 & $e-01$ & & & \\
\hline $\bar{\Omega}$ & $1.1082917 \mathrm{e}-01$ & -01 & & $-3 e-4$ & $2 \mathrm{e}-4$ & $6 e-5$ \\
\hline $\bar{M}$ & $2.9099968 \mathrm{e}-$ & 2.9091 & & $-3 e-4$ & & $-1 \mathrm{e}-5$ \\
\hline $\bar{M}_{0}$ & $0433314 \mathrm{e}-01$ & -01 & $e-01$ & $1 e-4$ & $-1 \mathrm{e}-4$ & $-2 \mathrm{e}-5$ \\
\hline $\bar{R}_{\text {circ }}$ & $2.8538213 \mathrm{e}+00$ & +00 & $e+00$ & $3 e-5$ & $3 e-5$ & $-6 e-5$ \\
\hline $\bar{J}$ & $3.8794961 \mathrm{e}-02$ & & & $3 e-4$ & $-3 e-4$ & $-5 \mathrm{e}-5$ \\
\hline $\bar{I}$ & & & & $6 e-4$ & $-5 \mathrm{e}-4$ & $-1 e-4$ \\
\hline$T / W$ & $4.7633988 \mathrm{e}-02$ & 4.75086 & -02 & $-3 e-3$ & $3 e-3$ & $2 \mathrm{e}-5$ \\
\hline$Z_{\mathrm{p}}$ & $1.9429844 \mathrm{e}-01$ & 1.94785 & 1.94783 & $3 e-3$ & $-3 e-3$ & $1 e-5$ \\
\hline$Z_{\mathrm{eq}}^{\mathrm{f}}$ & $-2.2475260 \mathrm{e}-01$ & -2.24476 & -2.2448 & $-1 \mathrm{e}-3$ & $1 \mathrm{e}-3$ & $-2 \mathrm{e}-5$ \\
\hline$Z_{\mathrm{eq}}^{\mathrm{b}}$ & $6.2263043 \mathrm{e}-01$ & & & $1 e-3$ & $-1 \mathrm{e}-3$ & $4 e-6$ \\
\hline $\mathrm{e}^{\mathrm{i}}$ & & & & & & $-8 \mathrm{e}-4$ \\
\hline GRV2 & 5664 & & $-4.5069715 \mathrm{e}-07$ & & & \\
\hline GRV3 & $3198333 \mathrm{e}-05$ & & & & & \\
\hline Model & N05sn & & & & & \\
\hline $\bar{\varepsilon}_{\mathrm{c}}$ & $000000 e-03$ & & & & & \\
\hline$r_{\mathrm{p}} / r_{\mathrm{e}}$ & $000000 e-01$ & 9.8000000 & 9.80000 & & & \\
\hline $\bar{\Omega}$ & & & & & & \\
\hline $\bar{M}$ & & & & $-5 e-5$ & $-4 €$ & $8 \mathrm{e}-5$ \\
\hline $\bar{M}_{0}$ & $993012 \mathrm{e}-08$ & -08 & $989995 \mathrm{e}-08$ & $-5 \mathrm{e}-5$ & $-4 \mathrm{e}-5$ & $8 e-5$ \\
\hline $\bar{R}_{\text {circ }}$ & $3.0433836 \mathrm{e}-02$ & -02 & $432960 \mathrm{e}-02$ & $-3 e-5$ & $2 . e-6$ & $3 e-5$ \\
\hline $\bar{J}$ & $150934 \mathrm{e}-13$ & -13 & $88 \mathrm{e}-13$ & $-2 \mathrm{e}-3$ & $-2 e-4$ & $2 \mathrm{e}-3$ \\
\hline $\bar{I}$ & 1 & & & $-1 e-4$ & $-1 e-4$ & $2 \mathrm{e}-4$ \\
\hline$T / W$ & $5.1994036 \mathrm{e}-03$ & -03 & $e-03$ & $-3 e-3$ & $-2 \mathrm{e}-4$ & $4 e-3$ \\
\hline$Z_{\mathrm{p}}$ & $2.0985378 \mathrm{e}-06$ & $2.0986158 \mathrm{e}-06$ & $2.0984061 \mathrm{e}-06$ & $-6 e-5$ & $-3 e-5$ & $1 e-4$ \\
\hline$Z_{\mathrm{eq}}^{\mathrm{f}}$ & $-2.0851997 \mathrm{e}-04$ & $-2.0851108 \mathrm{e}-04$ & $-2.0812950 \mathrm{e}-04$ & $-2 \mathrm{e}-3$ & $4 \mathrm{e}-5$ & $2 \mathrm{e}-3$ \\
\hline$Z_{\mathrm{eq}}^{\mathrm{b}}$ & & $2.1270832 \mathrm{e}-04$ & $2.1232632 \mathrm{e}-04$ & $-2 \mathrm{e}-3$ & $4 e-5$ & $2 \mathrm{e}-3$ \\
\hline $\mathrm{e}^{1}$ & $1.9884365 \mathrm{e}-01$ & $2.0484010 \mathrm{e}-01$ & $1.9911770 \mathrm{e}-01$ & $1 e-3$ & $-3 e-2$ & $3 e-2$ \\
\hline GRV2 & $-7.8558660 \mathrm{e}-06$ & $2.5493102 \mathrm{e}-05$ & $-8.1720158 \mathrm{e}-06$ & & & \\
\hline GRV3 & $-1.1614383 \mathrm{e}-05$ & $9.1053508 \mathrm{e}-05$ & $-2.6069983 \mathrm{e}-06$ & & & \\
\hline
\end{tabular}

From Tables 8 and 9 , we see that the agreement between the $\mathrm{KEH}(\mathrm{SF})$ and BGSM codes for the rapidly rotating, relativistic polytropic models models N15 $\mathrm{mr}$ and N05mr is between $10^{-4}$ and $310^{-4}$ in all computed quantities (global and local), except for the eccentricity (due to reasons we explained in the previous section). This very good agreement shows that both the BGSM and KEH schemes (the latter when applying boundary conditions exactly at infinity) are suitable for the construction of highly accurate initial-data configurations of rapidly rotating relativistic stars, modeled as polytropes with typical index $N>0.5$.

From the same tables, we see that the agreement between the $\mathrm{KEH}(\mathrm{OR})$ and BGSM codes is similar to the 
Table 9. Detailed comparison of polytropic models (continued)

\begin{tabular}{ccccccc}
\hline Model & KEH(OR) & KEH(SF) & BGSM & diff1 & diff2 & diff3 \\
\hline Model & N05mr & & & & & \\
$\bar{\varepsilon}_{\mathrm{c}}$ & $1.0400000 \mathrm{e}+00$ & $1.0400000 \mathrm{e}+00$ & $1.0400000 \mathrm{e}+00$ & & & \\
$r_{\mathrm{p}} / r_{\mathrm{e}}$ & $5.5000000 \mathrm{e}-01$ & $5.5000000 \mathrm{e}-01$ & $5.5000156 \mathrm{e}-01$ & & & \\
$\bar{\Omega}$ & $9.8004159 \mathrm{e}-01$ & $9.7793068 \mathrm{e}-01$ & $9.7787248 \mathrm{e}-01$ & $-2 \mathrm{e}-3$ & $2 \mathrm{e}-3$ & $6 \mathrm{e}-5$ \\
$\bar{M}$ & $1.5376721 \mathrm{e}-01$ & $1.5302143 \mathrm{e}-01$ & $1.5301513 \mathrm{e}-01$ & $-5 \mathrm{e}-3$ & $5 \mathrm{e}-3$ & $4 \mathrm{e}-5$ \\
$\bar{M}_{0}$ & $1.8250033 \mathrm{e}-01$ & $1.8251809 \mathrm{e}-01$ & $1.8250776 \mathrm{e}-01$ & $4 \mathrm{e}-5$ & $-1 \mathrm{e}-4$ & $6 \mathrm{e}-5$ \\
$\bar{R}_{\text {circ }}$ & $5.3491240 \mathrm{e}-01$ & $5.3492344 \mathrm{e}-01$ & $5.3489664 \mathrm{e}-01$ & $-3 \mathrm{e}-5$ & $-2 \mathrm{e}-5$ & $5 \mathrm{e}-5$ \\
$\bar{J}$ & $1.7214828 \mathrm{e}-02$ & $1.7214474 \mathrm{e}-02$ & $1.7217664 \mathrm{e}-02$ & $2 \mathrm{e}-4$ & $2 \mathrm{e}-5$ & $-2 \mathrm{e}-4$ \\
$\bar{I}$ & $1.7565405 \mathrm{e}-02$ & $1.7602959 \mathrm{e}-02$ & $1.7607269 \mathrm{e}-02$ & $2 \mathrm{e}-3$ & $-2 \mathrm{e}-3$ & $-2 \mathrm{e}-4$ \\
$T / W$ & $1.4949334 \mathrm{e}-01$ & $1.4721775 \mathrm{e}-01$ & $1.4726559 \mathrm{e}-01$ & $-2 \mathrm{e}-2$ & $2 \mathrm{e}-2$ & $-3 \mathrm{e}-4$ \\
$Z_{\mathrm{p}}$ & $9.6404034 \mathrm{e}-01$ & $9.7533189 \mathrm{e}-01$ & $9.7519945 \mathrm{e}-01$ & $1 \mathrm{e}-2$ & $-1 \mathrm{e}-2$ & $1 \mathrm{e}-4$ \\
$Z_{\mathrm{eq}}^{\mathrm{f}}$ & $-3.6445066 \mathrm{e}-01$ & $-3.6225810 \mathrm{e}-01$ & $-3.6208301 \mathrm{e}-01$ & $-7 \mathrm{e}-3$ & $6 \mathrm{e}-3$ & $4 \mathrm{e}-4$ \\
$Z_{\mathrm{eq}}^{\mathrm{b}}$ & $2.9851556 \mathrm{e}+00$ & $3.0239590 \mathrm{e}+00$ & $3.0243455 \mathrm{e}+00$ & $1 \mathrm{e}-2$ & $-1 \mathrm{e}-2$ & $-1 \mathrm{e}-4$ \\
$\mathrm{e}$ & $6.9783608 \mathrm{e}-01$ & $6.9495486 \mathrm{e}-01$ & $7.0174527 \mathrm{e}-01$ & $6 \mathrm{e}-3$ & $4 \mathrm{e}-3$ & $-1 \mathrm{e}-2$ \\
GRV2 & $-2.9470467 \mathrm{e}-03$ & $3.5351133 \mathrm{e}-02$ & $-5.3131831 \mathrm{e}-05$ & & & \\
GRV3 & $-7.2928314 \mathrm{e}-02$ & $1.1781519 \mathrm{e}-04$ & $1.3585788 \mathrm{e}-04$ & & & \\
\hline \multicolumn{7}{c}{}
\end{tabular}

agreement between $\mathrm{KEH}(\mathrm{SF})$ and BGSM in the global quantities of model N15mr but to within $10^{-3}$ for the local quantities of this model. For the stiffer polytrope N05 $m r$ the agreement is $10^{-3}$ and $10^{-2}$ for global and local quantities, respectively. This difference in accuracy between $\mathrm{KEH}(\mathrm{SF})$ and $\mathrm{KEH}(\mathrm{OR})$ is expected, since in $\mathrm{KEH}(\mathrm{OR})$ boundary conditions are applied only approximately at a finite distance from the star. Considering how close to the star the domain of integration is truncated, the $\mathrm{KEH}(\mathrm{OR})$ code performs very well. This is explained as follows:

Since the integration is performed over only a finite region, the truncated part of the integral, $I_{\mathrm{tr}}$, can be expressed as

$I_{\mathrm{tr}}(\mathbf{r}) \equiv \int_{\text {out }} S\left(\mathbf{r}^{\prime}\right) G\left(\mathbf{r}, \mathbf{r}^{\prime}\right) \mathrm{d}^{3} \mathbf{r}^{\prime}$,

where $S$ and $G$ are the source term and the Green's function, respectively, and the subscript out denotes that the integration covers the region with $r \geq R_{\max }$. Here $R_{\max }$ is the maximum radius of the region of integration. Although the source terms of the integrals for the metric potentials in the $\mathrm{KEH}(\mathrm{OR})$ scheme contain both matter and the metric terms, only the metric terms contribute to the integral the outside of the star. Since we impose asymptotically flat conditions for the metric potentials, the radial dependence of the source term can be considered to be

$S \sim \frac{1}{r^{k}}$

where $k \geq 4$, because of the behavior of metric functions at large radii. Consequently, $I_{\mathrm{tr}}$ can be roughly expressed as

$$
\begin{aligned}
I_{\mathrm{tr}}(\mathbf{r}) & \sim \text { constant, } \quad\left(r<r_{\mathrm{e}}<R_{\max }\right) \\
& \sim \frac{1}{r^{k-2}} \ln \left(1-\frac{r}{R_{\max }}\right) . \quad\left(r_{\mathrm{e}}<r<R_{\max }\right) .
\end{aligned}
$$

From the above equation, we can see the following: First, for the stellar interior, i.e. $r<r_{\mathrm{e}}, I_{\mathrm{tr}} \sim$ constant, so that the relative error $I_{\mathrm{tr}} / I_{0}$ can be very small where $I_{0}$ is the exact value of the integral. Second, for the outer region of the stars, i.e. $r_{\mathrm{e}} \leq r \leq R_{\max }$, the value of $I_{\mathrm{tr}}$ becomes larger for larger values of $r$ because of the logarithmic term. Third, for the same region, i.e. $r_{\mathrm{e}} \leq r \leq R_{\max }$, if the source term depends weakly on the radial coordinate, i.e., for smaller values of $k$, the contribution of the truncated part becomes larger. This occurs for the potentials $\nu$ and $\omega$. However, for the metric functions $B$ and $\zeta$, since values of $k$ are large, the relative differences are rather small.

In the extreme case of constant density models (Tables 10 and 11), the three codes agree on the computed physical quantities typically only within a few percent and this is caused by the sharp density discontinuity at the surface of the star. The numerical schemes in this comparison assume that the density distribution is a smooth function of coordinates, thus, in the case of density discontinuities, this assumption is violated and Gibbs phenomena appear, resulting in low accuracy of the computed models.

From Tables 12-14 it follows that the agreement of the $\mathrm{KEH}(\mathrm{SF})$ code to the BGSM code is between $10^{-3}$ and $10^{-4}$ for realistic EOSs, except for EOS CLES, where the agreement is an order of magnitude smaller. $\mathrm{KEH}(\mathrm{OR})$ and BGSM agree on the realistic models within $10^{-2}$ and $10^{-3}$, i.e. similar to the agreement for the $N=0.5$ polytrope. The somewhat lesser agreement for realistic EOSs is due to the use of interpolation between the tabulated equation of state data (see the discussion next).

In Tables 8 to 14, we also display the virial quantities GRV2 and GRV3. In the ideal case, these should exactly vanish, so the smaller the values for GRV2 and GRV3 are, the better is the accuracy of the computed model. The opposite is not always true, i.e. in some models the computed values for GRV2 or GRV3 do not reflect an overall better agreement in physical quantities among the different codes. This indicates that the computation of 
Table 10. Detailed comparison of constant density models

\begin{tabular}{|c|c|c|c|c|c|}
\hline Model & KEH(OR) & $\mathrm{KEH}(\mathrm{SF})$ & BGSM & diff2 & diff3 \\
\hline Model & N00sn & & & & \\
\hline $\bar{\varepsilon}_{\mathrm{c}}$ & $1.0000000 \mathrm{e}+00$ & $1.0000000 \mathrm{e}+00$ & $1.0000000 \mathrm{e}+00$ & & \\
\hline $\bar{p}_{\mathrm{c}}$ & $1.0000000 \mathrm{e}-04$ & $1.0000000 \mathrm{e}-04$ & $1.0000000 \mathrm{e}-04$ & & \\
\hline$r_{\mathrm{p}} / r_{\mathrm{e}}$ & $1.0000000 \mathrm{e}+00$ & $1.0000000 \mathrm{e}+00$ & $1.0000000 \mathrm{e}+00$ & & \\
\hline $\bar{M}$ & $1.4049747 \mathrm{e}-06$ & $1.4079464 \mathrm{e}-06$ & $1.3811478 \mathrm{e}-06$ & $-2 \mathrm{e}-2-2 \mathrm{e}-3$ & $2 \mathrm{e}-2$ \\
\hline $\bar{M}_{0}$ & $1.4051472 \mathrm{e}-06$ & $1.4081229 \mathrm{e}-06$ & $1.3813135 \mathrm{e}-06$ & $-2 \mathrm{e}-2-2 \mathrm{e}-3$ & $2 e-2$ \\
\hline $\bar{R}_{\text {circ }}$ & $6.9250048 \mathrm{e}-03$ & $6.9085570 \mathrm{e}-03$ & $6.9085014 \mathrm{e}-03$ & $-2 \mathrm{e}-3 \quad 2 \mathrm{e}-3$ & $8 \mathrm{e}-6$ \\
\hline$Z_{\mathrm{p}}$ & $2.0197813 \mathrm{e}-04$ & $2.0386139 \mathrm{e}-04$ & $1.9998000 \mathrm{e}-04$ & $-1 e-2-9 e-3$ & $2 \mathrm{e}-2$ \\
\hline$Z_{\mathrm{eq}}^{\mathrm{f}}$ & $2.0197813 \mathrm{e}-04$ & $2.0386139 \mathrm{e}-04$ & $1.9998000 \mathrm{e}-04$ & $-1 \mathrm{e}-2-9 \mathrm{e}-3$ & $2 \mathrm{e}-2$ \\
\hline$Z_{\mathrm{eq}}^{\mathrm{b}}$ & $2.0197813 \mathrm{e}-04$ & $2.0386139 \mathrm{e}-04$ & $1.9998000 \mathrm{e}-04$ & $-1 e-2-9 e-3$ & $2 e-2$ \\
\hline GRV2 & $8.5024396 \mathrm{e}-03$ & $2.5005801 \mathrm{e}-02$ & $1.9992785 \mathrm{e}-10$ & & \\
\hline GRV3 & $1.1669659 \mathrm{e}-02$ & $3.1034530 \mathrm{e}-02$ & $2.4994651 \mathrm{e}-10$ & & \\
\hline Model & N00rn & & & & \\
\hline $\bar{\varepsilon}_{\mathrm{c}}$ & $1.0000000 \mathrm{e}+00$ & $1.0000000 \mathrm{e}+00$ & $1.0000000 \mathrm{e}+00$ & & \\
\hline $\bar{p}_{\mathrm{c}}$ & $1.0000000 \mathrm{e}-04$ & $1.0000000 \mathrm{e}-04$ & $1.0000000 \mathrm{e}-04$ & & \\
\hline$r_{\mathrm{p}} / r_{\mathrm{e}}$ & $6.5000000 \mathrm{e}-01$ & $6.5000000 \mathrm{e}-01$ & $6.4996883 \mathrm{e}-01$ & $-5 e-5$ & $5 e-5$ \\
\hline $\bar{\Omega}$ & $1.0038565 \mathrm{e}+00$ & $1.0145815 \mathrm{e}+00$ & $1.0154202 \mathrm{e}+00$ & $1 e-2-1 e-2$ & $-8 \mathrm{e}-4$ \\
\hline $\bar{M}$ & $1.8558447 \mathrm{e}+00$ & 1.84668 & $1.8532465 \mathrm{e}+00$ & $-1 e-3 \quad 5 e-3$ & $-4 e-3$ \\
\hline $\bar{M}$ & $2.0497584 \mathrm{e}-06$ & 2.06377 & $2.0569322 \mathrm{e}-06$ & $4 e-3-7 e-3$ & $3 e-3$ \\
\hline $\bar{M}_{0}$ & $2.0500389 \mathrm{e}-06$ & $2.0640573 \mathrm{e}-06$ & $2.0572047 \mathrm{e}-06$ & $-7 e-3$ & $3 e-3$ \\
\hline $\bar{R}_{\text {circ }}$ & $9.0583317 \mathrm{e}-03$ & $9.1109677 \mathrm{e}-03$ & $9.1096124 \mathrm{e}-03$ & $-6 e-3$ & $2 e-4$ \\
\hline $\bar{J}$ & $6.7616039 \mathrm{e}-11$ & 6.96685 & $6.9165592 \mathrm{e}-11$ & $-3 e-2$ & $7 e-3$ \\
\hline $\bar{I}$ & $6.7356280 \mathrm{e}-11$ & 6.86672 & $6.8115244 \mathrm{e}-11$ & $-2 \mathrm{e}-2$ & $8 e-3$ \\
\hline$T / W$ & $1.0792359 \mathrm{e}-01$ & $1.1089017 \mathrm{e}$ & $416842 \mathrm{e}-01$ & $-3 e-2$ & $-3 e-2$ \\
\hline$Z_{\mathrm{p}}$ & $2.8331355 \mathrm{e}-04$ & $2.8580524 \mathrm{e}-04$ & $2.8505153 \mathrm{e}-04$ & $-9 \mathrm{e}-3$ & $3 e-3$ \\
\hline$Z_{\mathrm{eq}}^{\mathrm{f}}$ & $-8.8147112 \mathrm{e}-03$ & $-8.9620804 \mathrm{e}-03$ & $-8.9698802 \mathrm{e}-03$ & $-2 \mathrm{e}-2$ & $-9 \mathrm{e}-4$ \\
\hline$Z_{\text {eq }}^{\mathrm{b}^{\mathrm{t}}}$ & $9.3813731 \mathrm{e}-03$ & $9.5337271 \mathrm{e}-03$ & $9.5400451 \mathrm{e}-03$ & $-2 \mathrm{e}-2$ & $-7 e-4$ \\
\hline $\mathrm{e}$ & $7.5985797 \mathrm{e}-01$ & $7.6083298 \mathrm{e}-01$ & $7.5926925 \mathrm{e}-01$ & $-8 \mathrm{e}-4-1$ & $2 \mathrm{e}-3$ \\
\hline GRV2 & $-1.0486413 \mathrm{e}-03$ & $4.0913020 \mathrm{e}-02$ & $1.5297023 \mathrm{e}-03$ & & \\
\hline GRV3 & $-1.9636423 \mathrm{e}-03$ & $6.3495351 \mathrm{e}-04$ & $1.8732802 \mathrm{e}-03$ & & \\
\hline Model & N00sr & & & & \\
\hline $\bar{\varepsilon}_{\mathrm{c}}$ & $1.0000000 \mathrm{e}+00$ & $1.0000000 \mathrm{e}+00$ & $1.0000000 \mathrm{e}+00$ & & \\
\hline $\bar{p}_{\mathrm{c}}$ & $1.0000000 \mathrm{e}+00$ & $1.0000000 \mathrm{e}+00$ & $1.0000000 \mathrm{e}+00$ & & \\
\hline$r_{\mathrm{p}} / r_{\mathrm{e}}$ & $1.0000000 \mathrm{e}+00$ & $1.0000000 \mathrm{e}+00$ & $1.0000000 \mathrm{e}+00$ & & \\
\hline $\bar{M}$ & $1.1404379 \mathrm{e}-01$ & $1.1257086 \mathrm{e}-01$ & $1.1220252 \mathrm{e}-01$ & $-2 \mathrm{e}-2 \quad 1 \mathrm{e}-2$ & $3 e-3$ \\
\hline $\bar{M}_{0}$ & $1.5912882 \mathrm{e}-01$ & $1.6139149 \mathrm{e}-01$ & $1.5914795 \mathrm{e}-01$ & $1 e-4-1 e-2$ & $1 e-2$ \\
\hline $\bar{R}_{\text {circ }}$ & $3.0003488 \mathrm{e}-01$ & $2.9923839 \mathrm{e}-01$ & $2.9920671 \mathrm{e}-01$ & $-3 e-3 \quad 3 e-3$ & $1 e-4$ \\
\hline$Z_{\mathrm{p}}$ & $9.7107121 \mathrm{e}-01$ & $1.0138916 \mathrm{e}+00$ & $1.0000000 \mathrm{e}+00$ & $3 e-2-4 e-2$ & $1 e-2$ \\
\hline$Z_{\mathrm{eq}}^{\mathrm{f}}$ & $9.7107121 \mathrm{e}-01$ & $1.0138916 \mathrm{e}+00$ & $1.0000000 \mathrm{e}+00$ & $-4 \mathrm{e}-2$ & $1 e-2$ \\
\hline$Z_{\mathrm{eq}}^{\mathrm{b}}$ & $9.7107121 \mathrm{e}-01$ & $1.0138916 \mathrm{e}+00$ & $1.0000000 \mathrm{e}+00$ & $3 e-2-4 e-2$ & $1 e-2$ \\
\hline GRV2 & $-4.2458406 \mathrm{e}-03$ & $8.0302649 \mathrm{e}-03$ & $1.2600343 \mathrm{e}-10$ & & \\
\hline GRV3 & $3.4088425 \mathrm{e}-02$ & $7.3549407 \mathrm{e}-03$ & $1.3790569 \mathrm{e}-10$ & & \\
\hline
\end{tabular}

GRV2 and GRV3 may itself be prone to numerical error. This seems to be the case for GRV2 in rapidly rotating models computed with the $\mathrm{KEH}(\mathrm{SF})$ code, where one first has to interpolate data between $\cos \theta$ and $\theta$ grids to be able to compute GRV2. Note that the displayed values of the two virial quantities for the $\mathrm{KEH}(\mathrm{OR})$ code correspond to the modified virial identities (61) and (62) and not to the original identities. The computation of GRV3 for the $\mathrm{KEH}(\mathrm{OR})$ code is affected significantly by the truncation of the domain of integration.

\section{Discussion and conclusion}

\subsection{Discussion}

\subsubsection{Metric potentials}

As redshift factors differ by about $10 \%$ for the constant density, relativistic model N00rr between the three codes (while the agreement global quantities is within a few \%) we compare directly the local values of metric potentials for several models. Figures 1 to 16 show the four metric potentials (upper panel) and the relative differences in them between BGSM and $\mathrm{KEH}(\mathrm{OR})$ (middle panel) and 
Table 11. Detailed comparison of constant density models (continued)

\begin{tabular}{ccccccc}
\hline Model & KEH(OR) & KEH(SF) & BGSM & diff1 & diff2 & diff3 \\
\hline Model & N00rr & & & & & \\
$\bar{\varepsilon}_{\mathrm{c}}$ & $1.0000000 \mathrm{e}+00$ & $1.0000000 \mathrm{e}+00$ & $1.0000000 \mathrm{e}+00$ & & & \\
$\bar{p}_{\mathrm{c}}$ & $1.0000000 \mathrm{e}+00$ & $1.0000000 \mathrm{e}+00$ & $1.0000000 \mathrm{e}+00$ & & & \\
$r_{\mathrm{p}} / r_{\mathrm{e}}$ & $7.0000000 \mathrm{e}-01$ & $7.0000000 \mathrm{e}-01$ & $7.0075459 \mathrm{e}-01$ & $1 \mathrm{e}-3$ & & $-1 \mathrm{e}-3$ \\
$\bar{\Omega}$ & $1.3961457 \mathrm{e}+00$ & $1.4071531 \mathrm{e}+00$ & $1.3980528 \mathrm{e}+00$ & $1 \mathrm{e}-3$ & $-8 \mathrm{e}-3$ & $7 \mathrm{e}-3$ \\
$\bar{M}$ & $1.8323492 \mathrm{e}+00$ & $1.8121685 \mathrm{e}+00$ & $1.8158693 \mathrm{e}+00$ & $-9 \mathrm{e}-3$ & $1 \mathrm{e}-2$ & $-2 \mathrm{e}-3$ \\
$\bar{M}$ & $1.3889820 \mathrm{e}-01$ & $1.3605365 \mathrm{e}-01$ & $1.3462398 \mathrm{e}-01$ & $-3 \mathrm{e}-2$ & $2 \mathrm{e}-2$ & $1 \mathrm{e}-2$ \\
$\bar{M}_{0}$ & $1.8665283 \mathrm{e}-01$ & $1.8693714 \mathrm{e}-01$ & $1.8377945 \mathrm{e}-01$ & $-2 \mathrm{e}-2$ & $-2 \mathrm{e}-3$ & $2 \mathrm{e}-2$ \\
$\bar{R}_{\text {circ }}$ & $3.4513863 \mathrm{e}-01$ & $3.4566026 \mathrm{e}-01$ & $3.4455898 \mathrm{e}-01$ & $-2 \mathrm{e}-3$ & $-2 \mathrm{e}-3$ & $3 \mathrm{e}-3$ \\
$\bar{J}$ & $1.3838952 \mathrm{e}-02$ & $1.4064876 \mathrm{e}-02$ & $1.3733153 \mathrm{e}-02$ & $-8 \mathrm{e}-3$ & $-2 \mathrm{e}-2$ & $2 \mathrm{e}-2$ \\
$\bar{I}$ & $9.9122546 \mathrm{e}-03$ & $9.9952703 \mathrm{e}-03$ & $9.8230574 \mathrm{e}-03$ & $-9 \mathrm{e}-3$ & $-8 \mathrm{e}-3$ & $2 \mathrm{e}-2$ \\
$T / W$ & $1.6825846 \mathrm{e}-01$ & $1.6281418 \mathrm{e}-01$ & $1.6338670 \mathrm{e}-01$ & $-3 \mathrm{e}-2$ & $3 \mathrm{e}-2$ & $-4 \mathrm{e}-3$ \\
$Z_{\mathrm{p}}$ & $1.6030994 \mathrm{e}+00$ & $1.7071394 \mathrm{e}+00$ & $1.6720522 \mathrm{e}+00$ & $4 \mathrm{e}-2$ & $-6 \mathrm{e}-2$ & $2 \mathrm{e}-2$ \\
$Z_{\mathrm{eq}}^{\mathrm{f}}$ & $-1.5970481 \mathrm{e}-01$ & $-1.5951673 \mathrm{e}-01$ & $-1.5541165 \mathrm{e}-01$ & $-3 \mathrm{e}-2$ & $1 \mathrm{e}-3$ & $3 \mathrm{e}-2$ \\
$Z_{\mathrm{eq}}^{\mathrm{b}}$ & $9.4121600 \mathrm{e}+00$ & $1.1342393 \mathrm{e}+01$ & $1.0436972 \mathrm{e}+01$ & $1 \mathrm{e}-1$ & $-2 \mathrm{e}-1$ & $9 \mathrm{e}-2$ \\
$\mathrm{e}$ & $7.0676794 \mathrm{e}-01$ & $7.1111349 \mathrm{e}-01$ & $7.0812060 \mathrm{e}-01$ & $2 \mathrm{e}-3$ & $-6 \mathrm{e}-3$ & $4 \mathrm{e}-3$ \\
GRV2 & $-1.5336628 \mathrm{e}-02$ & $4.5755715 \mathrm{e}-02$ & $1.7034437 \mathrm{e}-03$ & & & \\
GRV3 & $-1.0930542 \mathrm{e}-01$ & $9.5032627 \mathrm{e}-04$ & $4.2345737 \mathrm{e}-03$ & & & \\
\hline
\end{tabular}
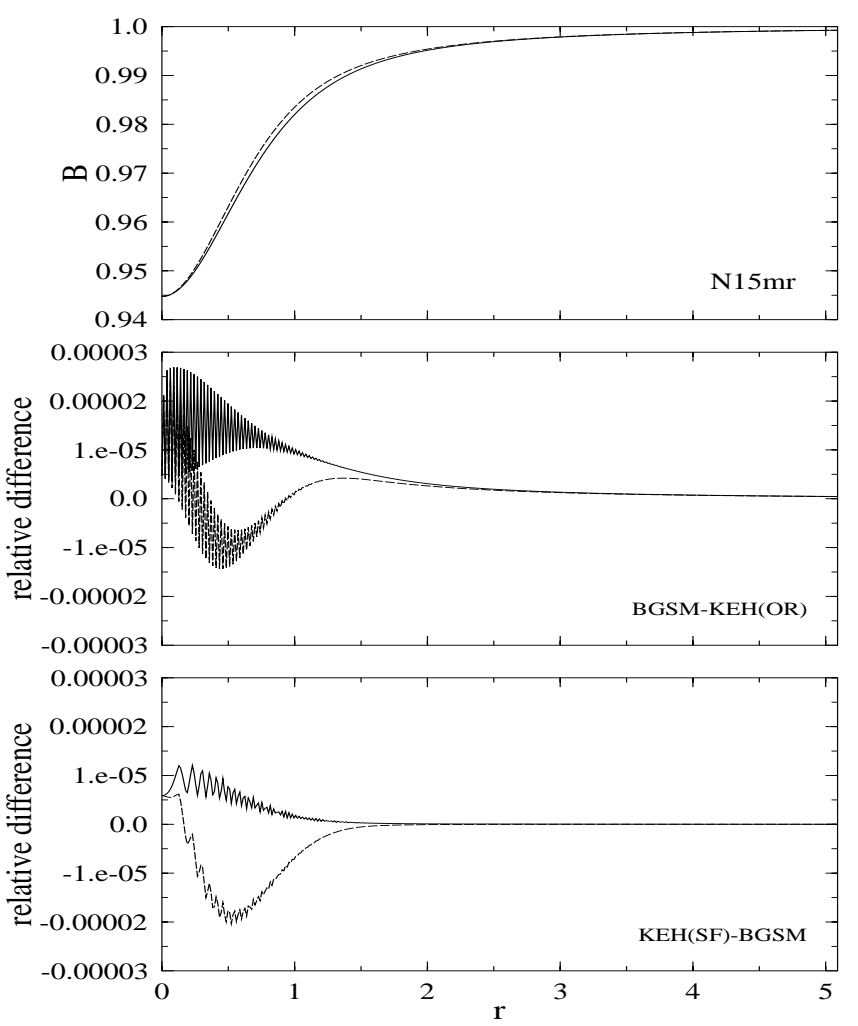

Fig. 1. Metric potential $B$ as a function of coordinate radius for model N15mr (upper panel). Relative difference of $B$ for the same model constructed with the BGSM and $\mathrm{KEH}(\mathrm{OR})$ codes (middle panel) and with the $\mathrm{KEH}(\mathrm{SF})$ and BGSM codes (lower panel). The solid graph corresponds to $\theta=\pi / 2$ (equatorial plane) and the dashed line to $\theta=0$ (axis of rotation). The largest value of $r$ displayed, corresponds to twice the coordinate radius of the surface of the star in the equatorial plane
$\mathrm{KEH}(\mathrm{SF})$ and BGSM (lower panel) for the models N05mr, $\mathrm{N} 15 m r, \mathrm{~L}(\mathrm{~L}) m r$ and WFF(FPS) $b r$. The metric potentials are graphed against the coordinate $r$ in the equatorial plane $(\theta=\pi / 2$, solid line) and along the axis of rotation $(\theta=0$, dashed line). The range of the coordinate $r$ is the twice the equatorial radius of the star.

In general, the agreement in the local values of the metric potentials reflects the agreement in the computed physical parameters of models. In these graphs, several significant behaviors can be pointed out: First, there are high frequency and small amplitude oscillations at the inner part of the stars for all models. Second, the differences between the results of $\mathrm{KEH}(\mathrm{OR})$ and those of the other two codes are growing outside the stars as $r$ increases. Third, although the differences between the $\mathrm{KEH}(\mathrm{SF})$ and BGSM codes are very small for models N05mr, N15mr and WFF(FPS) $b r$, there appear larger differences for the stiff model L(L) $m r$. Fourth, there appears a larger amplitude oscillation in the metric potential $\omega$ on the axis of rotation, close to the surface.

The first behavior is due to the integration scheme of the KEH code, i.e. the Simpson scheme. In general, the Simpson scheme gives results with higher precision, compared with those obtained by the trapezoidal scheme. However, in the KEH scheme, the integrands contain nonsmooth functions with respect to the radial coordinate, because of the nature of the Green's functions. The nonuniform distribution of the weight factor in Simpson's scheme for nonsmooth functions results in oscillating behaviors with very small amplitudes, which cannot be noticed in the behavior the integrated quantities.

The second behavior in the original KEH code is caused by the "truncation" of the domain of integration 
Table 12. Detailed comparison of models with realistic EOSs

\begin{tabular}{|c|c|c|c|c|c|c|}
\hline Model & KEH(OR) & $\mathrm{KEH}(\mathrm{SF})$ & BGSM & diff1 & diff2 & diff3 \\
\hline Model & $\mathrm{Gmr}$ & & & & & \\
\hline$\varepsilon_{\mathrm{c}}\left[\mathrm{g} \mathrm{cm}^{-3}\right]$ & $5.5828200 \mathrm{e}+15$ & $5.5828200 \mathrm{e}+15$ & $5.5828192 \mathrm{e}+15$ & & & \\
\hline$r_{\mathrm{p}} / r_{\mathrm{e}}$ & $5.7654000 \mathrm{e}-01$ & $5.7654000 \mathrm{e}-01$ & $5.7654388 \mathrm{e}-01$ & & & \\
\hline$\Omega\left[10^{4} \mathrm{~s}^{-1}\right]$ & $1.5778207 \mathrm{e}+00$ & $1.5729910 \mathrm{e}+00$ & $1.5731590 \mathrm{e}+00$ & $-3 e-3$ & $3 e-3$ & $-1 e-4$ \\
\hline$M\left[M_{\odot}\right]$ & $1.5705842 \mathrm{e}+00$ & $1.5670837 \mathrm{e}+00$ & $1.5652708 \mathrm{e}+00$ & $-3 e-3$ & $2 \mathrm{e}-3$ & $1 e-3$ \\
\hline$M_{0}\left[M_{\odot}\right]$ & $1.7915598 \mathrm{e}+00$ & $1.7930714 \mathrm{e}+00$ & $1.7913915 \mathrm{e}+00$ & $-9 e-5$ & $-8 \mathrm{e}-4$ & $9 e-4$ \\
\hline$R_{\text {circ }}[\mathrm{km}]$ & $9.2371483 \mathrm{e}+00$ & $9.2454157 \mathrm{e}+00$ & $9.2471834 \mathrm{e}+00$ & $1 e-3$ & $-9 \mathrm{e}-4$ & $-2 \mathrm{e}-4$ \\
\hline$c J / G M_{\odot}^{2}$ & $1.5360080 \mathrm{e}+00$ & $1.5378972 \mathrm{e}+00$ & $1.5369015 \mathrm{e}+00$ & $6 e-4$ & $-1 \mathrm{e}-3$ & $7 e-4$ \\
\hline$I\left[10^{45} \mathrm{~g} \mathrm{~cm}^{2}\right]$ & $8.5553062 \mathrm{e}-01$ & $8.5923863 \mathrm{e}-01$ & $8.5859068 \mathrm{e}-01$ & $4 e-3$ & $-4 e-3$ & $8 \mathrm{e}-4$ \\
\hline$T / W$ & $1.0661337 \mathrm{e}-01$ & & & $-1 e-2$ & $1 e-2$ & $-9 e-4$ \\
\hline$Z_{\mathrm{p}}$ & $7.4862286 \mathrm{e}-01$ & $7.4468655 \mathrm{e}-01$ & $7.4429886 \mathrm{e}-01$ & $-6 e-3$ & $5 e-3$ & $5 \mathrm{e}-4$ \\
\hline$Z_{\mathrm{eq}}^{\mathrm{f}}$ & $-3.3808636 \mathrm{e}-01$ & $-3.3618145 \mathrm{e}-01$ & $-3.3645247 \mathrm{e}-01$ & $-5 \mathrm{e}-3$ & $6 e-3$ & $-8 \mathrm{e}-4$ \\
\hline$Z_{\mathrm{eq}}^{\mathrm{b}}$ & $2.1006841 \mathrm{e}+00$ & $2.1151171 \mathrm{e}+00$ & $2.1140605 \mathrm{e}+00$ & $6 e-3$ & $-7 \mathrm{e}-3$ & $5 e-4$ \\
\hline $\mathrm{e}$ & 01 & & & $9 e-3$ & $-1 e-2$ & $2 e-3$ \\
\hline GRV2 & $534 e-03$ & -02 & $-3.6339736 \mathrm{e}-04$ & & & \\
\hline GRV3 & $-3.2386612 \mathrm{e}-02$ & & & & & \\
\hline Model & Lsr & & & & & \\
\hline$\varepsilon_{\mathrm{c}}\left[\mathrm{g} \mathrm{cm}^{-3}\right]$ & $.2995000 \mathrm{e}+14$ & $\mathrm{e}+14$ & $1 e+14$ & & & \\
\hline$r_{\mathrm{p}} / r_{\mathrm{e}}$ & 00 & & & & & \\
\hline$M\left[M_{\odot}\right]$ & $4097969 \mathrm{e}+00$ & 00 & -00 & $1 e-4$ & $2 e-2$ & $-2 \mathrm{e}-2$ \\
\hline$M_{0}\left[M_{\odot}\right]$ & $5235811 \mathrm{e}+00$ & & & $1 e-3$ & $2 \mathrm{e}-2$ & $-2 \mathrm{e}-2$ \\
\hline$R_{\text {circ }}[\mathrm{km}]$ & $1.4784119 \mathrm{e}+01$ & 1.4819 & 1.47784 & $-4 e-4$ & $-2 \mathrm{e}-3$ & $3 e-3$ \\
\hline$Z_{\mathrm{p}}$ & $1.7822863 \mathrm{e}-01$ & 1.7557 & 1.797417 & $9 e-3$ & $2 \mathrm{e}-2$ & $-2 \mathrm{e}-2$ \\
\hline$Z_{\mathrm{eq}}^{\mathrm{f}}$ & $1.7829440 \mathrm{e}-01$ & $1.7557838 \mathrm{e}-01$ & $1.7974175 \mathrm{e}-01$ & $8 \mathrm{e}-3$ & $2 \mathrm{e}-2$ & $-2 \mathrm{e}-2$ \\
\hline$Z_{\mathrm{eq}}^{\mathrm{b}}$ & $1.7829440 \mathrm{e}-01$ & & & $8 e-3$ & $2 \mathrm{e}-2$ & $-2 \mathrm{e}-2$ \\
\hline GRV2 & -3.9884 & -02 & 3.18 & & & \\
\hline GRV3 & $4064437 \mathrm{e}-$ & & & & & \\
\hline Model & $\mathrm{L}(\mathrm{L}) \mathrm{mr}$ & & & & & \\
\hline$\varepsilon_{\mathrm{c}}\left[\mathrm{g} \mathrm{cm}^{-3}\right]$ & $2022600 \mathrm{e}+15$ & & & & & \\
\hline$r_{\mathrm{p}} / r_{\mathrm{e}}$ & $5300000 e-01$ & $5.5300000 \mathrm{e}-01$ & $5.5300318 \mathrm{e}-01$ & & & \\
\hline$\Omega\left[10^{4} \mathrm{~s}^{-1}\right]$ & & & & $-2 \mathrm{e}-3$ & $1 e-3$ & $9 e-5$ \\
\hline$M\left[M_{\odot}\right]$ & -00 & & & $-3 e-3$ & $e-3$ & $-3 e-3$ \\
\hline$M_{0}\left[M_{\odot}\right]$ & $9164459 \mathrm{e}+$ & & & $1 e-3$ & $5 e-3$ & $-6 e-3$ \\
\hline$R_{\text {circ }}[\mathrm{km}]$ & $8472119 \mathrm{e}+01$ & $1.8460489 \mathrm{e}+01$ & $8475482 \mathrm{e}+01$ & $2 e-4$ & $6 e-4$ & $-8 \mathrm{e}-4$ \\
\hline$c J / G M_{\odot}^{2}$ & & $7.7201999 \mathrm{e}+00$ & $8026429 \mathrm{e}+00$ & $3 e-3$ & $8 \mathrm{e}-3$ & $-1 e-2$ \\
\hline$I\left[10^{45} \mathrm{~g} \mathrm{~cm}^{2}\right]$ & & & & $4 e-3$ & $6 e-3$ & $-1 e-2$ \\
\hline$T / W$ & 01 & & $8 \mathrm{e}-01$ & $-1 e-2$ & $2 \mathrm{e}-2$ & $-6 e-3$ \\
\hline$Z_{\mathrm{p}}$ & $8.2621901 \mathrm{e}-01$ & $8.2779278 \mathrm{e}-01$ & $8.3079056 \mathrm{e}-01$ & $6 e-3$ & $-2 \mathrm{e}-3$ & $-4 \mathrm{e}-3$ \\
\hline$Z_{\text {eq }}^{\mathrm{f}}$ & $-3.4862304 \mathrm{e}-01$ & $-3.4713361 \mathrm{e}-01$ & $-3.4699020 \mathrm{e}-01$ & $-5 \mathrm{e}-3$ & $4 e-3$ & $4 e-4$ \\
\hline$Z_{\text {eq }}^{\mathrm{b}}$ & & & & & $-6 e-3$ & $-5 e-3$ \\
\hline $\mathrm{e}$ & $1 e-01$ & $e-01$ & $7.0273768 \mathrm{e}-01$ & $8 e-3$ & $-9 e-3$ & $1 e-3$ \\
\hline GRV2 & $4730085 \mathrm{e}-04$ & $3.4941975 \mathrm{e}-02$ & $-2.2424315 \mathrm{e}-03$ & & & \\
\hline GRV3 & $-4.0789432 \mathrm{e}-02$ & $2.4558001 \mathrm{e}-04$ & $-2.1163857 \mathrm{e}-03$ & & & \\
\hline
\end{tabular}

at a finite distance from the star, instead of integrating over the whole space.

The large differences in the metric potentials between $\mathrm{KEH}(\mathrm{SF})$ and BGSM for EOS L, could be accounted to the stiffness of the equation of state, but the differences between $\mathrm{KEH}(\mathrm{OR})$ and BGSM for the same model are not as large, and we have not an explanation for that.

The oscillations in $\omega$ on the axis of rotation near the surface are present also for the soft $N=1.5$ polytropes, while for $N=0.5$ they are larger. This indicates that terms in the field equations for $\omega$ are very sensitive to the presence of the surface and the accompanying Gibbs phenomenon. Even for $N=1.5$ polytropes, where the density goes to zero smoothly at the surface, there is a small scale Gibbs phenomenon, due to the finite number of grid points used to represent the region of integration.

\subsubsection{Method of interpolation}

An important factor for the local accuracy of models constructed with realistic equations of state is the method of interpolation of the energy vs. pressure data given in an 
Table 13. Detailed comparison of models with realistic EOSs (continued)

\begin{tabular}{|c|c|c|c|c|c|c|}
\hline Model & KEH(OR) & $\mathrm{KEH}(\mathrm{SF})$ & BGSM & diff1 & diff2 & diff3 \\
\hline Model & $\mathrm{L}(\mathrm{H}) \mathrm{mr}$ & & & & & \\
\hline$\varepsilon_{\mathrm{c}}\left[\mathrm{g} \mathrm{cm}^{-3}\right]$ & $1.2432200 \mathrm{e}+15$ & $1.2022600 \mathrm{e}+15$ & $1.2020680 \mathrm{e}+15$ & $-3 e-2$ & & $2 e-4$ \\
\hline$r_{\mathrm{p}} / r_{\mathrm{e}}$ & $5.5521963 \mathrm{e}-01$ & $5.5300000 \mathrm{e}-01$ & $5.5423989 \mathrm{e}-01$ & $-2 \mathrm{e}-3$ & $4 e-3$ & $-2 \mathrm{e}-3$ \\
\hline$\Omega\left[10^{4} \mathrm{~s}^{-1}\right]$ & $8.2083986 \mathrm{e}-01$ & $8.1441391 \mathrm{e}-01$ & $8.1328587 \mathrm{e}-01$ & $-9 e-3$ & $8 e-3$ & $1 e-3$ \\
\hline$M\left[M_{\odot}\right]$ & $3.3030613 \mathrm{e}+00$ & $3.2951034 \mathrm{e}+$ & $3.2897723 \mathrm{e}+00$ & $-4 e-3$ & $2 \mathrm{e}-3$ & $2 \mathrm{e}-3$ \\
\hline$M_{0}\left[M_{\odot}\right]$ & $3.8763017 \mathrm{e}+00$ & $3.8823439 \mathrm{e}+$ & $3.8749019 \mathrm{e}+00$ & $-4 e-4$ & $-2 \mathrm{e}-3$ & $2 \mathrm{e}-3$ \\
\hline$R_{\text {circ }}[\mathrm{km}]$ & $1.8375548 \mathrm{e}+01$ & $1.8488275 \mathrm{e}+01$ & $1.8475487 \mathrm{e}+01$ & $5 e-3$ & $-6 e-3$ & $7 e-4$ \\
\hline$c J / G M_{\odot}^{2}$ & $7.6167631 \mathrm{e}+00$ & $7.6420920 \mathrm{e}+00$ & $7.6199254 \mathrm{e}+00$ & $4 e-4$ & $-3 e-3$ & $3 e-3$ \\
\hline$I\left[10^{45} \mathrm{~g} \mathrm{~cm}^{2}\right]$ & $8.1547703 \mathrm{e}+00$ & $8.2466926 \mathrm{e}+00$ & $8.2341775 \mathrm{e}+00$ & $1 e-2$ & $-1 e-2$ & $2 \mathrm{e}-3$ \\
\hline$T / W$ & $2 \mathrm{e}-01$ & & 01 & $-1 e-2$ & $1 \mathrm{e}-2$ & $-4 e-4$ \\
\hline$Z_{\mathrm{p}}$ & $8.1941726 \mathrm{e}-01$ & $8.2094860 \mathrm{e}-01$ & $8.1829405 \mathrm{e}-$ & $-1 \mathrm{e}-3$ & $-2 e-3$ & $3 e-3$ \\
\hline$Z_{\mathrm{eq}}^{\mathrm{f}}$ & $-3.4820132 \mathrm{e}-01$ & $-3.4697590 \mathrm{e}-01$ & $-3.4552738 \mathrm{e}-01$ & $-8 \mathrm{e}-3$ & $4 e-3$ & $4 e-3$ \\
\hline$Z_{\mathrm{eq}}^{\mathrm{b}}$ & $2.4176003 \mathrm{e}+00$ & 2.42088 & $2.4115282 \mathrm{e}+00$ & $-3 e-3$ & $-1 \mathrm{e}-3$ & $4 e-3$ \\
\hline $\mathrm{e}$ & $e-01$ & & & $6 e-3$ & $-8 \mathrm{e}-3$ & $2 \mathrm{e}-3$ \\
\hline GRV2 & -2.2119 & 3.06701 & & & & \\
\hline GRV3 & -5.3441 & 7.80 & & & & \\
\hline Model & S)sr & & & & & \\
\hline$\varepsilon_{\mathrm{c}}\left[\mathrm{g} \mathrm{cm}^{-3}\right]$ & $e+15$ & & & & & \\
\hline$r_{\mathrm{p}} / r_{\mathrm{e}}$ & 1.0 & & & & & \\
\hline$M\left[M_{\odot}\right]$ & 1.41398 & 1.40970 & & $-3 e-3$ & & $-2 \mathrm{e}-4$ \\
\hline$M_{0}\left[M_{\odot}\right]$ & & & & $-1 e-3$ & $1 e-3$ & $-3 e-4$ \\
\hline$R_{\text {circ }}[\mathrm{km}]$ & & & & $-8 \mathrm{e}-5$ & $1 \mathrm{e}-4$ & $-4 \mathrm{e}-5$ \\
\hline$Z_{\mathrm{p}}$ & $6 e-01$ & 2.71062 & $114022 \mathrm{e}-01$ & $9 e-3$ & $-9 \mathrm{e}-3$ & $-3 e-4$ \\
\hline$Z_{\mathrm{eq}}^{\mathrm{f}}$ & $2.6889160 \mathrm{e}-01$ & $2.71062^{\prime}$ & $2.7114022 \mathrm{e}-01$ & $8 e-3$ & $-8 \mathrm{e}-3$ & $-3 e-4$ \\
\hline$Z_{\mathrm{eq}}^{\mathrm{b}}$ & $2.6889160 \mathrm{e}-01$ & 2.71062 & $2.7114022 \mathrm{e}-01$ & $8 e-3$ & $-8 \mathrm{e}-3$ & $-3 e-4$ \\
\hline GRV2 & -9.5 & & & & & \\
\hline GRV3 & & & & & & \\
\hline Model & & & & & & \\
\hline$\varepsilon_{\mathrm{c}}\left[\mathrm{g} \mathrm{cm}^{-3}\right]$ & & & & & & \\
\hline$r_{\mathrm{p}} / r_{\mathrm{e}}$ & & -01 & & & & \\
\hline$\Omega\left[10^{4} \mathrm{~s}^{-1}\right]$ & $2 \mathrm{e}-01$ & 7.00027 & & $-8 \mathrm{e}-4$ & $8 e-4$ & $-2 \mathrm{e}-5$ \\
\hline$M\left[M_{\odot}\right]$ & & & $51900 \mathrm{e}+00$ & $-1 e-3$ & $5 e-4$ & $5 e-4$ \\
\hline$M_{0}\left[M_{\odot}\right]$ & 1.54610 & 1.54668 & $58039 \mathrm{e}+00$ & $-2 e-4$ & $-4 e-4$ & $6 e-4$ \\
\hline$R_{\text {circ }}[\mathrm{km}]$ & & & $55153 \mathrm{e}+01$ & $1 e-4$ & $5 \mathrm{e}-5$ & $-2 e-4$ \\
\hline$c J / G M_{\odot}^{2}$ & $e+00$ & 1.31208 & $3 e+00$ & $-3 e-4$ & $-6 e-4$ & $9 \mathrm{e}-4$ \\
\hline$I\left[10^{45} \mathrm{~g} \mathrm{~cm}^{2}\right]$ & & & & & $-1 e-3$ & $9 \mathrm{e}-4$ \\
\hline$T / W$ & $e-01$ & & $6 e-01$ & $-6 e-3$ & $6 e-3$ & $-1 e-4$ \\
\hline$Z_{\mathrm{p}}$ & $2.8235936 \mathrm{e}-01$ & $2.8351010 \mathrm{e}-01$ & $2.8339205 \mathrm{e}-01$ & $4 e-3$ & $-4 \mathrm{e}-3$ & $4 e-4$ \\
\hline$Z_{\mathrm{eq}}^{\mathrm{f}}$ & $-2.1053187 \mathrm{e}-01$ & $-2.0979686 \mathrm{e}-01$ & $-2.0993514 \mathrm{e}-01$ & $-3 \mathrm{e}-3$ & $4 e-3$ & $-7 e-4$ \\
\hline$Z_{\mathrm{eq}}^{\mathrm{b}}$ & & & & & & $2 \mathrm{e}-4$ \\
\hline $\mathrm{e}$ & $9 e-01$ & $7.1756280 \mathrm{e}-01$ & $7.1517800 \mathrm{e}-01$ & $3 e-3$ & $-6 e-3$ & $3 e-3$ \\
\hline GRV2 & & $2.6460925 \mathrm{e}-02$ & $-2.3145939 \mathrm{e}-05$ & & & \\
\hline GRV3 & $2.1411844 \mathrm{e}-03$ & $3.8377908 \mathrm{e}-04$ & $-3.7280705 \mathrm{e}-05$ & & & \\
\hline
\end{tabular}

EOS table. While global quantities are not affected significantly, the virial identities for realistic EOSs, are sensitive to the interpolation scheme This can be considered to reflect the nature of the interpolation scheme as mentioned before. If we define the enthalpy $(H)$ by

$H \equiv \ln \left(\frac{\varepsilon+p}{\rho c^{2}}\right)$

the Gibbs-Duhem relation, which follows directly from the first law of thermodynamics, implies

$\frac{\mathrm{d} p}{\mathrm{~d} H}=\varepsilon+p$.
In the cubic Hermite interpolation, the Gibbs-Duhem relation is used to replace by $\nabla H$ the term $\nabla p /(\varepsilon+p)$ which appears in the hydrostationary equilibrium equation. If the tabulated function $p(H)$ fails to satisfy the above relation, then the hydrostationary equilibrium equation, which is derived from the Bianchi identity, is only approximately verified by the numerical solution, which results in increased error in the GRV2 and GRV3 virial identities.

The four point Lagrange interpolation does not satisfy the Gibbs-Duhem relation because it only reproduces the values of the discrete points, but there is no guarantee for 
Table 14. Detailed comparison of models with realistic EOSs (continued)

\begin{tabular}{ccccccc}
\hline Model & KEH(OR) & KEH(SF) & BGSM & diff1 & diff2 & diff3 \\
\hline Model & CLESmr & & & & & \\
$\varepsilon_{\mathrm{c}}\left[\mathrm{g} \mathrm{cm}^{-3}\right]$ & $4.2000000 \mathrm{e}+14$ & $4.2000000 \mathrm{e}+14$ & $4.1928573 \mathrm{e}+14$ & & & \\
$r_{\mathrm{p}} / r_{\mathrm{e}}$ & $5.3085938 \mathrm{e}-01$ & $5.3085938 \mathrm{e}-01$ & $5.3150938 \mathrm{e}-01$ & & & \\
$\Omega\left[10^{4} \mathrm{~s}^{-1}\right]$ & $6.0622590 \mathrm{e}-01$ & $6.0321091 \mathrm{e}-01$ & $6.0275394 \mathrm{e}-01$ & $-6 \mathrm{e}-3$ & $5 \mathrm{e}-3$ & $8 \mathrm{e}-4$ \\
$M\left[M_{\odot}\right]$ & $6.7113216 \mathrm{e}+00$ & $6.6627293 \mathrm{e}+00$ & $6.6394450 \mathrm{e}+00$ & $-1 \mathrm{e}-2$ & $7 \mathrm{e}-3$ & $4 \mathrm{e}-3$ \\
$M_{0}\left[M_{\odot}\right]$ & $8.3688660 \mathrm{e}+00$ & $8.3951155 \mathrm{e}+00$ & $8.3562334 \mathrm{e}+00$ & $-2 \mathrm{e}-3$ & $-3 \mathrm{e}-3$ & $5 \mathrm{e}-3$ \\
$R_{\text {circ }}[\mathrm{km}]$ & $2.8623928 \mathrm{e}+01$ & $2.8660015 \mathrm{e}+01$ & $2.8649929 \mathrm{e}+01$ & $9 \mathrm{e}-4$ & $-1 \mathrm{e}-3$ & $3 \mathrm{e}-4$ \\
$c J / G M_{\odot}^{2}$ & $3.6211896 \mathrm{e}+01$ & $3.6395860 \mathrm{e}+01$ & $3.6124793 \mathrm{e}+01$ & $-2 \mathrm{e}-3$ & $-5 \mathrm{e}-3$ & $8 \mathrm{e}-3$ \\
$I\left[0^{45} \mathrm{gm}^{2}\right]$ & $5.2494829 \mathrm{e}+01$ & $5.3026814 \mathrm{e}+01$ & $5.2671785 \mathrm{e}+01$ & $3 \mathrm{e}-3$ & $-1 \mathrm{e}-2$ & $7 \mathrm{e}-3$ \\
$T / W$ & $1.9699723 \mathrm{e}-01$ & $1.9169339 \mathrm{e}-01$ & $1.9176893 \mathrm{e}-01$ & $-3 \mathrm{e}-2$ & $3 \mathrm{e}-2$ & $-4 \mathrm{e}-4$ \\
$Z_{\mathrm{p}}$ & $1.4566262 \mathrm{e}+00$ & $1.4908621 \mathrm{e}+00$ & $1.4820382 \mathrm{e}+00$ & $2 \mathrm{e}-2$ & $-2 \mathrm{e}-2$ & $6 \mathrm{e}-3$ \\
$Z_{\mathrm{eq}}^{\mathrm{f}}$ & $-4.0695375 \mathrm{e}-01$ & $-4.0247884 \mathrm{e}-01$ & $-4.0233644 \mathrm{e}-01$ & $-1 \mathrm{e}-2$ & $1 \mathrm{e}-2$ & $4 \mathrm{e}-4$ \\
$Z_{\mathrm{eq}}^{\mathrm{b}}$ & $5.8899056 \mathrm{e}+00$ & $6.1402011 \mathrm{e}+00$ & $6.0575089 \mathrm{e}+00$ & $3 \mathrm{e}-2$ & $-4 \mathrm{e}-2$ & $1 \mathrm{e}-2$ \\
$\mathrm{e}$ & $7.1993250 \mathrm{e}-01$ & $7.2912408 \mathrm{e}-01$ & $7.2745777 \mathrm{e}-01$ & $1 \mathrm{e}-2$ & $-1 \mathrm{e}-2$ & $2 \mathrm{e}-3$ \\
GRV2 & $-7.4679128 \mathrm{e}-03$ & $7.7678310 \mathrm{e}-02$ & $5.8321139 \mathrm{e}-04$ & & & \\
GRV3 & $-1.5019570 \mathrm{e}-01$ & $9.3185944 \mathrm{e}-04$ & $8.1316752 \mathrm{e}-04$ & & & \\
\hline
\end{tabular}

the reproduction of the derivatives. This explains why the GRV2 and GRV3 errors are bad, even in the nonrotating case (GRV2 = 3E-03, GRV3 = 1E-02 for model Lsr) as compared to GRV2 $\sim 10^{-14}$ for polytropic models (see e.g. Bonazzola et al. 1993). The GRV2 and GRV3 error indicators thus do not reflect the precision of the code but the bad thermodynamical behavior of the tabulated EOS.

The advantage of the cubic Hermite interpolation is that the Gibbs-Duhem relation is automatically satisfied because this interpolation reproduces not only the values themselves but also the derivatives (Swesty 1996). Moreover, in our case, the energy density and the baryon number density are obtained by

$\varepsilon=\frac{p}{H} \frac{\mathrm{d} \log p}{\mathrm{~d} \log H}-p$,

$n=\frac{\varepsilon+p}{m_{\mathrm{B}} c^{2}} \exp (-H)$.

Because of these equations, the Gibbs-Duhem relation is satisfied in every point. Note also that the value of $\varepsilon$ obtained in this way coincides exactly with $\varepsilon_{i}$ at the points in the tabulated equation of state.

\subsection{Conclusion}

The comparison of three different codes for constructing rapidly rotating relativistic neutron star models demonstrates that the BGSM and KEH schemes used are highly accurate for typical polytropic models - when the field equations are solved to infinity - and for models constructed with realistic equations of state, that do not have phase transitions. If one approximates neutron stars as constant density stars, then Gibbs phenomena at the discontinuous surface reduce the accuracy of the computed models. If high accuracy in such models and in models with phase transitions is desired, then modified numerical schemes - free of Gibbs phenomena - need to be used.
Such numerical schemes could employ, for example, surface fitted coordinates. Such a scheme has been presented recently by Bonazzola et al. (1998a) in the framework of spectral methods and looks promising for rotating stellar models. Further, we demonstrated that the metric potentials are subject to various local oscillatory behaviors, even if integrated quantities have very good accuracy. This observation is important for the effort of constructing initial data for the numerical evolution of rotating relativistic neutron star models.

Acknowledgements. We would like to thank S. Bonazzola and J.L. Friedman for very helpful discussions. This research has been supported in part by NSF grant PHY-9507740 and by the Grant-in-Aid for Scientific Research of the Japanese Ministry of Education, Science and Culture. N.S. wishes to acknowledge the generous hospitality of the Max Planck Institute for Gravitational Physics, Potsdam, where part of this paper was completed.

\section{References}

Arnett W.D., Bowers R.L., 1977, ApJS 33, 415

Baym G., Bethe H.A., Pethick C.J., 1971, Nucl. Phys. A, 175, 225

Baym G., Pethick C.J., Sutherland P., 1971, ApJ 170, 299

Bethe H.A., Johnson M., 1974, Nucl. Phys. A 230, 1

Bonazzola S., 1973, ApJ 182, 335

Bonazzola S., Frieben J., Gourgoulhon E., Marck J.A., 1996, in Proc. Third International Conference on Spectral and High Order Methods (ICOSAHOM'95), Ilin A.V. \& Scott L.R. (eds.). Houston Journal of Mathematics, Houston

Bonazzola S., Gourgoulhon E., 1994, Class. Quantum. Grav. 11,1775

Bonazzola S., Gourgoulhon E., Marck J.A., 1997, in Relativistic Gravitation and Gravitational Radiation, Marck J.-A. \& Lasota J.-P. (eds.). Cambridge University Press, Cambridge 


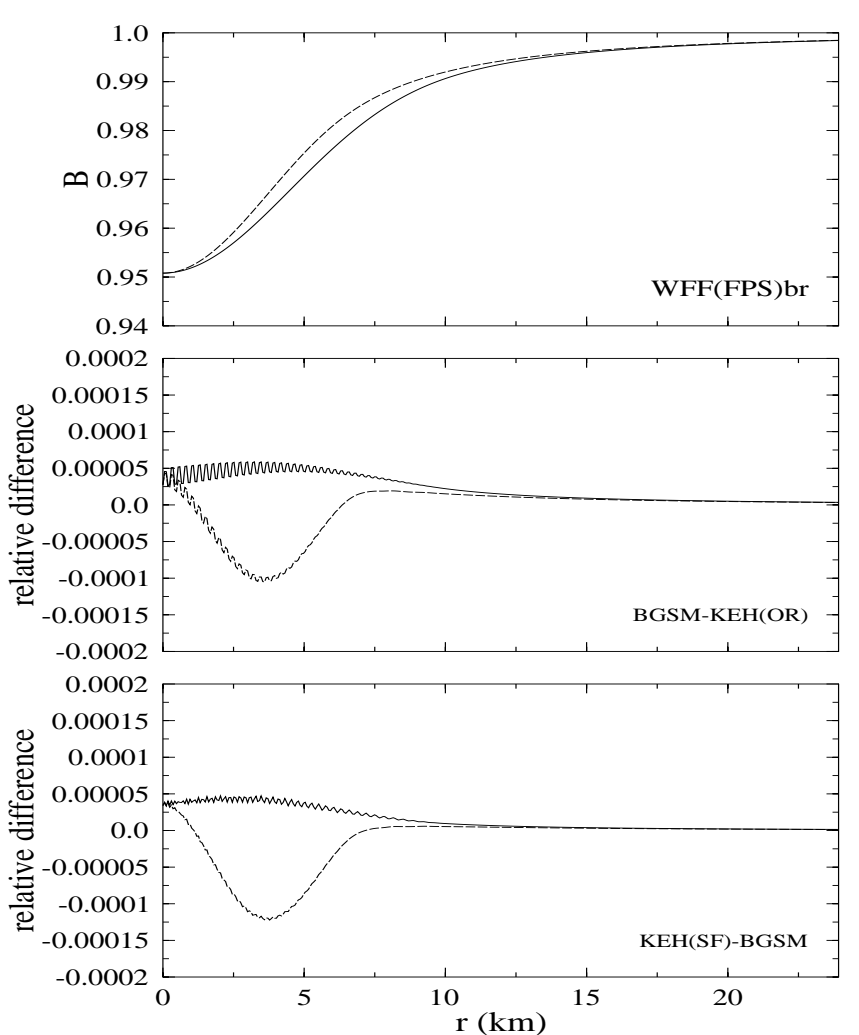

Fig. 2. Same as Fig. 1 but for the metric potential $B$ of model WFF(FPS) $b r$

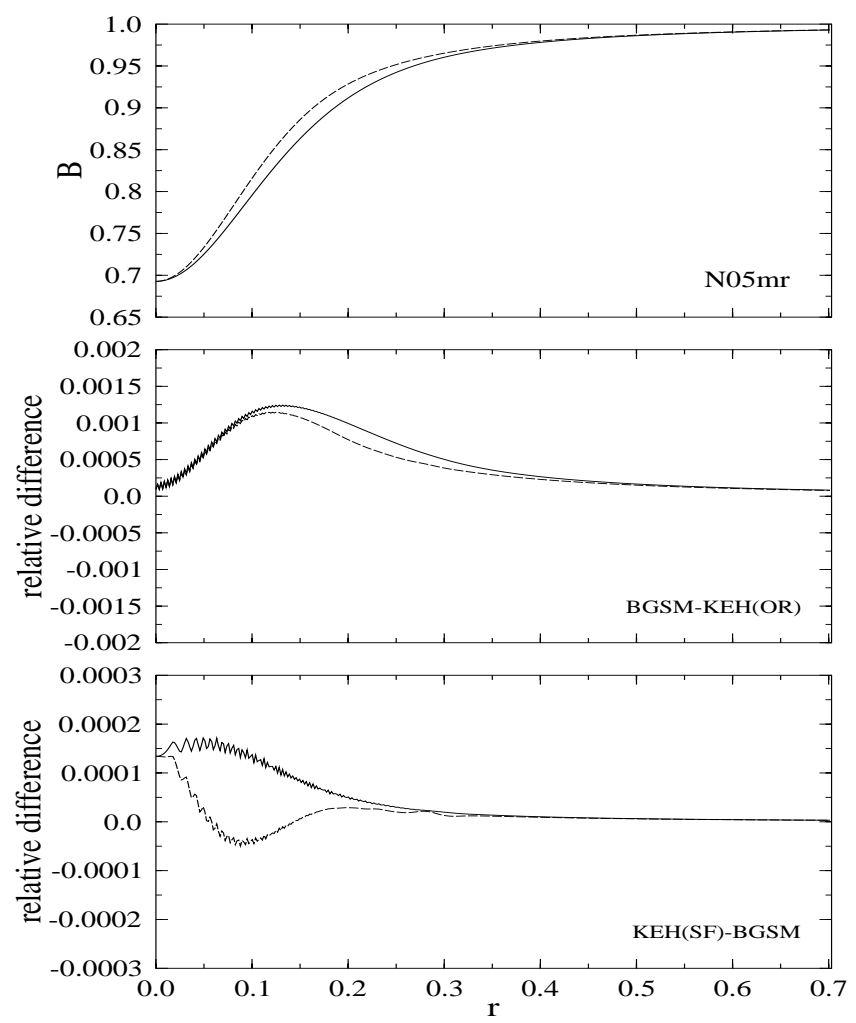

Fig. 3. Same as Fig. 1 but for the metric potential $B$ of model N05mr

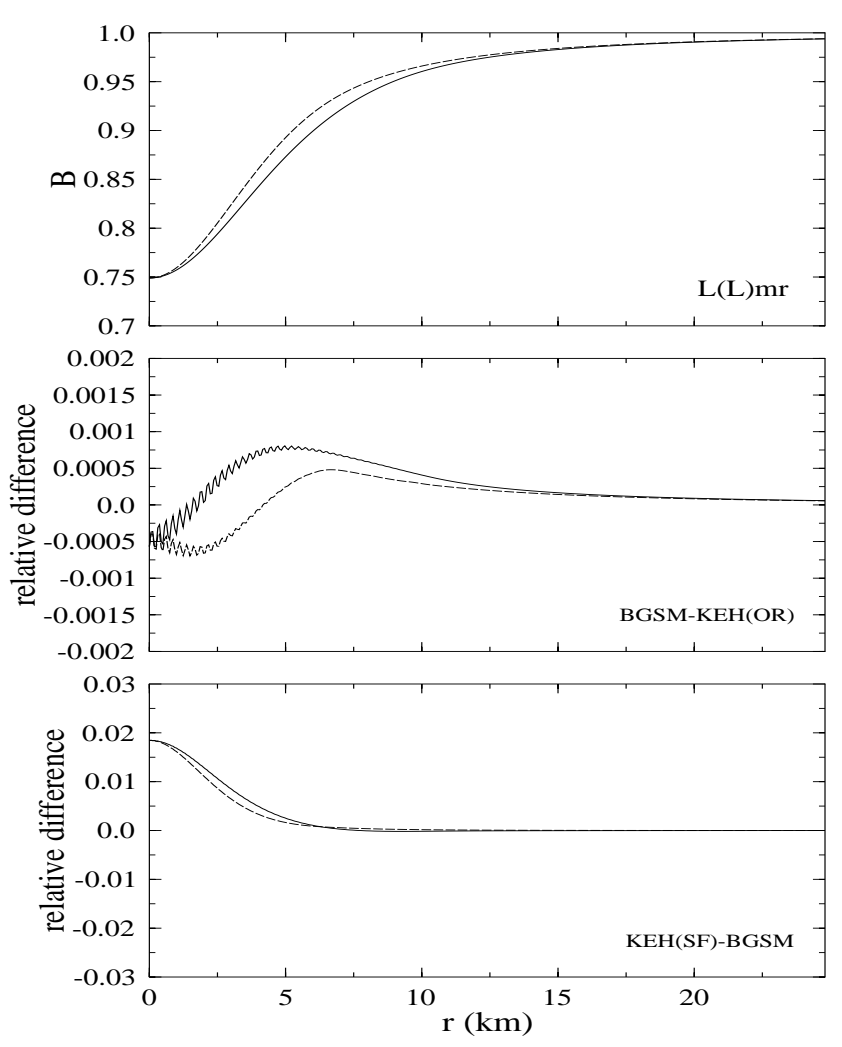

Fig. 4. Same as Fig. 1 but for the metric potential $B$ of model $\mathrm{L}(\mathrm{L}) m r$
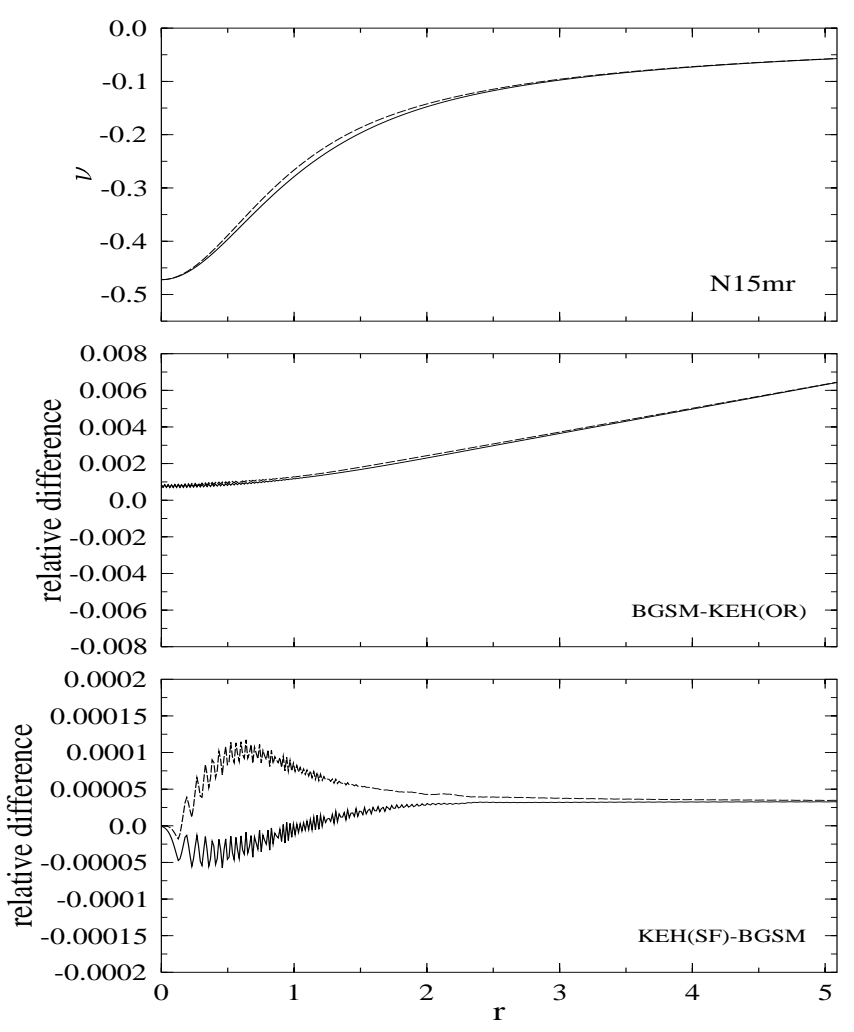

Fig. 5. Same as Fig. 1 but for the metric potential $\nu$ of model N15mr 

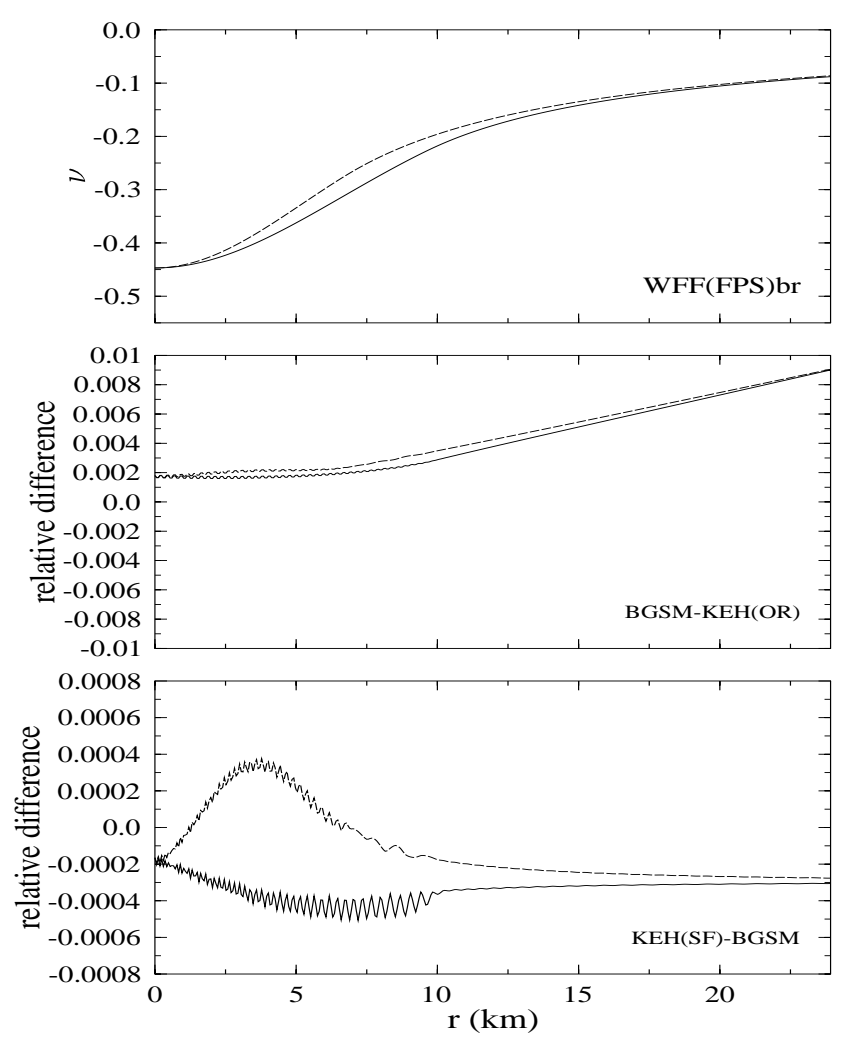

Fig. 6. Same as Fig. 1 but for the metric potential $\nu$ of model $\mathrm{WFF}(\mathrm{FPS}) b r$
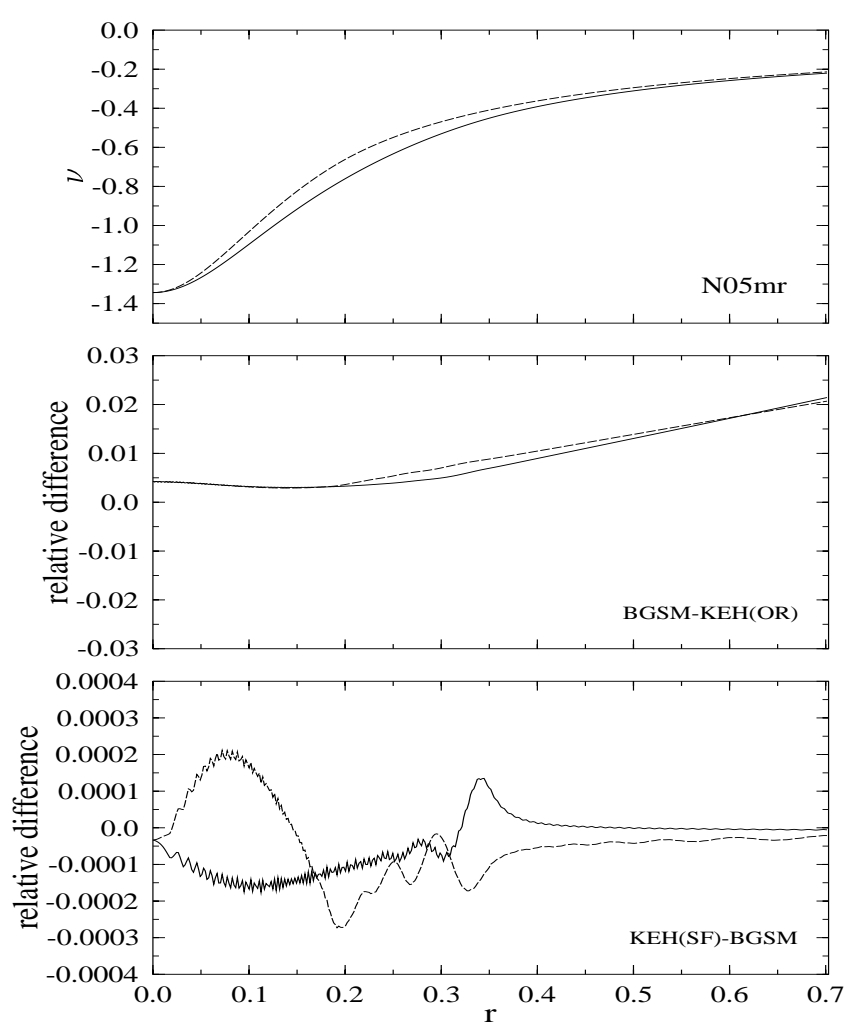

Fig. 7. Same as Fig. 1 but for the metric potential $\nu$ of model N05mr
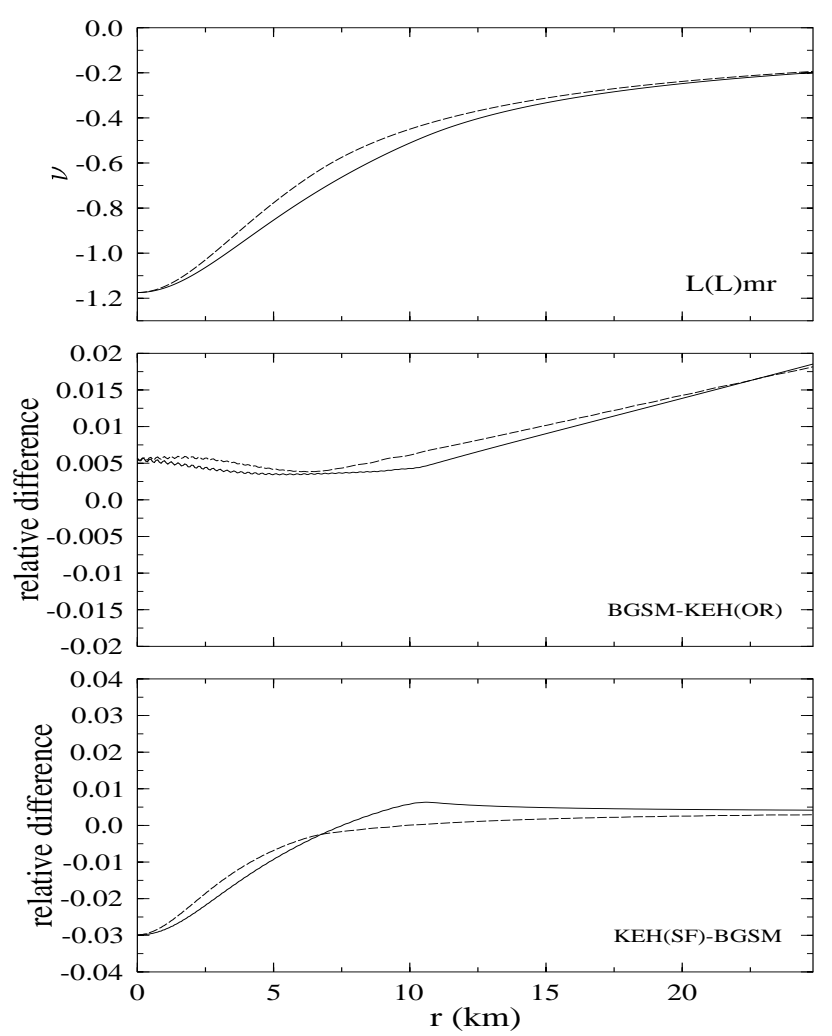

Fig. 8. Same as Fig. 1 but for the metric potential $\nu$ of model $\mathrm{L}(\mathrm{L}) m r$

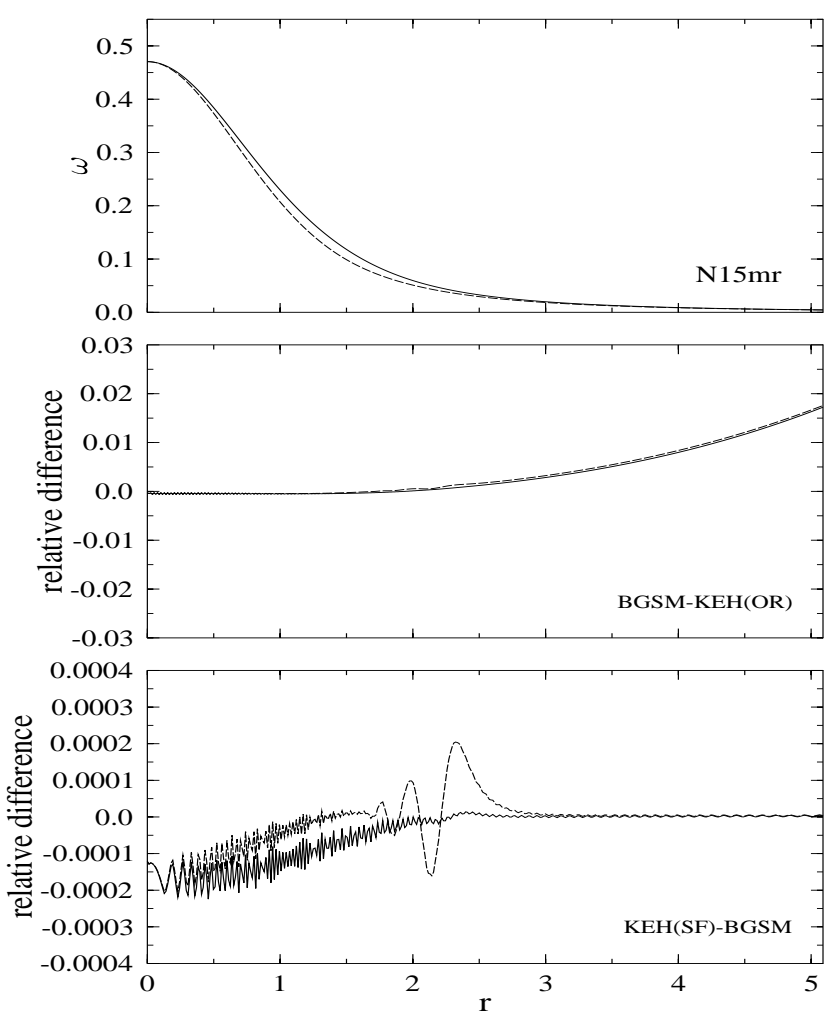

Fig. 9. Same as Fig. 1 but for the metric potential $\omega$ (in units of $\Omega$ ) of model N15mr 


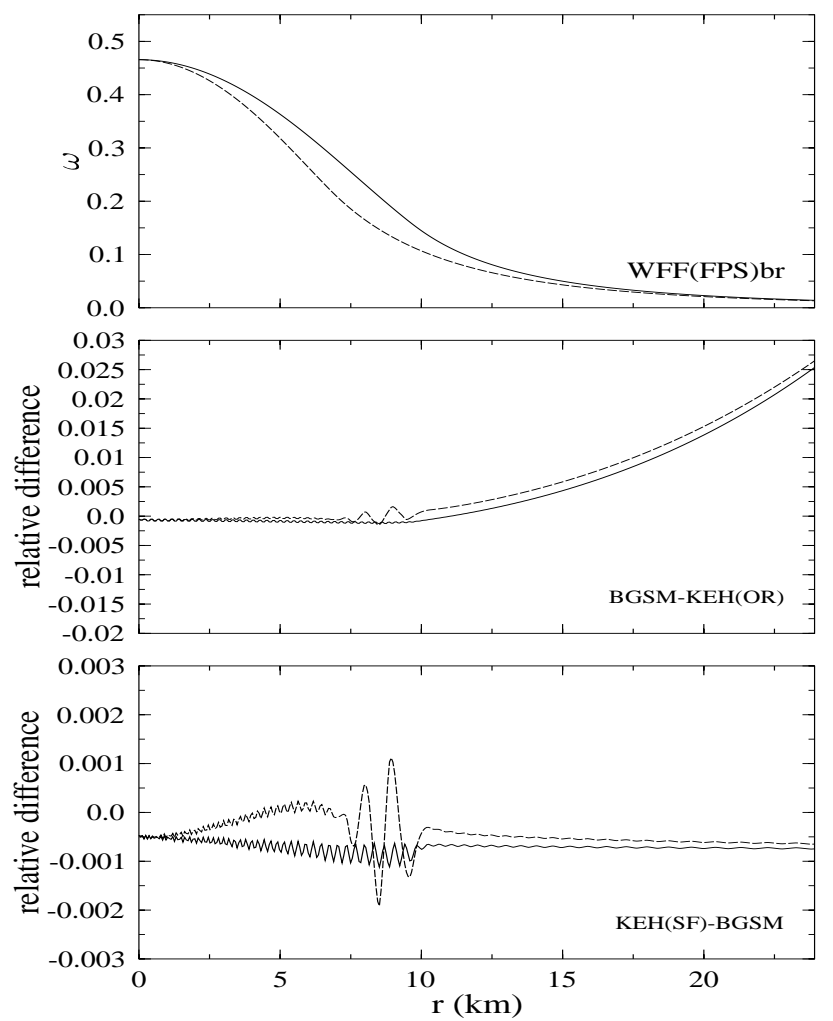

Fig. 10. Same as Fig. 1 but for the metric potential $\omega$ (in units of $\Omega$ ) of model WFF(FPS) $b r$
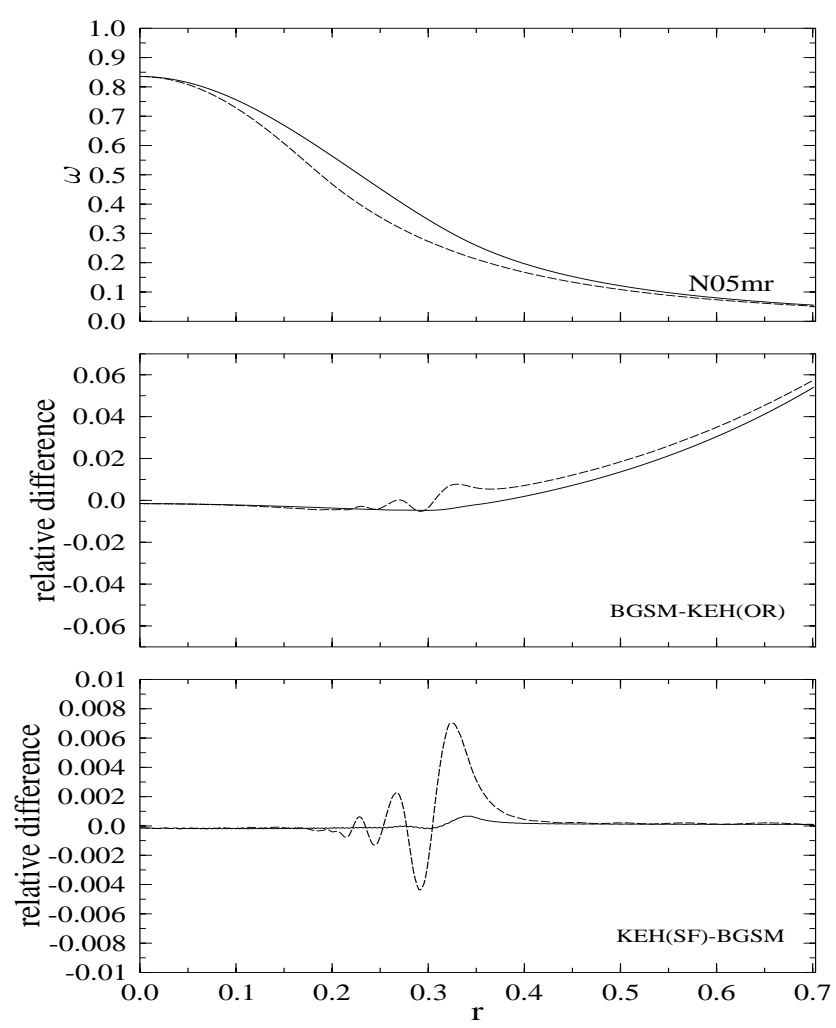

Fig. 11. Same as Fig. 1 but for the metric potential $\omega$ (in units of $\Omega$ ) of model N05mr

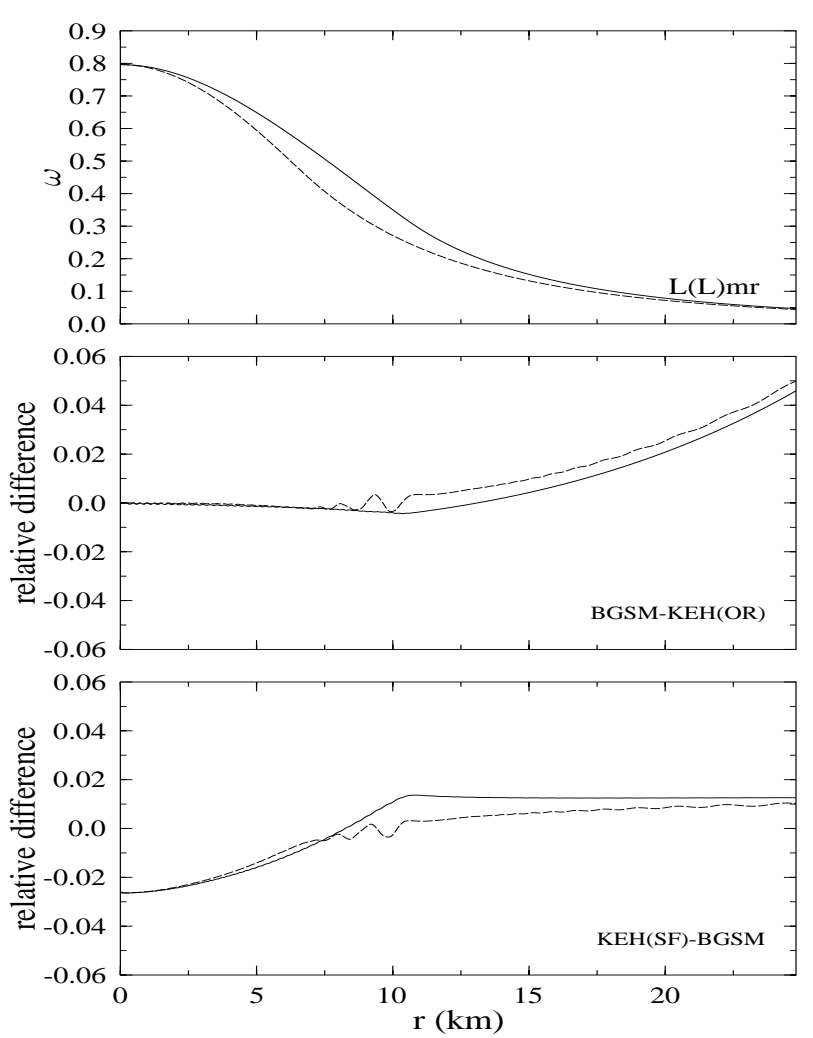

Fig. 12. Same as Fig. 1 but for the metric potential $\omega$ (in units of $\Omega$ ) of model L(L) $m r$
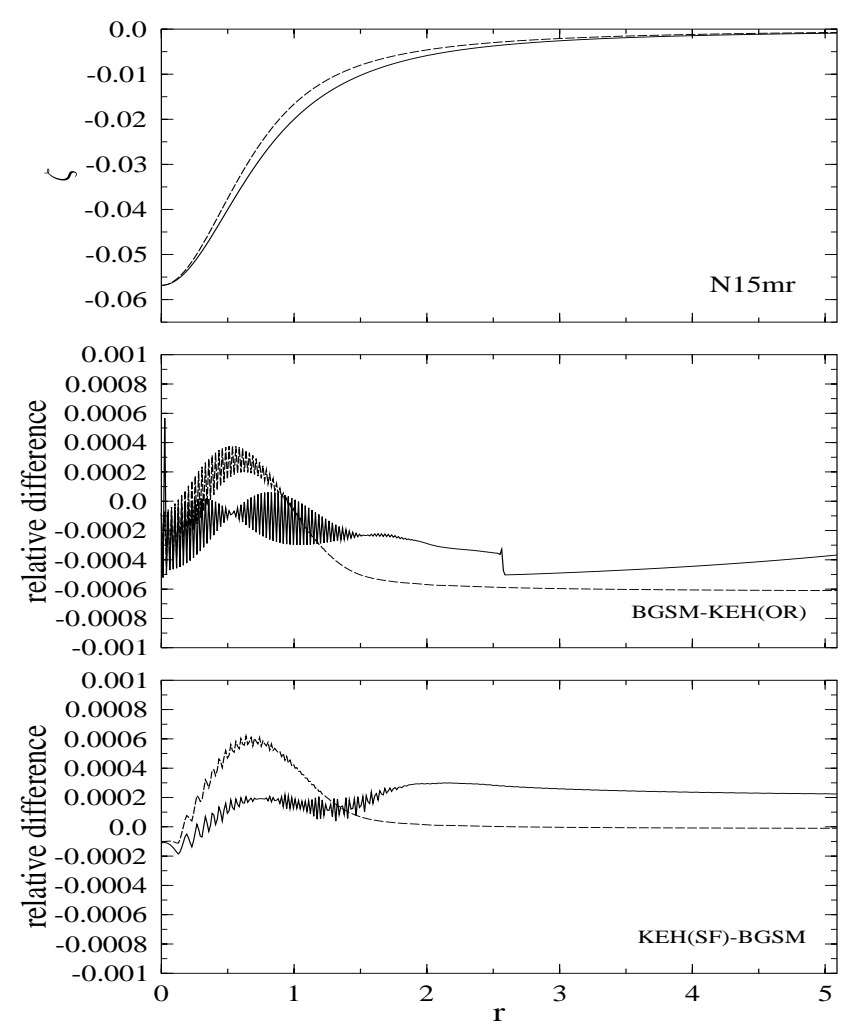

Fig. 13. Same as Fig. 1 but for the metric potential $\zeta$ of model N15mr 


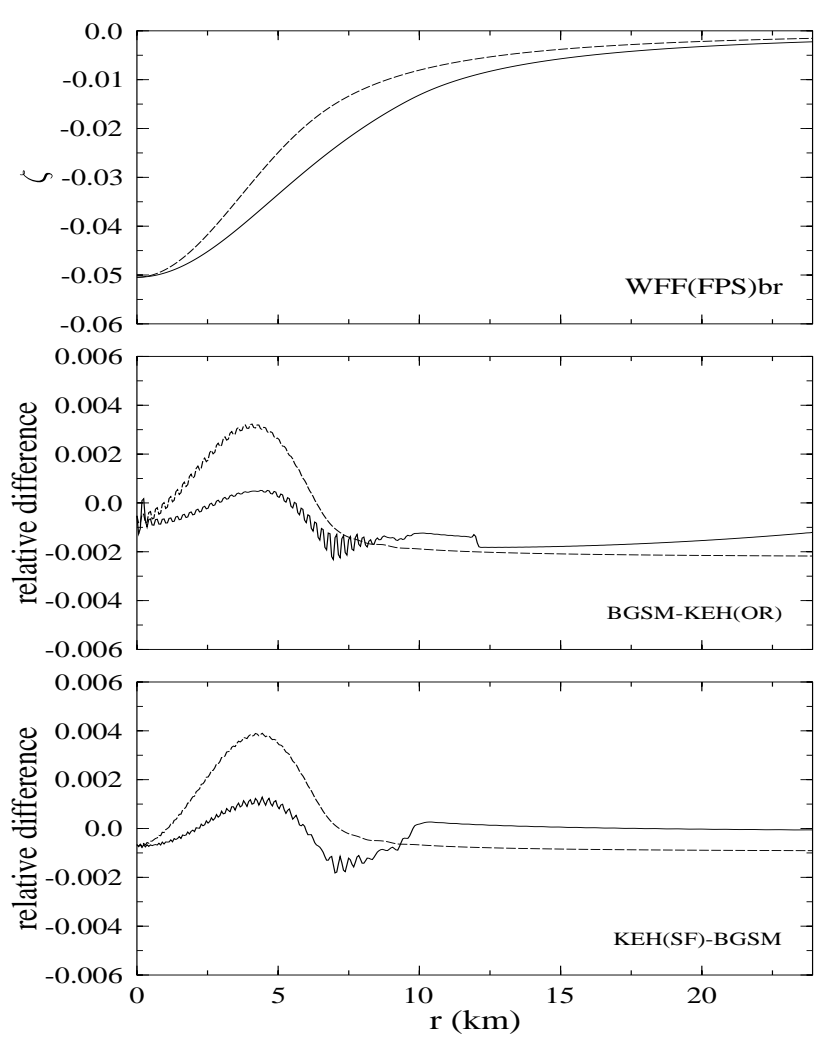

Fig. 14. Same as Fig. 1 but for the metric potential $\zeta$ of model WFF(FPS) $b r$

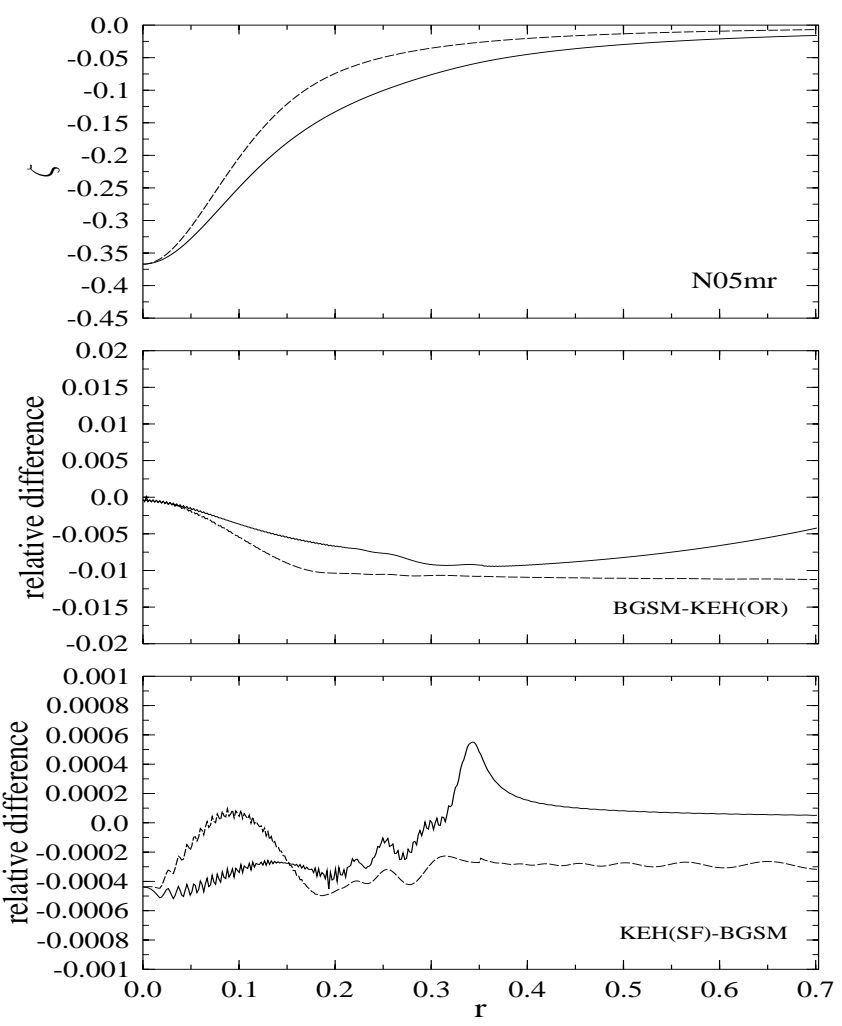

Fig. 15. Same as Fig. 1 but for the metric potential $\zeta$ of model N05mr
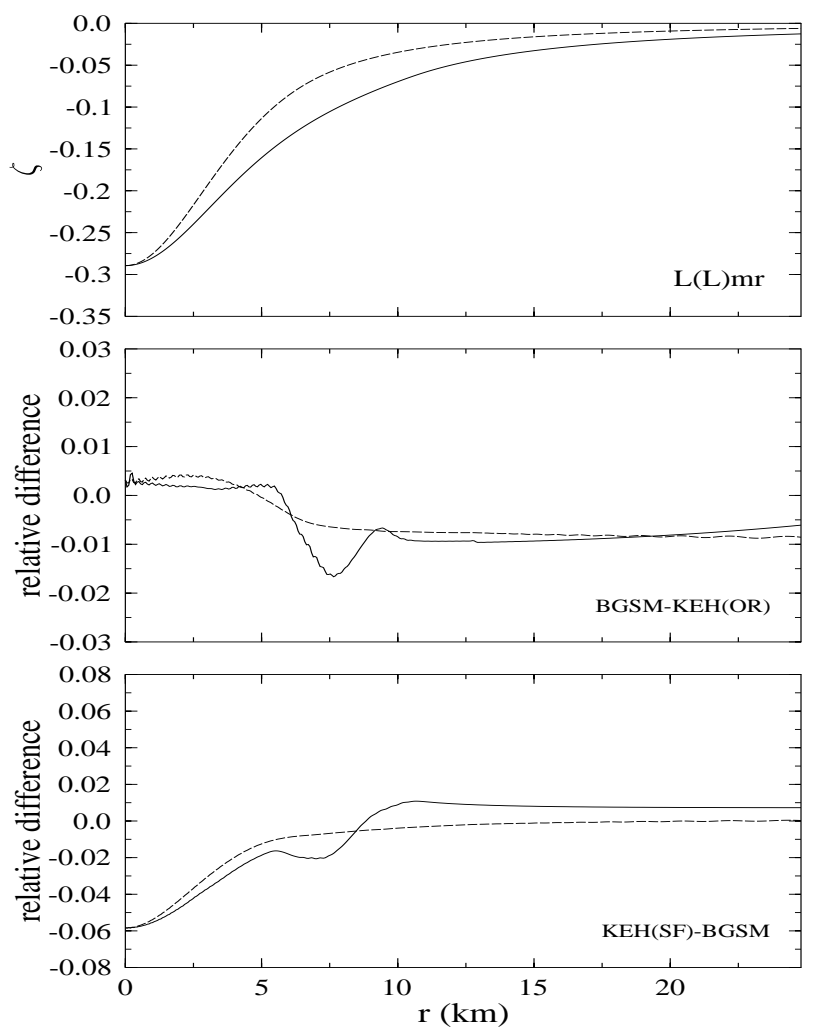

Fig. 16. Same as Fig. 1 but for the metric potential $\zeta$ of model $\mathrm{L}(\mathrm{L}) m r$

Bonazzola S., Gourgoulhon E., Marck J.A., 1998a, Numerical approach for high precision 3-D relativistic star models, Phys. Rev. D (in press) preprint astro-ph/9803086

Bonazzola S., Gourgoulhon E., Marck J.A., 1998b, Spectral methods in relativistic astrophysics, to appear in J. Comp. Appl. Math.

Bonazzola S., Gourgoulhon E., Salgado M., Marck J.A., 1993, A\&A 278, 421

Bonazzola S., Schneider J., 1974, ApJ 191, 273

Butterworth E.M., 1976, ApJ 204, 561

Butterworth E.M., Ipser J.R., 1975, ApJL 200, L103

Butterworth E.M., Ipser J.R., 1976, ApJ 204, 200

Canuto V., Chitre S.M., 1974, Phys. Rev. D 9, 1587

Carter B., 1969, J. Math. Phys. 10, 70

Cook G.B., Shapiro S.L., Teukolsky S.A., 1992, ApJ 398, 203

Cook G.B., Shapiro S.L., Teukolsky S.A., 1994a, ApJ 422, 273

Cook G.B., Shapiro S.L., Teukolsky S.A., 1994b, ApJ 424, 823

Eriguchi Y., Hachisu I., Nomoto K., 1994, MNRAS 266, 179

Feynman R.P., Metropolis N., Teller E., 1949, Phys. Rev. 75, 1561

Friedman J.L., Imamura J.N., Durisen R.H., Parker L., 1988, Nat 336, 560

Friedman J.L., Ipser J.R., Parker L., 1984, Nat 312, 255

Friedman J.L., Ipser J.R., Parker L., 1986, ApJ 304, 115

Friedman J.L., Ipser J.R., Parker L., 1989, Phys. Rev. Lett. 62,3015

Friedman B., Pandharipande V.R., 1981, Nucl. Phys. A 361, 502

Gourgoulhon E., Bonazzola S., 1994, Class. Quantum. Grav. 11,443 
Gottlieb D., Orszag S., 1977, Numerical Analysis of Spectral Methods: Theory and Application, Regional Conference Series in Applied Mathematics, Vol. 26 (Philadelphia: SIAM)

Hachisu I., 1986, ApJS 61, 479

Komatsu H., Eriguchi Hachisu I., 1989a, MNRAS 237, 355

Komatsu H., Eriguchi Hachisu I., 1989b, MNRAS 239, 153

Lorenz C.P., Ravenhall D.G., Pethick C.J., 1993, Phys. Rev. Lett. 70,379

Negele J.W., Vautherin D., 1973, Nucl. Phys. A 207, 298

Ostriker J.P., Mark J.W.-K., 1968, ApJ 151, 1075

Pandharipande V.R., 1971, Nucl. Phys. A 178, 123

Pandharipande V.R., Pines D., Smith R.A., 1976, ApJ 208, 550

Pandharipande V.R., Smith R.A., 1975, Phys. Lett. 59B, 15

Papapetrou A., 1966, Ann. Inst. H. Poincaré A4, 83

Salgado M., Bonazzola S., Gourgoulhon E., Haensel P., 1994a, A\&A 291, 155

Salgado M., Bonazzola S., Gourgoulhon E., Haensel P., 1994b,
A\&AS 108, 455

Stergioulas N., Friedman J.L., 1995, ApJ 444, 306

Stergioulas N., 1998, "Rotating Stars in Relativity", Living Reviews in Relativity, 1, 1998-8, http:// www.livingreviews.org

Swesty F.D., 1996, J. Comp. Phys. 127, 118

Tassoul J.L., 1978, Theory of Rotating Stars. Princeton Univ. Press: Princeton, NJ

Taylor J.H., Weisberg J.M., 1989, ApJ 345, 434

Tooper R.F., 1965, ApJ 142, 1541

Thorsett S.E., Arzoumanian Z., McKinnon M.M., Taylor J.H., 1993, ApJ 405, L29

van Kerkwijk M.H., van Paradijs J., Zuiderwijk E.J., 1995, A\&A 303, 497

Wiringa R.B., Fiks V., Farbrocini A., 1988, Phys. Rev. C 38, 1010

Wolszczan A., 1997, in Relativistic Gravitation and Gravitational Radiation, Marck J.-A. \& Lasota J.-P. (eds.). Cambridge University Press, Cambridge 